\title{
The Masses and Spins of Neutron Stars and Stellar-Mass Black Holes
}

\author{
M. Coleman Miller ${ }^{1}$ and Jon M. Miller ${ }^{2}$ \\ ${ }^{1}$ University of Maryland, Department of Astronomy and Joint Space-Science Institute, \\ College Park, MD 20742-2421 \\ ${ }^{2}$ University of Michigan, Department of Astronomy, 500 Church Street, Ann Arbor, MI \\ 48109-1042
}

\begin{abstract}
Stellar-mass black holes and neutron stars represent extremes in gravity, density, and magnetic fields. They therefore serve as key objects in the study of multiple frontiers of physics. In addition, their origin (mainly in core-collapse supernovae) and evolution (via accretion or, for neutron stars, magnetic spindown and reconfiguration) touch upon multiple open issues in astrophysics.

In this review, we discuss current mass and spin measurements and their reliability for neutron stars and stellar-mass black holes, as well as the overall importance of spins and masses for compact object astrophysics. Current masses are obtained primarily through electromagnetic observations of binaries, although future microlensing observations promise to enhance our understanding substantially. The spins of neutron stars are straightforward to measure for pulsars, but the birth spins of neutron stars are more difficult to determine. In contrast, even the current spins of stellar-mass black holes are challenging to measure. As we discuss, major inroads have been made in black hole spin estimates via analysis of iron lines and continuum emission, with reasonable agreement when both types of estimate are possible for individual objects, and future X-ray polarization measurements may provide additional independent information. We conclude by exploring the exciting prospects for mass and spin measurements from future gravitational wave detections, which are expected to revolutionize our understanding of strong gravity and compact objects.
\end{abstract}

Keywords: accretion disks, black holes, gravitational waves, neutron stars 


\section{Contents}

1 Introduction 3

1.1 Why do we care? . . . . . . . . . . . . . 3

1.2 General theory of neutron stars and black holes . . . . . . 6

2 Masses 15

2.1 The maximum mass of a neutron star . . . . . . . . . . 15

2.2 The binary mass function . . . . . . . . . . . . 19

2.3 Binary pulsars and post-Keplerian parameters . . . . . . . 20

3 Spins 23

3.1 Neutron Star Spins . . . . . . . . . . . . . . . . . 23

3.1.1 Non-accreting pulsars . . . . . . . . . . . . . . 24

3.1.2 Accreting pulsars . . . . . . . . . . . . 27

3.2 Natal Neutron Star Spin . . . . . . . . . . . . . . . . . . . . 29

3.3 Measuring Black Hole Spin with the Disk . . . . . . . . . . . . 31

3.3.1 Geometrically thin, radiatively efficient disks with an

ISCO . . . . . . . . . . . . . . . . 31

3.3.2 Black hole spin axis orientation . . . . . . . . . . . 33

3.4 Relativistic Disk Reflection . . . . . . . . . . . . . . . . . . . . . . . . .

3.5 The Accretion Disk Continuum . . . . . . . . . . . . . . 40

3.6 Quasi-Periodic Brightness Oscillations . . . . . . . . . . 44

3.7 Spin from X-ray Polarization . . . . . . . . . . . . . . . . . . . . . . . . . 48

3.8 Theoretical Expectations for Spins . . . . . . . . . . . . . . 48

4 Gravitational Waves 49

4.1 Mass measurements from gravitational waves . . . . . . . . 52

4.2 The effects of spin . . . . . . . . . . . . . 54

5 Conclusions

6 Acknowledgements 59 


\section{Introduction}

Neutron stars were thought to be objects of mostly academic interest until the discovery of pulsars by Jocelyn Bell and Antony Hewish [1]. The acceptance of black holes as real sources was much more gradual. The decades since the first proposals that quasars and other AGN were powered by supermassive black holes [2, 3] and the discovery of Cyg X-1 [4, 5, 6, 7] and other stellar-mass black hole candidates have seen progressively more definitive identification of some objects as black holes. Now the discussion has turned from the reality of these objects to their properties and what they can tell us about fundamental physics and astrophysics.

In this review we will explore what has been learned about the masses and spins of neutron stars and stellar-mass black holes, and what insights the methods and results have yielded. We begin in $\S 1.1$ by giving context to these measurements, then present an overview of the general theory in $\$ 1.2$. We discuss how masses are measured in $\S 2$, how spins are measured in $\S 3$, and what we can anticipate from future gravitational wave measurements in $\S 4$. We present our summary in $\S 5$. This collection of topics has not previously appeared in a single review, but there are several excellent previous articles that discuss some of the individual subjects. For more detail about the masses and other properties of binary and millisecond pulsars we recommend the review by Lorimer [8]; for additional information about the masses of stellar-mass black holes we suggest the recent work by Kreidberg et al. [9]; for a summary of the physics of $\mathrm{Fe} \mathrm{K} \alpha$ lines a good reference is the review by Reynolds and Nowak [10]; for a discussion of the continuum fitting method of spin determinations we recommend the review by McClintock et al. [11]; and for more technical details about the physics and astrophysics of gravitational waves we suggest the reviews by Blanchet [12] and Sathyaprakash and Schutz [13].

\subsection{Why do we care?}

Fundamental physics.-Neutron star and black holes are excellent probes of strong gravity and dense matter. For example, although Einstein's theory of gravity, general relativity, has been confirmed in every experiment and observation yet performed, all such data are restricted to weak gravity, in the sense that $G M / r c^{2}$ (where $G$ and $c$ are respectively Newton's gravitational constant and the speed of light, $M$ is the gravitational mass of an object, and $r$ is size of the source, e.g., the separation between the two neutron stars 
in a binary) is less than $\sim 10^{-5}$ (e.g., Figure 1 in [14]). Thus the predictions of strong-field gravity with $G M / r c^{2} \sim 1$, such as the existence of unstable circular orbits, are not easily tested. Neutron stars, with $G M / R c^{2} \sim 0.1-0.3$, and black holes, with $G M / R c^{2} \sim 1$ at their event horizons, are thus natural laboratories for strong gravity.

Neutron stars are also invaluable objects for the study of cold dense matter. Their cores are believed to reach densities that are several times nuclear saturation density $\rho_{0} \sim 2.6 \times 10^{14} \mathrm{~g} \mathrm{~cm}^{-3}$ (the density of atomic nuclei on Earth), and their composition could be anything from mainly neutrons and protons to condensates or quark hybrid matter (see [15] for a recent review). The boundary in mass between neutron stars and black holes, and the radii of neutron stars, would provide key hints to the nature of this high-density matter [16].

Astrophysics.-As astrophysical sources, stellar-mass black holes provide prototypes for the supermassive black holes in active galactic nuclei (AGN), with the advantage that we receive much greater flux from stellar-mass black holes and their characteristic timescales are much shorter than in AGN. Thus the study of state changes and quasi-periodic variability in stellar-mass black holes can yield unique insights into AGN. Moreover, jets from accreting neutron stars and stellar-mass black holes can serve as probes of magnetohydrodynamics and plasma physics in relativistic conditions and as prototypes for the wide range of jets in the universe, from protostellar systems to supermassive black holes. These jets are believed to have a significant impact on their environment, especially on the evolution of galaxies and galaxy clusters and the formation of structure [17], so the relatively nearby neutron stars and stellar-mass black holes give us an invaluable close up opportunity to study jet phenomena.

As the remnants of core-collapse supernovae, neutron stars and black holes are also windows into the collapse and explosion processes. The further evolution of these systems in accreting binaries can tell us about the accretion process, e.g., the total amount of mass that is typically deposited on the compact object. As we discuss in $\$ 1.2$, black holes that are currently in a binary cannot have accreted enough mass from their companion to change their mass substantially. It is also difficult for a stellar-mass black hole to accrete enough mass to change significantly the magnitude of its angular momentum. Thus the current mass and spin magnitude of a stellar-mass black hole is a relatively faithful record of its birth mass and spin magnitude, which in turn tells us about the conditions in the pre-supernova star just 
before the collapse. In contrast, there is an ongoing debate about whether the spin direction of black holes can be altered substantially by accretion. As we will see, this has a significant impact on the reliability of some types of spin measurement.

In distinction to black holes, neutron stars can have their mass changed substantially by accretion from a companion, and the spin magnitudes and directions of accreting neutron stars are essentially wholly determined by the accretion in low-mass X-ray binaries, and probably also in high-mass X-ray binaries. It is not possible at this time to measure the masses of isolated neutron stars except perhaps by microlensing (and then the identification of the object as a neutron star is not certain). The current spins of pulsars are obviously straightforward to measure, but these spins have been affected strongly by magnetic spindown. However, as we discuss in $\S 3$, there are indirect ways to guess the birth spin of isolated pulsars based on the spindown energy injected into the surrounding supernova remnant, and such studies have somewhat surprisingly pointed towards relatively long birth periods (a few tenths of a second) being typical.

Neutron stars and black holes in dense stellar systems such as globular clusters and the Galactic center are also probes of the dynamics in such systems. For example, millisecond pulsars are thought to be spun up by accretion from a companion (and what limits their spin is another issue we will address). This can happen in an isolated binary, but millisecond pulsars are much more common per total stellar mass in dense stellar systems. This is thought to be because dynamical interactions, particularly binary-single encounters, can allow previously isolated neutron stars to swap into binaries and acquire companions that later donate mass and spin up the neutron star. As a result, one would expect that such stars are systematically more massive than neutron stars that have not accreted and spun up. There is, likewise, a debate about whether there should be a substantial number of stellar-mass black holes in globular clusters. Work for many years has found that the holes would sink into a dense subcluster and self-eject [18, 19, 20, 21, 22], but the recent discovery of black hole candidates in several globular clusters [23, 24, 25, 26, 27, 28] has led to re-examination of globular cluster black hole dynamics [29, 30, 31, 32]. It could be that black holes in globular clusters (where they have billions of years to eat other stars) or low-metallicity environments (where winds and hence mass loss are weaker) will tend to have higher mass than other black holes. If so, this gives us valuable information about the evolution of these objects. 
Thus, overall, the study of neutron stars and stellar-mass black holes, and especially their masses and spins, connects to many frontier issues of fundamental physics and astrophysics. We now discuss some of the general theory of these objects, from their formation to their later evolution.

\subsection{General theory of neutron stars and black holes}

In the current universe, neutron stars and stellar-mass black holes are most commonly created via core-collapse supernovae. More rarely, black holes could be formed due to the merger of two neutron stars, and it has been suggested that the accretion-induced collapse of a white dwarf could create neutron stars (see [33] and subsequent references), although observational confirmation remains elusive. It has also been proposed that in the early universe primordial black holes might have formed, particularly during periods of phase change such as the quark-hadron transition [34, 35, 36, 37, 38, 39, 40, 41, 42], but there is no evidence for such primordial black holes. We thus focus on the core-collapse formation route after we lay out some basic physical scales.

Basic scales. - The range of known neutron star masses is $1.25 M_{\odot}-$ $2.01 M_{\odot}$ (PSR J0737-3039B [43] and PSR J0348+0432 [44], respectively; here $M_{\odot}=1.989 \times 10^{33} \mathrm{~g}$ is the mass of the Sun). Here, and throughout this review, we use "mass" to refer to the gravitational mass of the object (i.e., the mass that would be inferred using Kepler's laws and observations of the orbit of a distant satellite) instead of the baryonic mass (the mass one would obtain by adding together the separate masses of every constituent particle in the star). The difference between the two is the gravitational binding mass-energy of the star; for a neutron star, the baryonic mass is expected to be 20-30\% larger than the gravitational mass.

Neutron star radii are not well known; estimates from nuclear theory suggest radii in the range of $\sim 10-15 \mathrm{~km}$ [45]. Here we mean the circumferential radius, i.e., the radius obtained by dividing a local measurement of the equatorial circumference by $2 \pi$, rather than other measures of the radius such the proper distance from the center of the star (which can be different by tens of percent). Precise measurements of the radius are coveted by nuclear physicists, because a well-known radius and mass for a neutron star would provide important clues about the nature of the matter in the core. Unfortunately, the systematic errors in current radius estimates are so large that they do not provide useful inputs to nuclear theories ([16]; see [46] for a discussion in the context of quiescent low-mass X-ray binaries). As we will 
discuss more in $\S 3$, the known spin frequencies of neutron stars range from millihertz to hundreds of Hertz, with the current record holder at $716 \mathrm{~Hz}$ (PSR J1748-2446ad, in the globular cluster Terzan 5 [47]). Interestingly, the maximum possible spin frequency of a typical neutron star, beyond which a gravitationally-bound object would fly apart due to centrifugal acceleration, is probably $\sim 1500-2000 \mathrm{~Hz}$ [48]. Thus, as we will explore further in $\S 3$, something limits the spin (probably magnetic torques during accretion).

Black holes, being objects of pure gravity, have no characteristic mass. For example, a sufficiently dedicated and powerful alien species could presumably create mountain-mass black holes, and as we said above such holes might have been produced in the early universe. The masses of known black holes range from a few to tens of solar masses (the stellar-mass black holes that are our focus) to the galactic central black holes that are millions to billions of solar masses. There is an intriguing gap at hundreds to thousands of solar masses that might be filled by intermediate-mass black holes [49], but currently there is no dynamical measurement of such a mass, unlike in the other two mass ranges.

Isolated black holes have only three possible parameters: mass, angular momentum, and electric charge. The net charge is always negligible for astrophysical black holes, as it is for any macroscopic object, because so many free charges exist in astrophysical settings that they would quickly accrete to cancel out the charge. The angular momentum $J$ of a black hole is usually parameterized by the dimensionless combination

$$
\hat{a}=c J / G M^{2}
$$

For a black hole, $0 \leq|\hat{a}| \leq 1$. This is necessary for the hole to have a horizon, but note that it is not an overall limit for all objects: for example $\hat{a}$ for the Earth is $\sim 10^{3}$. The value of $\hat{a}$ for a maximally rotating neutron star is $\sim 0.7$ [48], assuming that the mass is below the stable mass for a nonrotating star. A value of $\hat{a}=0.998$ is sometimes used as an "astrophysical upper limit" for black holes, after work by Kip Thorne [50] showed that matter spiraling into a more rapidly rotating black hole would be spun down by photons emitted retrograde to the spin. This apparently small change, from $\hat{a}_{\max }=1$ to $\hat{a}_{\text {max }}=0.998$, has substantial consequences for the maximum efficiency of energy generation (lowering it to $\sim 32 \%$ from $\sim 40 \%$ ) and other properties. When magnetic fields in accretion disks are taken into account, the maximum spin could be lowered yet further, to perhaps $\hat{a}_{\max } \sim 0.9$ [51, 52]. Any 
spinning object drags reference frames around with it, and near a black hole this can produce pronounced effects.

In standard Boyer-Lindquist coordinates, where the angles $\theta$ and $\phi$ are as usual the colatitude and azimuth, the radius $r$ is the circumferential radius, and intervals in the time $t$ are the intervals between events as measured by a distant observer in flat spacetime, the radius of the event horizon is

$$
r_{H}=\left[1+\left(1-\hat{a}^{2}\right)^{1 / 2}\right] G M / c^{2} .
$$

Another important radius, which features prominently in estimates of the spins of black holes, is the radius of the innermost stable circular orbit (ISCO). In general relativity, unlike in Newtonian gravity, orbits with less than a certain radius around a point mass are not stable. Heuristically, one can think of this as stemming from the greater gravitational accelerations that exist in general relativity; a particle in a circular orbit must go faster (in some sense) closer to the hole than it would in Newtonian gravity. Thus the angular momentum of a circular orbit as a function of radius has a minimum (unlike in Newtonian gravity, where the angular momentum decreases monotonically with decreasing radius). Inside the ISCO, then, small perturbations tend to move fluid elements in a rapidly-opening spiral towards the hole. The circumferential radius of the ISCO is given by [53]

$$
\begin{aligned}
r_{\mathrm{ISCO}} & =G M / c^{2}\left\{3+Z_{2} \mp\left[\left(3-Z_{1}\right)\left(3+Z_{1}+2 Z_{2}\right)\right]^{1 / 2}\right\} \\
\text { where } & \\
Z_{1} & \equiv 1+\left(1-\hat{a}^{2}\right)^{1 / 3}\left[(1+\hat{a})^{1 / 3}+(1-\hat{a})^{1 / 3}\right] \\
Z_{2} & \equiv\left(3 \hat{a}^{2}+Z_{1}^{2}\right)^{1 / 2} .
\end{aligned}
$$

Here the - sign in the expression for $r_{\text {ISCO }}$ is for prograde orbits with respect to the black hole spin, and the + sign is for retrograde orbits. Thus a maximally spinning black hole with $\hat{a}=1$ has $r_{\mathrm{ISCO}}=G M / c^{2}$ for prograde orbits and $r_{\mathrm{ISCO}}=9 G M / c^{2}$ for retrograde orbits, and a nonspinning, or Schwarzschild, black hole has $r_{\mathrm{ISCO}}=6 G M / c^{2}$. The heuristic explanation for the role of spin is that frame-dragging gives prograde orbits a free centrifugal boost, so the angular momentum of a circular orbit at a given radius is less than it would be around a nonspinning black hole. Thus the minimum angular momentum orbit is pushed in for prograde orbits, and is likewise pushed out for retrograde orbits. See Figure 1 for a plot of the horizon radius, ISCO radius, and specific binding energy at the ISCO for the range of possible black hole spin parameters. 


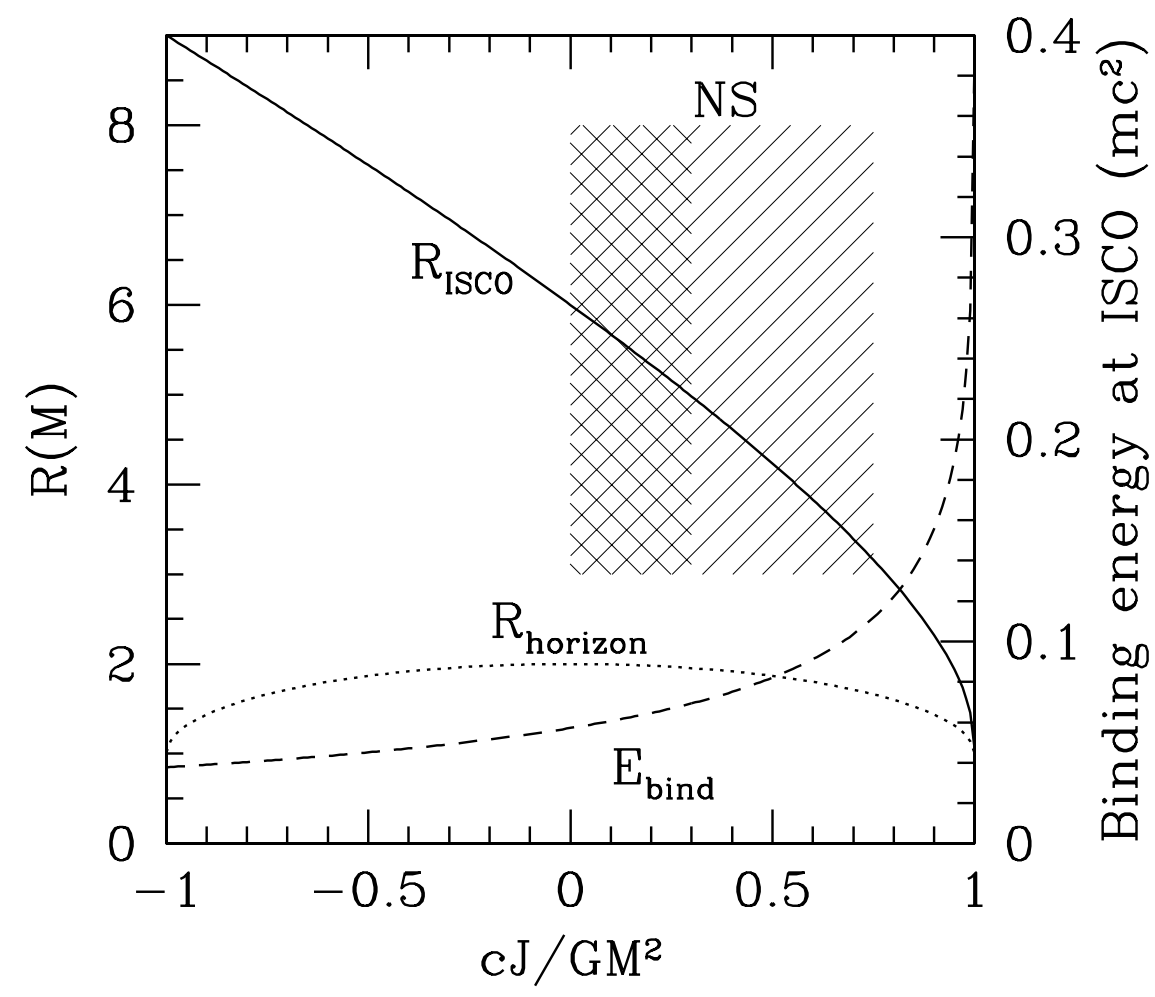

Figure 1: This figure shows the dependence of the radius of the innermost stable circular orbit (ISCO, cast in units of $\left.G M / c^{2}\right)$ on the spin parameter $\left(\hat{a}=c J / G M^{2}\right)$ of a compact object, as well as the radius of the event horizon and the specific binding energy of a test particle in orbit at the ISCO. Typically, the object of concern is a black hole, but as the hatched region shows the size of neutron stars relative to their ISCO is an open and interesting question; here the darker hatching shows a plausible range of radii and spin magnitudes for observed stars, and the lighter hatching extends to the possible range of spin magnitudes. In this plot, positive values of the spin parameter correspond to prograde orbits, whereas negative values correspond to retrograde orbits. 
Core-collapse supernovae.-As roughly five decades of supernova simulations attest, it is difficult to include all the relevant physics in such a simulation. For example, new phenomena emerge in three dimensions compared to two dimensions (e.g., the direction in scale of turbulent cascades reverses; see [54] for a recent discussion of this and other issues in core-collapse theory and simulations). Neutrino transport, rotation, magnetic fields, and accurate nuclear networks all play an important role. One way to understand the delicacy of this seemingly indelicate phenomenon is to note that the photon energy from core-collapse supernovae is $\sim 10^{48}-10^{49} \mathrm{erg}$, and the kinetic energy observed from core-collapse supernovae is commonly $\sim 10^{51} \mathrm{erg}$, whereas the total gravitational binding energy released in the formation of a neutron star is a few times $10^{53} \mathrm{erg}$. Thus only a small fraction of the energy, which is released mainly in neutrinos, couples to the matter. Thus, rather surprisingly for such an energetic event, this is a finely tuned process.

One consequence of the uncertainty in the simulations is that we do not have a firm first-principles grasp of the location of the "mass cut" (the boundary between matter that falls back and matter that escapes in a given collapse/explosion), or the expected rotation rate, or the expected strength of the magnetic field in a neutron star (which affects subsequent spinup and spindown). A common argument still found in textbooks is that the typical birth spin rate and magnetic moment of a neutron star can be obtained by imagining that the Sun collapses and conserves its angular momentum and magnetic flux. Noting that helioseismology suggests a Solar angular momentum of $J_{\odot} \sim 2 \times 10^{48} \mathrm{~g} \mathrm{~cm}^{2} \mathrm{~s}^{-1}$ (e.g., [55]) and that a reasonable guess for the moment of inertia of a neutron star is $I \sim M R^{2} \sim$ few $\times 10^{33} \mathrm{~g}\left(10^{6} \mathrm{~cm}\right)^{2} \sim$ few $\times 10^{45} \mathrm{~g} \mathrm{~cm}^{2}$ gives an angular velocity of $\omega=J / I \sim$ few $\times 10^{3} \mathrm{rad} \mathrm{s}^{-1}$, or a rotation rate of several hundred $\mathrm{Hz}$, consistent with the inferred birth spin rate of, say, the Crab pulsar. Similarly, magnetic flux conservation during the collapse of the Sun to a $10 \mathrm{~km}$ radius object would imply a current surface dipolar field of $B \sim 10^{12} \mathrm{G}$, pleasingly close to the inferred fields of young pulsars.

Unfortunately, this agreement is spurious. The Sun will not collapse to form a neutron star. Instead, it is the core of a massive star that will collapse. This core is approximately only the size of a white dwarf (i.e., at most a few thousand kilometers), which means that even if magnetic flux has been generated it is likely to be insufficient to explain what we see in neutron stars. In addition, the likely coupling of the core to the envelope of the pre-supernova giant [56] suggests that the core might actually rotate quite 
slowly, on $\sim$ year timescales, meaning that when it contracts from $\sim 10^{3} \mathrm{~km}$ to the $\sim 10 \mathrm{~km}$ neutron star the star would be rotating much more slowly than we see in practice. Thus other processes are involved at birth, e.g., off-center kicks [56] for the spin, and probably some dynamo action for the magnetic field [57], although here unfortunately the natural field strength is near equipartition, which would imply $B \sim 10^{17-18} \mathrm{G}$ ! Thus supernova simulators still rely heavily on observational guidance.

Accretion processes.-After the initial core collapse, neutron stars and black holes can acquire additional mass via accretion. Accretion from the interstellar medium will be negligible in almost all circumstances. To see this, note that the standard Bondi-Hoyle rate of accretion from a medium with a density $\rho$, sound speed $c_{s}$, and relative bulk speed $v$ at infinity is

$$
\dot{M}=\lambda \pi \rho\left[\frac{G^{2} M^{2}}{\left(c_{s}^{2}+v^{2}\right)^{3 / 2}}\right]
$$

where $\lambda$ is an eigenvalue of order unity that depends somewhat on the equation of state of the gas. One might think that this rate could be substantial; for example, a $10 M_{\odot}$ black hole in a dense and cold molecular cloud $\left(\rho=10^{-20} \mathrm{~g} \mathrm{~cm}^{-3}\right.$ and $c_{s} \sim v \sim 1 \mathrm{~km} \mathrm{~s}^{-1}$ ) has an accretion rate of $\sim 10^{-6} M_{\odot} \mathrm{yr}^{-1}$, enough to cause e-folding of the mass in $\sim 10^{7} \mathrm{yr}$. However, the accretion will produce a large amount of luminosity that will strongly heat the accreting matter (thus increasing $c_{s}$ ) and pressure balance will then decrease the density (see [58, 59] and many other references).

Significant accretion therefore requires a companion. In some cases the neutron star or black hole might spiral into the companion, producing a Thorne-Żytkow object [60, 61, 62]. Then the accretion rate can be far above the usual Eddington limit

$$
\dot{M}_{\mathrm{Edd}} \equiv L_{\mathrm{Edd}} /\left(\eta c^{2}\right)=\frac{4 \pi G M c}{\kappa} /\left(\eta c^{2}\right) \sim 10^{18} \mathrm{~g} \mathrm{~s}^{-1}\left(M / M_{\odot}\right)(\eta / 0.1)^{-1}
$$

where $\kappa$ is the opacity, $\eta$ is the radiative efficiency, and for our numerical estimate we have assumed fully ionized matter and a Thomson opacity; $\eta \sim$ 0.1 is reasonable for moderately rotating black holes. A rate of accretion much larger than $\dot{M}_{\text {Edd }}$ can lead to radiation that is trapped by the advecting flow [63, 64] in black holes, or can lead to the production of a large number of neutrinos that then escape [65, 66] in neutron stars. A process like this must happen during supernova fallback, and presumably can change the mass or 
the spin of a neutron star or black hole on short timescales. However, this process does not leave the neutron star or black hole with a companion unless the compact object was in a triple system. We will ignore this possibility henceforth.

Mass and spin evolution due to accretion.- Stable mass accretion from a companion to a neutron star or black hole comes in two flavors, depending on the mass of the companion. In a low-mass X-ray binary (LMXB), the companion's mass is typically $M<0.5 M_{\odot}$, and the mass is transferred via Roche lobe accretion. In a high-mass X-ray binary (HMXB), the companion's mass is typically several solar masses, and the mass is transferred when the neutron star or black hole captures part of the wind from the companion.

The distinction is in part because, if all the angular momentum is retained in the system, transfer of mass from the lighter to the heavier member of a binary widens the binary, whereas transfer of mass from the heavier to the lighter tightens the binary. To see this, note that if we have a binary with masses $M_{1}$ and $M_{2}$, which therefore has total mass $M=M_{1}+M_{2}$ and reduced mass $\mu=M_{1} M_{2} / M$, the angular momentum in a circular orbit of radius $r$ is $L=\mu \sqrt{G M r}$. Transfer from the less massive to the more massive object keeps $M$ constant but reduces $\mu$, which thus requires that $r$ increase to compensate. Transfer from the more massive to the less massive object increases $\mu$, so $r$ must decrease. One consequence is that in a binary in which the donor star is more massive than the neutron star or black hole, if the mass donation were through the Roche lobe then the system would shrink, leading to a very large (and thus short lived) mass transfer episode unless the donor shrank rapidly as it lost mass. LMXBs, in contrast, are stable accretors and can last for hundreds of millions of years. Theoretically a compact object could capture mass from the wind of a low-mass companion, but other than during the giant phase these winds are negligible.

HMXB lifetimes are limited to the lifetime of the high wind loss portion of the already short lifetime of a massive star. Thus HMXBs likely live for only tens to hundreds of thousands of years, and transfer $<10^{-2} M_{\odot}$ [67]. In contrast, LMXBs can transfer up to the original mass of the companion, which might reach $\sim 1 M_{\odot}$ in some cases. Another difference is that Roche lobe accretion is accretion with reasonably high angular momentum, so the mass flows in an accretion disk. Winds, in contrast, emerge at roughly the escape speed of the star, because the radiative acceleration that drives them will do so until the wind escapes, and the acceleration is less effective at larger distances so when the speed reaches approximately the escape speed 
the wind is already far from the star. Given that the companion in an HMXB can dominate the total mass of the system, the escape speed from the companion is at least as large as the orbital speed, and it can be much larger if the separation between the companion and the compact object is many times the radius of the companion. Thus the donated matter need not have enough angular momentum to form a disk, and stochastic changes in the wind can alter the direction of the accretion flow [68]. Hence the short lifetimes of HMXBs means they transfer less total mass than LMXBs do, and the mass that is accreted may not be as ordered in its flow.

These considerations have led X-ray astronomers to believe for decades (see [69] for a recent discussion) that the masses and spin magnitudes of stellar-mass black holes are at best moderately affected by accretion from a binary companion. The argument is that even the $\sim 0.5 M_{\odot}$ maximum that could be accreted in an LMXB is a small fraction of the $\sim 10 M_{\odot}$ canonical black hole mass, and integration of the angular momentum equations at the ISCO indicates that the spin magnitude also does not change much (although purely retrograde accretion of $0.5 M_{\odot}$ onto a maximally spinning $\sim 10 M_{\odot}$ black hole could spin it down to $\left.\hat{a} \sim 0.8\right)$. Thus the masses and spin magnitudes of stellar-mass black holes are close to what they were after the supernova.

In contrast, neutron star masses and spin magnitudes could be changed dramatically by accretion. A neutron star of initial mass $M \sim 1.5 M_{\odot}$ that accreted $\sim 0.5-1 M_{\odot}$ might even be pushed over its limit and become a black hole. The spin will change even more substantially. Unlike uncharged black holes, neutron stars have large magnetic fields, so the ionized matter that accretes onto them will couple to the fields. The accretion thus has a large effective lever arm. The coupling radius (sometimes called the Alfvén radius or magnetospheric radius) is

$$
r_{\text {mag }} \approx 4 \times 10^{8} \mathrm{~cm}\left(B / 10^{12} \mathrm{G}\right)^{4 / 7}\left(\dot{M} / 10^{17} \mathrm{~g} \mathrm{~s}^{-1}\right)^{-2 / 7}
$$

assuming a dipolar magnetic field. This is enormously larger than the $\sim$ $10^{6} \mathrm{~cm}$ radius of the star, so accretion can very rapidly spin the star up or down, and what we see now is unrelated to the spin of the neutron star at birth. Isolated neutron stars also spin down, by magnetic braking, which in the pure dipolar case causes the spin period $P$ to evolve as

$$
P^{2}=P_{0}^{2}+2 P \dot{P} T
$$


Here $T$ is the age of the pulsar, $P_{0}$ is its initial period, and $P \dot{P} \propto B^{2}$ in the magnetic dipole model. Thus $P \dot{P}$ is constant if the field strength and geometry do not change. When $2 P \dot{P} T \gg P_{0}^{2}$, the characteristic age is thus $\tau=P /(2 \dot{P})$. Thus the current spin frequency of an isolated neutron star is only a lower limit on its birth frequency. As we discuss further in $\S 3$, estimates have been made of the birth frequencies of some neutron stars based on the observation that the spindown energy would have to go into the surrounding nebula, so initial frequencies that are too high would lead to nebulae that are too bright.

The direction of the spin of an accreting neutron star is determined by the accretion, for the reasons indicated above. The case is not as clear for accreting black holes. Another consequence of the frame dragging around spinning black holes is that matter orbiting in a different plane than the black hole spin plane will have its orbital axis precess around the black hole spin axis. When there is dissipation, this will lead to a gradual settling of the matter's motion into the spin plane of the hole (the orbits could be prograde or retrograde, depending on the conditions; see [70]), a process called the Bardeen-Petterson effect [71]. The black hole spin direction is thus also changed in response. The relatively small size of the black hole means that its specific angular momentum is small compared to the specific angular momentum of the disk; a rough ratio is $\ell_{\mathrm{BH}} / \ell_{\text {disk }} \approx \hat{a}\left(r_{\mathrm{BH}} / r_{\text {disk }}\right)^{1 / 2}$. Thus for a few $\times 10^{10} \mathrm{~cm}$ disk around a $10^{6} \mathrm{~cm}$ black hole, the integrated angular momentum of the matter that flows through the disk will be greater than in the black hole even if total accreted mass is only $\sim 1 \%$ of the black hole mass (i.e., about $0.1 M_{\odot}$ ).

The reason that this does not guarantee alignment is that much of the disk angular momentum actually flows outwards, where it is ultimately transferred to the orbital angular momentum of the companion. The relevant radius for black hole alignment is where the disk inclination bends towards the black hole spin plane. There is considerable discussion of where this is [72, 73, 74, 75, 76, 77, 78, 79, 80, 81, 82], with typical estimates being hundreds to thousands of times the gravitational radius $r_{g} \equiv G M / c^{2}$, but it may also be that alignment is less effective when the accretion rate approaches Eddington [83]. Thus the current orientation of the black hole spin might retain some memory of its birth orientation rather than being fully aligned with the orbit. As we will discuss in $\S 3$, this consideration, and the disputed evidence that misalignment is seen in some systems, has significant implications for spin estimates using X-ray continua. 
We now discuss the methods of estimating the masses of neutron stars and black holes, and the implications of the results.

\section{Masses}

Ever since the 1767 suggestion by John Michell, the intellectual grandfather of black holes, that some visually close stars are actually physically associated with each other [84], binaries have been prime sources for the determination of the masses of their components. This is because Kepler's laws can be used directly for such systems. Other techniques are sometimes applied; for example, asteroseismology has been used to find the masses of some white dwarfs, main sequence stars, and giants [85, 86, 87, 88]. But neutron stars and black holes have neither observed oscillations nor confirmed surface atomic lines (which provide critical information for ordinary stars). Future mass measurements using microlensing are promising (see [89] for a recent review, including the discussion of several black hole candidates), particularly if precise astrometric measurements can break degeneracies between the mass, proper motion, and distance to the lens. However, the current sample of measured masses is dominated by compact objects in binaries.

In this section we begin with a theoretical discussion of the maximum mass of a neutron star. We then describe the mass function, which places a lower limit on the mass of the companion to an observed object in a binary, then discuss some of the post-Keplerian observables that can be seen in binary pulsar systems. We finish by giving references for the masses and estimated uncertainties for a number of neutron stars and black holes.

\subsection{The maximum mass of a neutron star}

The mass boundary between neutron stars and black holes is important for two reasons. First, it is sensitive to the state of cold matter beyond nuclear density; laboratory experiments cannot access this realm, and nuclear models disagree strongly about the likely properties of such matter. Second, as we discuss at the end of this section, a mass measurement above the neutron star maximum is the best way to establish that a compact object is a black hole, because other proposed discriminants are unreliable. Here we focus for simplicity on nonrotating neutron stars, with some comments at the end about the role of uniform and differential rotation.

As our first guess about the maximum mass of a neutron star we can use the elementary Landau [90] derivation of Chandrasekhar's [91] maximum 
mass for white dwarfs. Suppose that we have a fermion (an electron for white dwarfs, a neutron for neutron stars) whose degeneracy pressure holds up a cold, dense, spherical object. If the number density of this fermion is $n$ then a given fermion is localized within a region of size $\Delta x \sim n^{-1 / 3}$ and its Fermi momentum is

$$
p_{F} \sim \hbar / \Delta x \sim \hbar n^{1 / 3} .
$$

The Fermi energy per fermion is $E_{F} \approx p_{F}^{2} / 2 m_{F}$ in the nonrelativistic case (i.e., when $p_{F} \ll m_{F} c$ ), where $m_{F}$ is the mass of the fermion, and $E_{F} \approx p_{F} c$ in the relativistic case. Now suppose that there are $\mu_{F}$ massive particles (protons and/or neutrons) per fermion; thus $\mu_{F} \sim 2$ for white dwarfs and $\mu_{F} \sim 1$ for neutron stars. In a star of total mass $M$ and radius $R$, this means that the average number density is $n \sim M /\left(\mu_{F} m_{n} R^{3}\right)$, where $m_{n}$ is the mass of a neutron. Thus $E_{F} \sim\left(\hbar^{2} / 2 m_{F}\right)\left[M /\left(\mu_{F} m_{n} R^{3}\right)\right]^{2 / 3} \sim 1 / R^{2}$ for nonrelativistic degeneracy, and $E_{F} \sim \hbar c\left[M /\left(\mu_{F} m_{n} R^{3}\right)\right]^{1 / 3} \sim 1 / R$ for relativistic degeneracy.

Ignoring general relativistic corrections and structural details of the star, the gravitational potential energy per degenerate fermion is

$$
E_{\text {grav }} \sim-\frac{G M m_{n} \mu_{F}}{R} .
$$

Thus when we highlight the dependences on the mass, radius, and $\mu_{F}$, the total energy per degenerate fermion in the nonrelativistically degenerate case is

$$
E_{\mathrm{tot}}=E_{F}+E_{\mathrm{grav}}=\frac{C_{1} M^{2 / 3}}{\mu_{F}^{2 / 3} R^{2}}-\frac{C_{2} M \mu_{F}}{R}
$$

where $C_{1}$ and $C_{2}$ are dimensional constants. From this expression we see that minimization of the energy leads to a stable configuration; too large a radius asymptotes the energy to zero (contrasted to the minimum energy, which is negative because the star is bound), and too small a radius yields a positive energy. We also see from energy minimization that at equilibrium, $R \propto M^{-1 / 3}$, so we recover the result that degenerate objects are smaller when they are more massive.

In the relativistically degenerate case,

$$
E_{\mathrm{tot}}=\frac{C_{3} M^{1 / 3}}{\mu_{F}^{1 / 3} R}-\frac{C_{2} M \mu_{F}}{R} .
$$


Both terms have the same $R$ dependence, so if the expression is positive at any $R$ the star will expand to lower its energy until it becomes nonrelativistic, at which point it can settle into a stable equilibrium. In contrast, if the energy is negative, it can contract indefinitely to reach lower and lower energies, so the star is not stable. We see that higher masses lead to lower energies at fixed $R$, so the limiting mass is the one that yields $E_{\text {tot }}=0$. This equation shows that $M_{\max } \propto \mu_{F}^{-2}$. The maximum mass for a white dwarf $\left(\mu_{F} \approx 2\right)$ is $M_{\mathrm{WD}} \approx 1.4 M_{\odot}$, so we would expect that the maximum mass for a neutron star $\left(\mu_{F} \approx 1\right)$ would be $\sim 4$ times higher, or $\sim 5.6 M_{\odot}$.

In reality the maximum mass of a neutron star is much smaller than this value. The main reason is that, unlike white dwarfs, neutron stars are compact enough that mass-energy contributions other than the rest mass play an important role. Thus the increased pressure that comes with higher density will itself gravitate, so systems of a given mass are more susceptible to collapse then they would be in Newtonian gravity. A more rigorous approach to the determination of the maximum mass uses the Tolman-OppenheimerVolkoff equation [92]

$$
\frac{d P(r)}{d r}=-\frac{G}{r^{2}}\left[\rho(r)+\frac{P(r)}{c^{2}}\right]\left[M(r)+4 \pi r^{3} \frac{P(r)}{c^{2}}\right]\left[1-\frac{2 G M(r)}{c^{2} r}\right]^{-1} .
$$

This is effectively the equation of hydrostatic equilibrium for a spherically symmetric, nonrotating star in general relativity. Here $M(r)$ is the gravitational mass contained inside a circumferential radius $r$, and $P(r)$ and $\rho(r)$ are respectively the pressure and density at $r$. Given a central density and an equation of state that relates $P$ to $\rho$, one can compute the mass and radius of the star. Note that because the temperature $T<10^{9-10} \mathrm{~K}$ inside a neutron star with an age of at least hundreds of years is much less than the Fermi temperature $T_{F}=E_{F} / k \sim 10^{12} \mathrm{~K}$, temperature can be neglected in the equation of state. Similarly, because the Fermi energy of hundreds of $\mathrm{MeV}$ in the core is much greater than the energy differences per particle ( few $\mathrm{MeV}$ ) between different compositions, it is usually assumed that the core matter is in its (unknown) equilibrium composition.

Thus given $P(\rho)$, one can calculate the maximum mass of a neutron star. However, because the central densities are at several times nuclear saturation density (i.e., out of reach of laboratory experiments) and the matter is strongly skewed towards neutrons rather than protons (in contrast to the nearly-symmetric nuclear matter in normal nuclei), $P(\rho)$ is not known from 
first principles. One might imagine that the equation of state could be calculated from quantum chromodynamics. However, because fermion wavefunctions switch sign when particles are swapped, estimates of the total energy of a collection of fermions require evaluation of a sum of nearly cancelling terms (this is known as the fermion sign problem, e.g., [93, 94, 95, 96]). Thus the ground state of nuclear matter, especially under significant pressure, is exponentially difficult to compute from first principles. Thus nuclear physicists rely on astronomers to determine observationally the masses and, ideally, the radii of neutron stars.

The maximum mass of neutron stars can be constrained theoretically using an approach pioneered by Rhoades and Ruffini ([97]; see also [98]). They noted that we do have a good understanding of the state of matter from zero density up to nuclear saturation density $\left(\rho_{s} \approx 2.6 \times 10^{14} \mathrm{~g} \mathrm{~cm}^{-3}\right)$. They showed that at higher densities the equation of state that gives the largest possible mass is one that is at maximum stiffness, i.e., one for which the speed of sound $c_{s}=(d P / d \rho)^{1 / 2}$ is the speed of light. Applying that assumption to all densities above $\rho_{s}$ gives a maximum mass of $M_{\max } \approx 3.2 M_{\odot}$. One can extend this to ask: if we know the equation of state up to some density and then use a $c_{s}=c$ equation of state, what is the maximum mass? Kalogera and Baym [98] showed that if we trust the (largely converged and calibrated) equation of state up to $2 \rho_{s}$, the maximum mass drops to roughly $M_{\max }=$ $2.9 M_{\odot}$. Astronomers thus use the rough criterion that if an X-ray emitting object in a binary exceeds $3 M_{\odot}$, it is most likely a black hole rather than a neutron star. As a note of caution, even at $\rho_{s}$ neutron star matter has far more neutrons than protons, so what we can measure in laboratories (see [99] for a recent review) does not provide absolutely definitive constraints on the relevant highly asymmetric matter.

Rotation adds centrifugal support, so rotation can increase the maximum mass of a neutron star, by as much as $\sim 25 \%$ [100]. Indeed, there are studies of both supermassive neutron stars (uniformly rotating stars that would be unstable if they stopped rotating) and the yet more massive hypermassive neutron stars (differentially rotating stars that would be unstable if their angular momentum were redistributed to yield uniform rotation). Such stars could have masses a few tenths of a solar mass in excess of the nonrotating maximum. The hypermassive case is not relevant for the long-lived neutron stars we see, because differential rotation is damped quickly [101]. Supermassive neutron stars are potentially relevant, but in practice the highest rotation rate ever seen from a pulsar $(716 \mathrm{~Hz}$; see [47]) is probably a factor 
of $\sim$ two less than the maximum rotation rate [48]. Centrifugal support is a quadratic effect, so rotation is at best a minor contributor to the maximum mass of observed neutron stars.

\subsection{The binary mass function}

Suppose that one observes a star in a binary, which we will call star 1, over at least one full period. Monitoring of spectral lines (from ordinary stars) or pulses (from a pulsar) can yield both the period $P$ and the maximum line of sight speed $K_{1}$ of star 1 from our perspective. It is straightforward to show using Kepler's third law that for an orbit of eccentricity $e$

$$
f\left(M_{1}, M_{2}, i\right) \equiv \frac{K_{1}^{3} P\left(1-e^{2}\right)^{3 / 2}}{2 \pi G}=\frac{\left(M_{2} \sin i\right)^{3}}{\left(M_{1}+M_{2}\right)^{2}}
$$

(e.g., [102]). Here $M_{1}$ is the mass of star $1, M_{2}$ is the mass of its companion, star 2 , and $i$ is the inclination of the orbit to our line of sight, defined so that $i=0$ for a face-on orbit and $i=90^{\circ}$ for an edge-on orbit.

Examination of this equation shows that the mass function $f$ is a lower limit to $M_{2}$, the mass of the unobserved star. Indeed, $f$ is the actual mass of star 2 only if the system is edge-on and $M_{1} \rightarrow 0$. If the motion of star 2 can also be measured, e.g., in a double-line spectroscopic binary or a double pulsar, then the mass ratio is also known but the inclination remains a source of uncertainty. Even if the second star cannot be observed in this way, if $M_{1} \ll M_{2}$ then the uncertainty its unknown mass introduces is small. This is why high-mass X-ray binaries provide more ambiguous evidence for stellarmass black holes than do low-mass X-ray binaries. For example, the mass function of $\mathrm{Cyg}-\mathrm{X}-1$ is only $\approx 0.25 M_{\odot}$ ([103] and subsequent references), so assumptions must be made about the mass of the companion to make a case that the unseen object exceeds the maximum mass of a neutron star and is thus likely to be a black hole.

The orbital inclination is often difficult to determine for non-eclipsing systems. A method that has found success for Roche lobe filling binaries is the use of ellipsoidal light variations based on the tidal distortion of the donor [104, 105, 106]. The key idea is that a tidally distorted star has a larger cross section as seen from the side than as seen along the line to the compact object, and that the light from when the donor is behind the compact object looks different from when it is in front of the compact object because of edgedarkening effects near the L1 point. A face-on system will obviously show 
no variation in the companion's light with orbital phase, whereas an edge-on system will show maximum variation. Thus the inclination can be estimated even for non-eclipsing systems. The low luminosity of the companion in a lowmass X-ray binary means that this method is best used for transient systems, where the accretion disk essentially goes away completely and hence does not contaminate the light from the companion (e.g., [106]). Perhaps due to their higher mass ratio, black hole LMXBs are more likely to be transient systems than neutron star LMXBs. Thus this method has been used successfully for several black hole systems, but few neutron star systems.

\subsection{Binary pulsars and post-Keplerian parameters}

A method unique to neutron stars that can yield high-precision masses is the careful timing of signals from pulsars. The pulses from pulsars act as superior clocks, with characteristic spindown timescales $P / 2 \dot{P}$ that can be several billion years for millisecond pulsars, the most stable rotators. Thus even minute deviations from slow, steady spindown are highly significant and can be used to infer subtle relativistic effects in the binary system. These considerations led Joseph Taylor and Russell Hulse to search for and discover the first binary neutron star system in 1974 [107]. As we will discuss, the timing of this and similar double neutron star systems has led to precise confirmation of general relativity in weak gravity.

The extra effects that can currently be measured are:

- Precession of the pericenter. To lowest order the frequency of precession of an orbit is

$$
\dot{\omega}=3\left(\frac{P_{b}}{2 \pi}\right)^{-5 / 3}\left(T_{\odot} M\right)^{2 / 3}\left(1-e^{2}\right)^{-1} .
$$

- The Einstein delay. At pericenter, the pulsar is deepest in the gravitational well of the companion, which slows down the received pulse rate. The magnitude of the effect is

$$
\gamma=e\left(\frac{P_{b}}{2 \pi}\right)^{1 / 3} T_{\odot}^{2 / 3} M^{-4 / 3} m_{c}\left(m_{p}+2 m_{c}\right) .
$$

- Binary orbital decay due to gravitational radiation. The rate of decay of the orbital period is

$$
\dot{P}_{b}=-\frac{192 \pi}{5}\left(\frac{P_{b}}{2 \pi}\right)^{-5 / 3} f(e) T_{\odot}^{5 / 3} m_{p} m_{c} M^{-1 / 3}
$$


- The Shapiro delay. If the pulses pass close to the companion, the clock of the pulses experiences time dilation. A face-on orbit will have no dependence of the Shapiro delay on orbital phase, whereas a nearly edge-on orbit will have a sharp increase in the delay at conjunction. Thus in addition to the magnitude of the effect (the range, or $r$, parameter), there is an effect (the shape, or $s$, parameter) that depends on the binary orientation:

$$
\begin{aligned}
r & =T_{\odot} m_{c} \\
s & =\sin i .
\end{aligned}
$$

Here we use the notation of [108]. In this list, $m_{p}$ is the pulsar mass, $m_{c}$ is the companion mass, $M=m_{p}+m_{c}$ is the total mass (all masses are in solar units), $T_{\odot} \equiv G M_{\odot} / c^{3}=4.925590947 \mu \mathrm{s}, P_{b}$ is the binary orbital period, and $f(e)=\left(1+73 e^{2} / 24+37 e^{4} / 96\right)\left(1-e^{2}\right)^{-7 / 2}$. Neutron stars are so small compared to their separations in these systems $\left(\sim 10^{6} \mathrm{~cm}\right.$ versus $\sim 10^{11} \mathrm{~cm}$ ) that they are essentially point sources and there are thus no tidal effects etc. to confuse the analysis of these binaries. A given system has five associated parameters (two masses and the inclination, semimajor axis, and eccentricity), so when there are more than five independent observables the system is overdetermined and the underlying theory can be tested.

The neutron stars in double neutron star systems (when at least one appears to us as a pulsar) have masses that can be measured to $\sim 10^{-3}$ relative precision. The pulsars in these systems rotate with periods of tens of milliseconds, and are not as stable as the true millisecond pulsars (some of which have timing stability one or two orders of magnitude greater; see [109]). If a millisecond pulsar were to be found in a close binary with another neutron star, the gain in timing precision would eventually allow the measurement of higher-order effects (see [110] for a discussion in the context of the double pulsar PSR J0737-3039).

However, because millisecond pulsars are spun up by accretion of at least several hundredths of a solar mass from a companion, and the high-mass stars that are the progenitors of neutron stars do not last long enough to donate such mass [67], millisecond pulsars are instead found in binaries with white dwarfs or main sequence stars. These objects are large enough that tidal effects can be significant. In addition, the accretion process circularizes the orbit (but not completely; see [111] for a remarkable demonstration using the fluctuation-dissipation theorem that residual eccentricities of a few parts 
per million can be produced, depending on the binary orbital period), so it can be difficult to measure pericenter precession or the Einstein delay, both of which require nonzero eccentricity. Nonetheless, recently two neutron stars in such systems have been found to have masses $M \approx 2 M_{\odot}$, which as we will discuss in the next section has important implications for dense matter.

The first of these, PSR J1614-2230 [112], is a pulsar-white dwarf system that had its Shapiro delay measured due to its fortunate nearly edge-on orientation. For a circular orbit, the two parameters of the Shapiro delay plus the two Keplerian parameters (orbital period and line of sight speed) suffice to determine both masses. The orientation is also important because for edge-on inclinations the Shapiro delay as a function of orbital phase acquires a cusp near conjunction; more moderate inclinations produce a phase dependence that is partially degenerate with what one would find in an orbit with moderate eccentricity. The mass inferred for PSR J1614-2230 was $M=1.97 \pm 0.04 M_{\odot}[112]$.

More recently, Antoniadis et al. [44] have found a mass of $M=2.01 \pm$ $0.04 M_{\odot}$ for PSR J0348+0432. This is another pulsar-white dwarf system, but the method of mass estimation was different. In addition to the pulsar frequency modulation, the authors were able to measure orbital line energy variations from the white dwarf. This immediately gives a mass ratio between the neutron star and the white dwarf. They estimated the mass of the white dwarf based on white dwarf models and the measured gravitational redshift of the lines, leading to the final mass estimate for the neutron star.

A convenient updated list of neutron star masses and their uncertainties is maintained at http://www.stellarcollapse.org/nsmasses by Jim Lattimer and Andrew Steiner. From this it is apparent, and it was noted early, that the masses of neutron stars in double neutron star systems are very tightly clustered, around $1.35 \pm 0.05 M_{\odot}$. Indeed, prior to the discovery of more massive neutron stars in binaries with white dwarfs, this clustering was sometimes used to argue that the maximum mass of neutron stars is low, around $1.5 M_{\odot}$ [113]. It now seems clear that if this clustering is significant it stems from a narrowness of evolutionary channels rather than from the fundamental physics of neutron stars. We also note that millisecond pulsars seem to have systematically larger masses than neutron stars that are not likely to have accreted much [114]. This accords with our expectation that millisecond pulsars could accrete up to several tenths of a solar mass in their lifetimes.

For an early list of estimated black hole mass functions, see [115]; for 
more recent updates, see [116, 117], and for an object-by-object discussion see Section 5 of [9]. At face value it appears that there is a gap between the masses of neutron stars and black holes [116, 117], although there have been recent concerns raised about the role of selection effects [9]. If there is indeed a gap, one possible explanation is that black holes form from failed supernovae and thus have much greater fallback than neutron stars [118]. More observations are necessary. In particular, if there is a large gap in the chirp mass distribution inferred from the gravitational waves emitted in compact binary coalescence (see Section 4) this will confirm the mass gap between black holes and neutron stars as well as making it easier to identify which sources contain neutron stars and which contain black holes.

We now turn our attention to the inferred spins of neutron stars and black holes.

\section{Spins}

As we said in Section 1, stellar-mass black holes have spin magnitudes close to what they were at birth. In contrast, we will show that the spin periods of neutron stars typically bear little relation to their periods at birth. In addition, even the fastest-spinning neutron stars have far less angular momentum than stellar-mass black holes. In this section we provide context for these statements by discussing what we know about the spins of neutron stars and black holes. In Section 5 we will return to the question of spins by discussing the ongoing debate about the degree to which black hole spins affect the powers of their jets.

\subsection{Neutron Star Spins}

The spin periods of more than 2500 neutron stars are known from the periodic modulation of their intensities. Of these the vast majority - more than 2300 - are seen in radio bands, and have periods ranging from $1.4 \mathrm{~ms}$ to 12 s (see, e.g., the Australia Telescope National Facility Pulsar Catalog http://www.atnf.csiro.au/research/pulsar/psrcat/ [119] and the cata$\log$ http://astro.phys.wvu.edu/GalacticMSPs/GalacticMSPs.txt of Duncan Lorimer; note that Fermi detections of gamma rays have made a significant contribution to our catalogs of pulsars out of the Galactic plane [120]). These pulsars are thought to be rotation-powered, meaning that the spindown energy of the pulsar is the ultimate power source of the radio emission (even though the radio luminosity is a tiny fraction of the spindown 
power). There are another $\sim 200$ pulsars that are detected from their bright X-ray emission. These pulsars are accretion-powered: the source of energy is the gravitational energy released when matter taken from a binary companion is funneled by the neutron star's magnetic field onto a portion of the neutron star's surface. The spin periods of these pulsars range from $1.7 \mathrm{~ms}$ to at least three hours (see [121]). There are also a handful of other neutron stars that have known periods based on phenomena such as thermonuclear burst oscillations [122] or that are powered by strong magnetic fields rather than rotation (the magnetars; see [123] and http://www.physics.mcgill.ca/ pulsar/magnetar/main.html for a catalog).

For our purposes, the most important question related to neutron star spin is whether a given star is accreting actively. We now discuss the properties of non-accreting and accreting neutron stars and the spin periods and evolution that have been inferred.

\subsubsection{Non-accreting pulsars}

As we mentioned earlier, the discovery of periodic radio sources in 1967 [1] ushered in the era of neutron star observations. Even before the discovery of pulsars, Pacini [125] proposed that magnetic dipole radiation from a spinning magnetic neutron star could power supernova remnants. It was, however, the elegant work of Gold [126] that convinced the community that the periodic pulsations observed by Jocelyn Bell and colleagues were due to rotating neutron stars. At its core, Gold's argument is that (1) the shortest periods known in 1968 were tens of milliseconds, which is only accessible by neutron stars or black holes (even white dwarfs are not compact enough for such periods), (2) black holes have no stable structures that could emit regular beacons, so the objects must be neutron stars, (3) periodic signals can be produced by rotation, pulsation, or orbits, but (4) neutron star pulsations have typical periods of at most a few milliseconds, which could not explain the few-second periods that were then known, and (5) orbital periods would decrease in time, rapidly, due to emission of gravitational radiation, in contrast to the very nearly stable but increasing periods of known pulsars. Thus rotating neutron stars are the only possible objects that could produce such short periods that are also nearly stable but increase with time.

The power source of most of these pulsars is likely to be rotation. As Gold stated:

There are as yet not really enough clues to identify the mechanism of radio emission. It could be a process deriving its energy from some source 


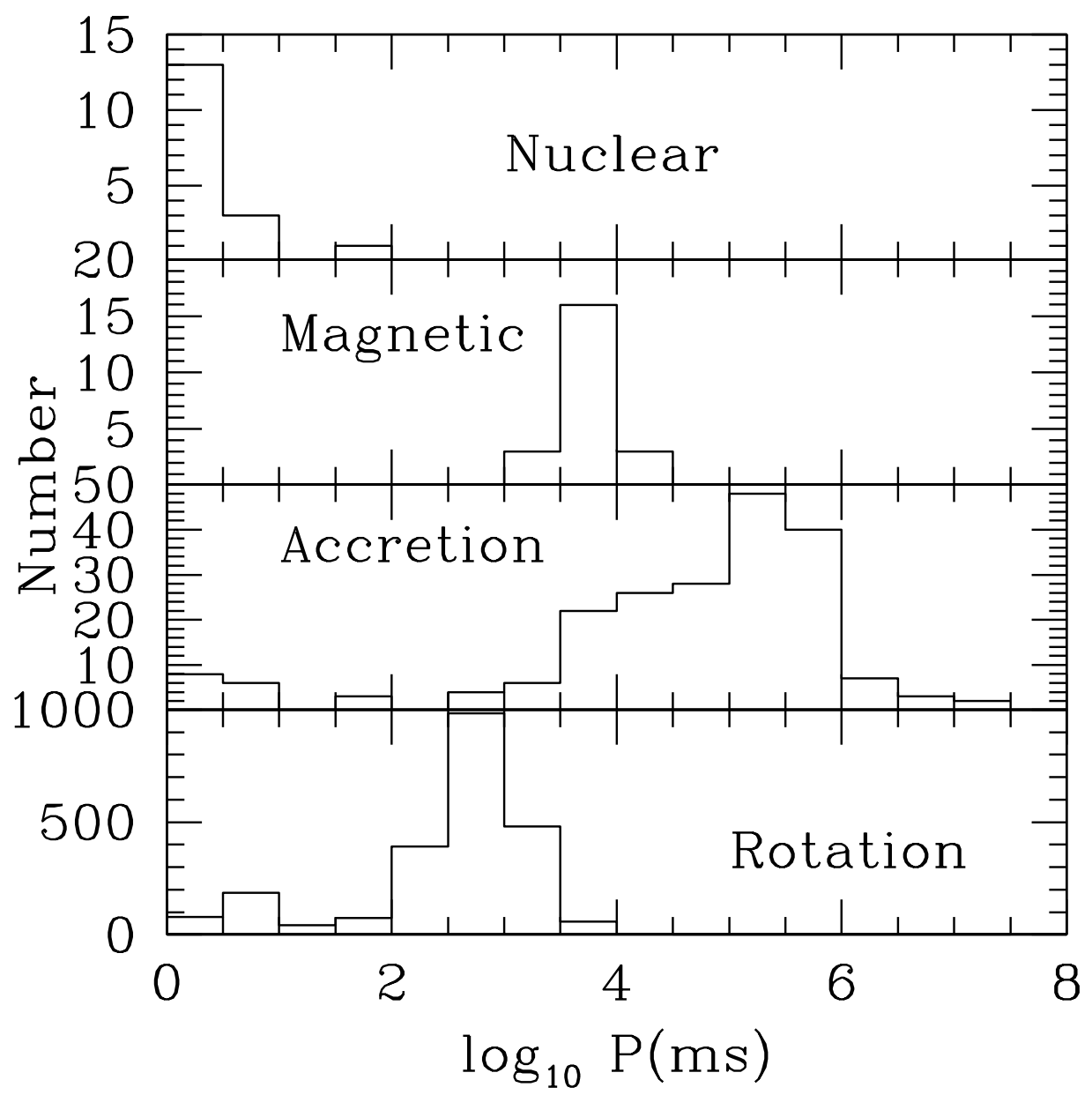

Figure 2: Histograms of neutron star spins, categorized by the primary power source. Some of the nuclear-powered pulsars are also accretion-powered pulsars. There are numerous selection effects that influence the observed distributions, but the general patterns may be representative of the actual sample. Data for the rotation-powered pulsars are from http://www.atnf.csiro.au/research/pulsar/psrcat/ [119], data for accretion-powered pulsars are from http://www.iasfbo.inaf.it/ mauro/pulsar_list.html, data for magnetars are from http://www.physics.mcgill.ca/ pulsar/magnetar/main.html 123], and data for nuclear-powered pulsars are from [124]. 
of internal energy of the star, and thus as difficult to analyse as solar activity. But there is another possibility, namely, that the emission derives its energy from the rotational energy of the star (very likely the principal remaining energy source), and is a result of relativistic effects in a co-rotating magnetosphere.[126]

Note that radio emission carries only a tiny fraction of the spindown power, but higher-energy emission such as gamma rays can sometimes have tens of percent of the spindown luminosity [127]. Indeed, there is a growing category of sources whose integrated X-ray luminosity is considerably greater than the spindown luminosity. These are known as "magnetars", and are thought to be powered by the decay and reconfiguration of internal magnetic fields [128, 129].

Regardless of what powers the observed emission, non-accreting neutron stars are thought to spin down essentially by magnetic dipole radiation (see Section (1). Putting units to equation (7), the period evolves as $P^{2}=P_{0}^{2}+$ $2 B_{12}^{2}\left(T \cdot 10^{-15} \mathrm{~s}\right)$ for an average surface dipolar field of $10^{12} B_{12} \mathrm{G}$. Here $T$ is the current age of the pulsar. This means that memory of the initial spin is lost rapidly if the birth period was much less than a second and the field strengths are in the $\sim 10^{12} \mathrm{G}$ range usually considered for young neutron stars. For example, the Crab pulsar is $\sim 1000$ yr old (the supernova was first seen on 4 July 1054). Its surface field is inferred to be $B_{12} \approx 5$. Thus if the initial period was much shorter than the current period of $33 \mathrm{~ms}$ then the inferred age of $T \approx 700 \mathrm{yr}$ is roughly consistent with the known age. Initial periods anywhere from $\sim 1 \mathrm{~ms}$ to $\sim 10 \mathrm{~ms}$ can be accommodated easily. For older pulsars an even greater range of initial periods are consistent with the data. Thus pulsars older than a few thousand years may well have completely lost memory of their initial period. Indeed, it is evident from Table 1 of [130] that most pulsars in supernova remnants have characteristic ages $T_{c}=P / 2 \dot{P}$ that are consistent within the uncertainties with the age of the remnant. Thus for those pulsars the initial period could have been anything much less than the current period.

We note, incidentally, that the dipole spindown model in its simplest form fails a self-consistency test. For pure magnetic dipole spindown, the "braking index" $n_{b} \equiv \nu \ddot{\nu} / \dot{\nu}^{2}$, where $\nu$ is the spin frequency, is equal to 3. Measured values, however, are always less than 3, sometimes by very significant amounts 131]. This suggests that other processes are at work; see [132, 133] for some recent ideas.

To close this section we mention for completeness that if there is sufficient 
nonaxisymmetry in a rapidly-rotating neutron star, it could be slowed down by emission of gravitational waves (e.g., via R-mode instabilities on the surface of a very young, hot neutron star [134], [135]). However, the efficiency of this mechanism is expected to have a very steep dependence on the initial spin period, and is also expected to have a very low saturation amplitude, so it is not clear if this mechanism will be important. Stars with more modest initial spin periods may take thousands of years to spin down substantially ([136], [137]).

\subsubsection{Accreting pulsars}

A few years after the discovery of rotation-powered pulsars, pulsating Xray sources were discovered in several binaries (e.g., Her X-1; [138]). Initial modeling work did not consider neutron stars because of the assumption that the required supernova would disrupt any binary, but soon several groups proposed that accretion from a companion, guided by the magnetic field of the neutron star, would produce an X-ray emitting hot spot on the surface [139, 140, 141]. Later work established quantitative relations between the spin frequency of an accreting neutron star and its average accretion rate and dipole magnetic moment, based on the idea that a magnetic spin equilibrium could be established on a timescale much shorter than the X-ray active timescale of the source (e.g., [142, 143, 144, 145, 146, 147, 148]). In spin equilibrium, the rotation frequency of an accreting magnetic neutron star is

$$
\nu=0.3 \mathrm{~Hz} \xi \dot{M}_{17}^{3 / 7} \mu_{30}^{-6 / 7}\left(M / M_{\odot}\right)^{5 / 7}
$$

for a star with a magnetic moment $10^{30} \mu_{30} \mathrm{G} \mathrm{cm}^{3}$ and an average accretion rate of $10^{17} \dot{M}_{17} \mathrm{~g} \mathrm{~s}^{-1}$. Here $\xi$ is a factor of order unity that depends on the detailed structure of the flow. Thus for the neutron stars in high-mass Xray binaries, which typically have $\mu_{30} \sim$ few the equilibrium period is often seconds or longer, but for the neutron stars in low-mass X-ray binaries, which usually have $\mu_{30} \sim 10^{-4}-10^{-3}$, periods of milliseconds can be attained via spinup.

Indeed, accretion-induced spinup was quickly suggested to explain the first millisecond pulsar detected [149, 150, 151]. This explanation has been strengthened by the evidence that millisecond pulsars are usually in binaries ( $\sim 70-80 \%$ versus the $\sim 1 \%$ of slower pulsars). The observation of nearly coherent millisecond oscillations in thermonuclear X-ray bursts [122] was taken by most researchers to show that the stars hosting those bursts, which 
are in LMXBs, are the progenitors of the non-accreting millisecond pulsars. However, the frequencies of the burst oscillations drift slightly and thus some doubt remained until the discovery of $401 \mathrm{~Hz}$ accretion-powered oscillations from SAX J1808-3658 [152, 153], which were later tied to the frequency and phase of burst oscillations from this source [154]. Multiple sources now have both accretion-powered and nuclear-powered oscillations at the same frequency. One source has even been found to transition between accretionpowered pulsations and rotation-powered pulsations when the accretion dies off sufficiently [155], and there are other sources for which somewhat more indirect evidence of this phenomenon has been suggested [156, 157].

Currently, there are 15 known accretion-powered millisecond X-ray pulsars (see [121] for a review). This includes the X-ray binary Aql X-1, in which $\sim 150$ seconds of coherent oscillations were discovered among many megaseconds of data gathered with the Rossi X-ray timing explorer (RXTE) ([158]). The observed range of spin frequencies is $\nu=182-599 \mathrm{~Hz}$; seven have binary orbital periods of two hours or shorter.

Given that the number of accreting millisecond X-ray pulsars is growing, and especially given that a non-pulsing X-ray binary was found to have momentary coherent pulsations, the question arises: why are coherent pulsations not found in all neutron star X-ray binaries? The reasons may be complex, multiple mechanisms may work jointly to hide pulsations, and observations have not yet revealed the answer. Alignment of the rotation and magnetic axes could serve to hide pulsations (e.g. [159], [160]). It has also been suggested that the magnetic field is buried by accretion (e.g., [161]), only to re-emerge when the accretion rate drops. This agrees with the observation that accretion-powered millisecond X-ray pulsars are typically underluminous compared to more standard neutron star X-ray binaries. However, it is not clear how to reconcile this explanation with the observation that most non-accreting millisecond pulsars obey the spin-up line [162], which is the line on a $P-\dot{P}$ diagram that would be obeyed for a source accreting at the Eddington rate or below (for a discussion of some of the complexities involved in defining this line, see Section 4.3 of [163]). This suggests that most of the accretion occurred at nearly the current magnetic moment, rather than one that was much lower.

Accretion could also slow down the rate of rotation of a neutron star. As can be seen from Equation (18), if the accretion rate is small enough or the magnetic dipole moment is large enough, the equilibrium period can be hundreds to thousands of seconds. Periods of this size are indeed seen in some 
HMXBs (see http://www.iasfbo.inaf.it/ mauro/pulsar_list.html) and also in a few LMXBs such as symbiotic X-ray binaries [121]. In a similar vein, if newly-formed neutron stars are surrounded by a fallback accretion disk, early spin down via magnetic coupling may be another means of achieving rapid spin-down ([137]). However, it is not clear how common fallback accretion disks may be. Evidence for such disks is scant. There is evidence of an infrared excess indicative of a debris disk around one magnetar ([164]), and evidence of cold material being illuminated by a magnetar giant flare ([165]).

In sum, for both accreting and non-accreting neutron stars, the spin period can change rapidly enough that the current frequency is not a faithful representation of the initial state of the star. For accreting neutron stars, it is therefore also the case that the current spin axis of the neutron star should be strongly aligned with the binary orbital axis, as this determines the plane of accretion. As we now discuss, other more indirect approaches must be tried to determine the natal spins of neutron stars.

\subsection{Natal Neutron Star Spin}

As noted above, numerous mechanisms can drive spin frequency downward soon after a neutron star is formed. Over a much longer time scale, accretion in a binary system can drive spin frequency upward. This makes it difficult to estimate natal neutron star spins. However, it is important to attempt such estimates because several aspects of massive stellar evolution and progenitor events can only be resolved observationally; theoretical treatments leave large uncertainties. Understanding the natal spins of neutron stars provides a rare window into the spins of progenitor cores (e.g. [166],[167]). Similarly, the mechanisms that give rise to the (high) observed space velocities of neutron stars can be constrained by understanding their natal spin and magnetic fields (e.g. [168]).

A closer look at Table 1 of [130] reveals that there are a few rotationpowered pulsars for which the characteristic age $T=P / 2 \dot{P}$ is much greater than the age of the corresponding supernova remnant. Possible explanations for this discrepancy include misidentification of the correct remnant, a magnetic moment that is reduced on an improbably short time $<10^{5}$ years, or an initial pulsar period close to the current one. A different, and likely more reliable, way to constrain the initial period is to note that the spindown energy is ultimately delivered to the surrounding nebula in the form of high-energy charged particles. Given the reasonable assumption that the synchrotron cooling time is large, this means that the synchrotron luminosity 
of a supernova remnant gives clues to the initial spin period of the pulsar; if the period was short, the luminosity will be high.

Characterization of the observed distribution of neutron star spins using Monte Carlo studies that include various physical effects that alter spin can, in principle, provide an indirect guide to the distribution of natal spin values. The difficulty lies in characterizing the mechanisms that affect spin and stellar evolution. For instance, some observations suggest that neutron star magnetic fields can decay on short time scales (e.g. less than 5 Myr; [169, 170, 171]), although this is inconsistent with nuclear theory [172, 173] and other statistical studies find decay times $>100 \mathrm{Myr}[174,175,176]$.

The issue of "injection" also illustrates the difficulty with using the current neutron star spin distribution to understand the natal distribution. In order to explain the flow of pulsars in the period versus period derivative plane, a population of neutron stars with slow birth spin periods of $\sim 0.5 \mathrm{~s}$ was postulated [177], in addition to the population of Crab-like pulsars with shorter natal spins (100 ms or less). For many years, it was uncertain whether or not the injection of slowly-rotating pulsars was really required by the data, and concerns also arose regarding selection effects in the surveys from which the data were obtained. However, the balance of current opinion seems to be that "injection" is not required. Enforcing a luminosity cutoff that still provides for significant statistics, Lorimer found no evidence of injection [178]. A more recent analysis, using data from the Parkes Multibeam pulsar survey [179], finds evidence that many pulsars (40\%) may be born with periods of 0.1-0.5 s, but found no evidence for distinct sub-populations [180].

Secure estimates of natal neutron star spins require that the magnetic braking index be measured, and estimates are greatly aided when the age of an associated supernova remnant is known precisely. For these reasons, the Crab pulsar was the first to have its natal period estimated $\left(P_{0} \simeq 19 \mathrm{~ms}\right.$, [131]). A conservative (and likely robust) list of nine natal neutron star spin estimates is given in Table 7 of Faucher-Giguere \& Kaspi ([181]). The same authors also report Monte Carlo modeling of the observed pulsar spin distribution, finding that the distribution is normal, centered at $P_{0}=300 \mathrm{~ms}$, with a standard deviation of $150 \mathrm{~ms}$; this is broadly consistent with more recent studies [130, 182, 183], and evidence for injection is murky at best. Thus some pulsars could be born with few-ms periods, but most appear to start their lives rotating moderately. 


\subsection{Measuring Black Hole Spin with the Disk}

As we just discussed, the spin of neutron stars can be measured directly in pulsars. The fact of a stellar surface also means that a surface redshift can (in principle) be measured directly, for instance via redshifted absorption lines from the neutron star surface ([184], but see [185] ). Size measures are possible via several means, including thermal continuum emission from the stellar surface, though continuum emission is always subject to systematic effects including scattering in the stellar atmosphere, and magnetic confinement of the observed continuum to a region smaller than the full surface area.

In contrast, measurements of black hole spin are necessarily indirect. All current methods (and those potentially available in the near future with the exception of those involving gravitational waves; see Section 4) employ the accretion disk to infer the spin of the black hole. The two most commonly used methods involve fits to the $\mathrm{Fe} \mathrm{K} \alpha$ line profile or to the continuum spectrum. Both methods assume that the disk is geometrically thin and radiatively efficient, and that the fitted emission essentially terminates inside the innermost stable circular orbit (ISCO; see the discussion in Section 1). The continuum method also assumes that the black hole spin axis is aligned with the orbital axis of the host binary. We now briefly discuss these assumptions.

\subsubsection{Geometrically thin, radiatively efficient disks with an ISCO}

The classic studies of [186, 187] derived the structure of geometrically thin and radiatively efficient disks, with the additional assumption that there is some inner cutoff radius $R_{\text {in }}$ to the disk at which there is zero stress. With these assumptions, one might suppose that energy radiated in an annulus between radii $r$ and $r+d r$ is just the gravitational energy released there: $d E=G M m d r / r^{2}$, where $M$ is the mass of the central object and $m$ is the mass in the annulus. In fact, as first realized by Kip Thorne (see the footnote on page 344 of [186] ), there is an extra factor of 3 that enters for annuli well outside the inner edge because the transport of angular momentum from inner to outer annuli also transports energy. With an assumed sharp cutoff at $R_{\text {in }}$, the luminosity of an annulus becomes $\left(3 G M \dot{M} d r / r^{2}\right)\left[1-\left(R_{\text {in }} / r\right)^{1 / 2}\right]$ for an accretion rate $\dot{M}$, which integrates to $G M \dot{M} / R_{\text {in }}$ over the whole disk. If $R_{\text {in }}$ is the radius of the ISCO, then it has a significant dependence on the spin of the black hole, as shown in Figure 1,

Observations can partially address the question of whether $R_{\text {in }}=R_{\mathrm{ISCO}}$ for black hole accretion disks. Such studies must be limited to radiatively efficient, geometrically thin accretion disks. Recent observations with CCD 
spectrometers suggest that radiative efficiency is likely to apply for $L / L_{\mathrm{Edd}} \geq$ $10^{-3}$ (e.g. [188, 189], [190], [191]); an older, more conservative, and theorybased guideline might be $L / L_{\mathrm{Edd}} \geq 10^{-2}([192])$. The requirement of geometrical thinness imposes an upper limit of $L / L_{\text {Edd }} \lesssim 0.3$, depending on the application. Some studies of disks over a span of relatively high Eddington fractions, and over long periods of time, strongly suggest that inner disk radii are stable ([193], [194]). If the innermost edge of the disk were to fluctuate across the ISCO in response to variations in the mass accretion rate, the data would reflect this. Instead, the emitting area appears to be fairly constant at high Eddington fractions, and this suggests that disks are truncated at a fixed characteristic radius, which is likely to be at or near the ISCO. There is also evidence in some accreting neutron stars for the predicted effect of the ISCO on kilohertz quasi-periodic brightness oscillations [195, 196, 197, 198, 199], although the indirectness of the evidence has led to some cautionary notes by other authors [200].

Theoretical arguments and simulations can also bear on this problem. As matter within the ISCO plunges inward, it accelerates and becomes more ionized, and the emission is expected to be optically thin ([201]). This stands in strong contrast to direct and reflected emission from the accretion disk outside of the ISCO, which is expected to be distinctive and prominent. Recently, several numerical simulations have been attempted with the goal of addressing this question. There are essentially two complementary approaches. The first is to model the innermost disk region in the greatest detail possible, performing general relativistic magnetohydrodynamic (GRMHD) simulations in as many dimensions as is feasible. Such high-resolution simulations are critical to understand MHD effects near the ISCO [202, 203, 204, 205]. The second is to compromise on some detail but attempt to simulate for a large number of dynamical times in order to understand the long-term behavior of the disk in an equilibrium state. These simulations are needed to establish inflow equilibrium at larger radii. The most recent of the latter family of simulations strongly suggest that accretion disks respect the ISCO, and thus that direct and reflected emission from the disk can be used to infer the spin of the black hole within the ISCO [206, 207], although there remains some concern that these simulations might not have the resolution required to simulate the nonlinear turbulence properly.

There are some cautionary remarks to apply to these disk models. A natural first assumption is that each annulus emits as a blackbody. Indeed, such multitemperature models fit the data quite well [208]. However, more care- 
ful treatment of scattering and absorption processes lead to the theoretical conclusion that although the local spectra are close to a Planck function, the color temperature $T_{\text {col }}$ (which is the temperature one gets by fitting the data to a Planck function) is typically larger than the effective temperature $T_{\text {eff }}$ (defined such that the local flux is $\sigma_{\mathrm{SB}} T_{\mathrm{eff}}^{4}$, where $\sigma_{\mathrm{SB}}$ is the Stefan-Boltzmann constant) by a hardening factor $f_{\text {col }}=T_{\text {col }} / T_{\text {eff }} \approx 1.5-1.7$ [209]. The more careful models may indeed be correct, but all that can be said from the data is that the the emitting material is optically thick and has a characteristic emitting temperature near $\sim 1 \mathrm{keV}$ for stellar-mass systems. Whether the emission has the expected hardening factor of $\approx 1.5-1.7$ or whether it is locally blackbody does have implications for the implied spin, and because the observations alone cannot speak to the hardening factor the spins inferred using this method have some potential systematic errors.

A second issue is that recent observations of quasars with foreground microlensing have revealed discrepancies with the standard geometrically thin disk models [210, 211, 212, 213, 214, 215, 216, 217]. This has led to suggestions that, for example, the temperature might vary along a given annulus [218, 219, 220]. If there are similar deviations from the standard model in disks around stellar-mass black holes, this could affect spin determinations.

A third issue is that because radiation-dominated patches of accretion disks in MHD are apparently thermally unstable [221], this may limit the reliability of past simulations for determining what happens at high luminosities where radiation pressure dominates, and therefore for assessing possible systematic errors in black hole spin measurements.

Finally, most analyses of black hole spins do not take into account that the disks have finite thickness [206], which means that significant emission can persist inside the ISCO by roughly the disk height. This is especially important for rapidly rotating holes, for which the distance between the ISCO and the horizon need not be much larger than the disk thickness.

\subsubsection{Black hole spin axis orientation}

As we describe below, spin estimates using iron lines determine the angle between the spin axis and our line of sight as part of their fits. In contrast, degeneracies in the continuum models make it difficult to obtain robust fits for the disk inclination and black hole spin simultaneously [222]. This means that practical applications of the method require the disk inclination to be constrained by other data. A plausible assumption is that when the hole accretes from a companion, its axis is reoriented to align with the orbital 
axis. This, however, is not guaranteed. As we discussed in Section 1, the gas in the disk is torqued by frame-dragging induced by the black hole's spin, and thus in turn torques the black hole. The relevant radius in the disk is hundreds to thousands of gravitational radii, which means that the hole will be largely aligned by the time that a gas mass that is a fraction of $\sim$ few percent of the hole's mass has flowed over. For a canonical black hole mass of $10 M_{\odot}$ this means that a few tenths of a solar mass must have accreted. This is comparable to the mass of a typical low-mass companion, so it is quite possible that in many systems the required accretion has not happened [223, 224].

The data are ambiguous on this issue. Studies of some black holes conclude that the alignment is good, such as for XTE J1550-564 [225], whereas for other sources such as GRO J1655-40 (see [226] for the jet orientation and [106] for the binary orientation) and V4641 Sgr [227], significant misalignment has been inferred. Interestingly, it has been suggested that a misaligned inner disk could, at high accretion rates, rotate like a solid body and thereby produce the few-Hz quasi-periodic brightness oscillations seen in several accreting stellar-mass black holes [228, 83, 229, 230]. If this is accurate, it means that spin axes can be somewhat independent of orbital axes.

With this background, we now discuss the two major methods for inferring the spins of stellar-mass black holes.

\subsection{Relativistic Disk Reflection}

Dense gas can scatter or "reflect" incident X-ray emission ([231], [232]). This phenomenon is observed in numerous physical contexts. For instance, particular clouds of gas in the Galactic Center are observed to have strong Fe K emission lines, likely stimulated by ancient X-ray emission from Sgr A* (e.g. [233]). Strong, narrow Fe K emission lines are also observed in the spectra of numerous Seyfert-1 and Seyfert-2 AGN, owing to hard X-ray irradiation of the torus and/or broad line region (e.g. [234], [235]; also see [236]). Indeed, in some Seyfert-2 spectra, emission from the central engine is almost entirely blocked from direct view, and the observed spectrum is strongly dominated by reflected light.

The most prominent part of typical reflection spectra is Fe K line emission, owing to the abundance and fluorescence yield of iron, and also to the ability of He-like and H-like charge states of iron to retain electrons at very high temperatures and ionization parameters (e.g. [232]). The other important features of a reflection spectrum include photoelectric absorption from the Fe 
$\mathrm{K}$ shell (which produces an apparent flux decrement), followed by a recovery of the continuum peaking at about $30 \mathrm{keV}$ (this is where the albedo of the disk peaks), and a gradual flux decline at higher energy (the albedo is reduced as incident photons penetrate deeply into the gas and do not readily reemerge). Depending on the specific nature of the gas and the intensity of the irradiating flux, Fe L lines can also be quite prominent ([237]. Figure 2 shows the relativistic line and reflection spectrum in a recent NuSTAR spectrum of GRS 1915+105 [238]. Careful construction of the reflection models is critical for all spin determinations using this method [237, 239, 240, 241, 242].

An accretion disk will intercept a large fraction of any hard X-ray flux generated in the accretion process. Ignoring potentially important considerations such as gravitational light bending [243, 244, 245, 246] and light travel times, the disk should subtend approximately $2 \pi$ steradians as seen from a source of hard X-ray emission. Utilizing the disk reflection spectrum requires a change of reference frame. Reflection spectra are calculated in the fluid frame of the disk - all of the lines and features are intrinsically narrow. At infinity, however, an observer sees a spectrum that has been "blurred" by relativistic Doppler shifts and gravitational red-shifts. Because the ISCO becomes smaller with higher spin parameters, the degree of "blurring" is set by the spin. Thus with a simple relativistic smearing model (usually just a library of rays traced from close to the black hole to infinity; see [247, 248, 249, 250, 251, 252]) the spin can be measured in sensitive X-ray spectra (see, e.g., [253]).

In practice, when fitting a blurred disk reflection spectrum to measure a spin parameter, a few crucial variables must be measured in addition to the spin. Parameters central to the blurring include the emissivity of the disk reflection spectrum (typically a power-law in radius, with zero-two breaks; see $[253,254,255]$ ), and the inclination of the inner disk. If the nature of the corona is known a priori, the emissivity function can be fixed, but this is not a requirement for a spin measurement. In the limit of less sensitive spectra, it is often expedient to assume that a resolved relativistic jet axis traces the black hole spin axis, and that this vector is normal to the inner accretion disk (as expected owing to gravito-magneto-hydrodynamic effects; e.g. [53, 71]). Parameters central to characterizing the reflection include the ionization of the disk, and the flux normalization of the reflection spectrum. In some cases, it may also be necessary to measure or set the metal abundances of the reflector (see [254]), though strong abundance anomalies may be artifacts 


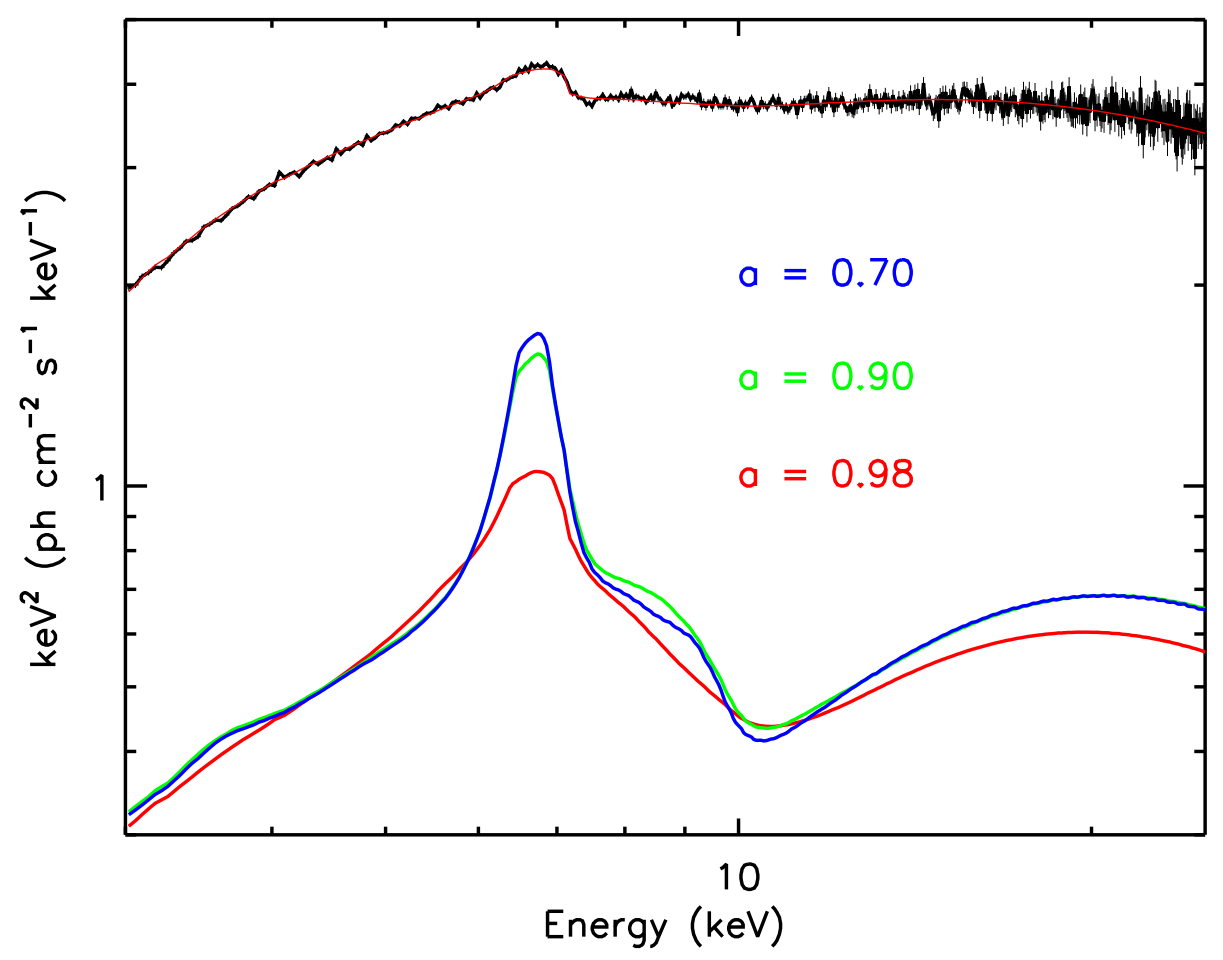

Figure 3: This figure shows a NuSTAR spectrum of GRS 1915+105 (in black), fit with a disk reflection model (in red) that gives a spin of $a=0.98 \pm 0.01$ [238]. The contribution of the best-fit disk reflection spectrum to the total spectrum is shown below the data, in red. To illustrate the effect of black hole spin on the reflection spectrum, we show models with spins of $a=0.90$ and $a=0.70$ in green and blue, respectively. The effect of spin is clearly to broaden the reflection spectrum, and to give a stronger red wing to the $\mathrm{Fe}$ $\mathrm{K}$ line profile. Indeed, the power of this technique for spin measurements lies partly in the fact that it is a relative measurement. Note that the data must also constrain other parameters that are important in shaping the disk reflection spectrum, including the inner disk inclination and emissivity, in order to determine the spin of the black hole.

of an effect similar to "magnetic levitation" observed in the sun.

Several reviews of disk reflection and related topics have been published (see, e.g., [10, 256, 253]). The level of detail in those reviews exceeds what is possible to provide in this spin-centered treatment, and the reader is referred to the prior work for additional nuances of disk reflection spectroscopy. For 
this review, we simply proceed to briefly comment on the primary features of this technique, and to highlight some key results.

This spin measurement technique has some notable strengths and advantages:

- It relies primarily upon lines and atomic physics, and relative measurements (e.g. line width) rather than absolute measurements (e.g. absolute fluxes or absolute magnitudes). Traditionally, astrophysics is a field that excels at relative measurement, but faces numerous challenges with absolute measurement because it is mostly an observational science, not an empirical one.

- Similarly, relativistic distortions to the reflection spectrum depend on the relative depth of the inner disk within the potential well - things scale in a $G M / c^{2}$ sense, obviating the need to know the mass of the black hole, the distance to the source, or the accretion rate a priori. Spin measurements have been obtained from (clear, convincing) black hole candidates whose mass functions have not been measured (e.g. [257, 258, 259]).

- Disk reflection occurs in both stellar-mass black hole binaries, and in AGN. This enables tests and explorations of black hole spin across the mass scale. This is likely to be very important for investigations of the role that black hole spin may play in powering relativistic jets (e.g. [260]).

- Reflection spectra are also sensitive to the inclination of the inner disk, which need not be the same as the inclination of the outer disk or binary system $([223])$. Reflection can then serve to obtain a unique measure of the inner accretion disk inclination.

- In principle, the geometry of the hard X-ray corona can be inferred through the disk reflection emissivity. For example, if the observed iron line comes only from a disk illuminated by an isotropic source of hard X-ray photons, the radial dependence of the emissivity will be $J \propto r^{-3}$, but it is not certain that this is the correct emissivity distribution [261, 262]. For instance, if the source of hard photons is above the disk plane on the disk axis, gravitational light bending can produce a significantly stronger radial dependence of the illumination on the disk. Thus the emissivity can encode how central or how extended the corona is, what its height above the disk may be, and whether 
or not gravitational light bending is important ([255], also see [263]). The emissivity function is often captured as a power-law in radius, with zero, one, or two breaks. From the observational standpoint, microlensing data for quasars suggest that the X-ray source size is much smaller than that of the optical or UV [264, 213], which might imply that the emissivity index is steeper than $r^{-3}$; this would be compatible with the steep indices required in some disk reflection fits of AGN (e.g., [265] and subsequent papers). In addition, it is important to note that the decrease in surface density across the ISCO region is likely to create a cutoff in emissivity, but exactly where it happens depends on details. Thus the shape of this cutoff and exactly how it is positioned with respect to the ISCO introduce a certain amount of systematic uncertainty in the spin determination [266, 267, 261]. Recent work [268] suggests that the uncertainty might be $\sim \Delta \hat{a} \sim 0.2$, which is comparable to or less than uncertainties in distance or inclination.

Examples of measurements via disk reflection (see Table 1 for a full list):

GRS 1915 $+\mathbf{1 0 5}$ is one of the best-known stellar-mass black holes, largely because of its prodigious jet activity. In some phases, it produces steady, compact jets; in other states, it has been observed to launch discrete "cannonballs" with apparent speeds in excess of $c([269])$. This activity makes GRS $1915+105$ a particularly interesting source in which to try measure the black hole spin, since spin may power jets $([270])$. Early efforts at measuring the spin via reflection were complicated by the detector limitations, but indicated a high spin consistent with $\hat{a}=0.9$ ([271]; also see [272]). Later efforts to measure the spin using a deep Suzaku observation were also inconclusive ([273] ). A new observation with $N u S T A R([274])$, which offers a fertile combination of spectral resolution, sensitivity, and band pass, has revealed an excellent blurred disk reflection spectrum and gives $\hat{a}=0.98 \pm 0.01$ ([238]) . This spin value is broadly consistent with one measurement obtained using the disk continuum ([275]). However, for such high spins the finite thickness of the disk will likely soften the limit to $\gtrsim 0.9$. Put more strongly, the assumption of a razor-thin disk that goes into the spectral modeling must break down at the ISCO of a $\hat{a}=0.98$ black hole as close to Eddington as GRS $1915+105$.

Some efforts have attempted to move beyond measuring spins in black holes. Variability trends between the flux incident upon the disk, and the reflection spectrum, can potentially serve as an indication of gravitational 
light bending (e.g. [243]). Essentially, in a simple Euclidean scenario, the reflected flux should track the incident flux in a linear fashion. In practice, the two are positively correlated for a range of incident flux levels, but thereafter the reflection spectrum does not respond. This is consistent with a central corona collapsing to small scale heights, such that most of the hard X-ray emission is focused onto the disk rather than emitted isotropically ([243, 244, 245, 246]). Light bending is expected if hard X-ray emission is generated close to spinning black holes, so the potential detection of light bending serves as a potential confirmation of the method and measurements.

XTE J1650-500 is a black hole for which spin has been measured quite well, and its strong disk reflection spectrum supplies the best evidence of gravitational light bending. Arguably, the first reflection-derived spin from a stellar-mass black hole was obtained in XTE J1650-500: a blurred iron line detected with XMM-Newton and an inner disk radius consistent with $r=1.24 G M / c^{2}$ was measured ([276] ), indicating a near-maximal spin. Later modeling using more advanced disk reflection spectra found a spin of $\hat{a}=0.79 \pm 0.01([257])$. More intensive monitoring with BeppoSAX and RXTE found a non-linear relationship between the direct and reflected flux, consistent with the relationship expected if flux is partly modulated by gravitational light bending close to a spinning black hole $([244,245])$. New work has eliminated the possibility that the flux trend is driven by the ionization of the disk, and affirmed the signature of light bending ([246]).

GX 339-4 is perhaps thee best-known stellar-mass black hole that is a recurrent transient. The best measurements of black hole spin in GX 339-4 have been obtained in elevated flux states, e.g. "intermediate" and "very high" states using XMM-Newton and Suzaku. Fits to a very skewed iron line and reflection spectrum obtained with XMM-Newton during the 2002-2003 outburst of GX 339-4 measured inner disk radii in the $2-3 G M / c^{2}$ range ([277]), corresponding to spin values of $a \simeq 0.8-0.9$. Later observations made using Suzaku during the 2006-2007 outburst of GX 339-4 measured a spin of $a=0.89 \pm 0.04$ ([278]). Joint fits to the XMM-Newton and Suzaku data give a spin of $0.93 \pm 0.04([278])$. Owing to its strong, skewed reflection spectrum, new reflection models have been tested against data from GX 339-4, enabling one measure of the modeling-based systematic errors associated with reflection-derived spins. Related errors appear to be small, at the level of a few percent or less $([279])$.

XTE J1752-223 is a black hole candidate in which the spin has been measured well, and like XTE J1650-500 it extends the reach of spin measure- 
ments and reflection. Using $X M M-N e w t o n$ and Suzaku, disk reflection spectra from this black hole candidate were measured, and consistency between the "intermediate" and "low/hard" states was found ([259]). This is significant in that the disk may be radially truncated in the low/hard state, but - at least in these sensitive spectra - the disk instead appears to remain at the ISCO. Fitting the disk reflection spectra jointly, a spin of $\hat{a}=0.52 \pm 0.11$ was measured ([259]). This moderate value likely indicates that reflection-based measures of black hole spin are not artificially biased toward high values.

The success of disk reflection in measuring black hole spins has spawned efforts to harness the same technique in accreting neutron star X-ray binaries (e.g. [280, 281, 282]). In principle, the extent of relativistic blurring would allow the dimensionless spin of the neutron star to be measured. However, this method is likely to be less practical than timing techniques. The more interesting constraint that may be possible via disk reflection in neutron stars is a constraint on the size of the neutron star, in cases where the stellar mass is known. Simply, the stellar radius must be less than the radius of the inner disk. Converting an inner radius measured via disk reflection into physical units from gravitational radii then provides an upper limit on the neutron star radius. Currently, the most convincing relativistic line profile and best data are from an observation of Serpens X-1 with $N u S T A R$, which give $r_{N S} \leq 15 \mathrm{~km}$ (and thus a surface redshift $z_{N S} \geq 0.16$ for $M_{N S}=1.4 M_{\odot}$ ) for a broad set of fits, and $r_{N S} \leq 12.6 \mathrm{~km}$ if the disk extends to the ISCO $([283])$. Incidentally, although the spacetime around a rotating object is unique to first order in rotation [284], to higher orders the structure depends on the detailed nature of the object. In particular, the Kerr spacetime is not a good approximation for the external spacetime of a rapidly rotating neutron star [285], so for such objects numerical spacetimes must be used.

\subsection{The Accretion Disk Continuum}

It is also possible to measure the spin of a black hole using the thermal continuum emission from the accretion disk [286, 11]. A number of results suggest that accretion disks in the "high/soft" state are well described in terms of a standard "thin" accretion disk ([186]). This means that the emission vanishes at the ISCO as there is zero torque, peaks at a radius just outside of the ISCO, and follows a $T \propto r^{-3 / 4}$ profile far from the ISCO. Essentially, then, the disk can be modeled as a set of annuli; this is easily encoded into spectral fitting packages (see, e.g., [208]). 
Any blackbody flux nominally gives a ratio of the observed emitting area over the line-of-sight distance, $r^{2} / d^{2}$, modified by the inclination angle. The mass of the black hole must also be known, in order to translate the physical radii into gravitational radii, and thus determine the spin. Thus, to employ continuum fitting, the mass and distance to the black hole must be known a priori and independently. Owing to the fact that thermal emission is a continuum and does not bear the imprints of Doppler shifts as distinctively as atomic features, the inclination angle of the inner accretion disk (which may differ from the binary inclination; [223]) must also be known a priori. Last, radiative transfer through a disk atmosphere will modify the spectrum emitted in the midplane. As we discussed above, the observed spectrum must be corrected by a hardening factor in order to measure a spin. A value of $f=1.7$ is taken to be canonical ([287]), though a broader range is possible depending on the nature of the corona (e.g. [288]). New models for accretion disks capture all of these parameters and are able to turn these absolute photon flux measurements into black hole spins (e.g. [289], [209]). Figure 4 depicts the influence of spin on the accretion disk continuum.

Distances within the Galaxy are typically difficult to determine, and errors can be comparable to the distance value itself. Of course, carrying forward large errors into spin measurements should result in large uncertainties. This situation has recently improved drastically: VLBA astrometry has made it possible to determine parallax distances for some black holes (e.g. V404 Cyg and Cyg X-1; 290, 291]), reducing errors to a very small fraction of the distance value. Masses are also difficult to determine, typically giving very large fractional errors (see, e.g., [292]). Inner disk inclinations can be determined via relativistic jets, assuming that the jet axis is the spin axis and normal to the inner disk [225]. Setting the inner disk inclination to that of the binary system is a less robust assumption, but provides a path forward when other constraints are unavailable.

Like the disk reflection method, estimates of spin using the disk continuum have their own strengths:

- In soft spectral states, wherein the disk continuum may be employed to measure spins, the disk represents the bulk of the photon flux and the bulk of the energy output. When the mass, distance, and inclination are known precisely, this can translate into precise black hole spin measurements.

- Harnessing the bulk of the observed flux also means that spectra from stellar-mass sources in the Local Group, and potentially within galaxies at 


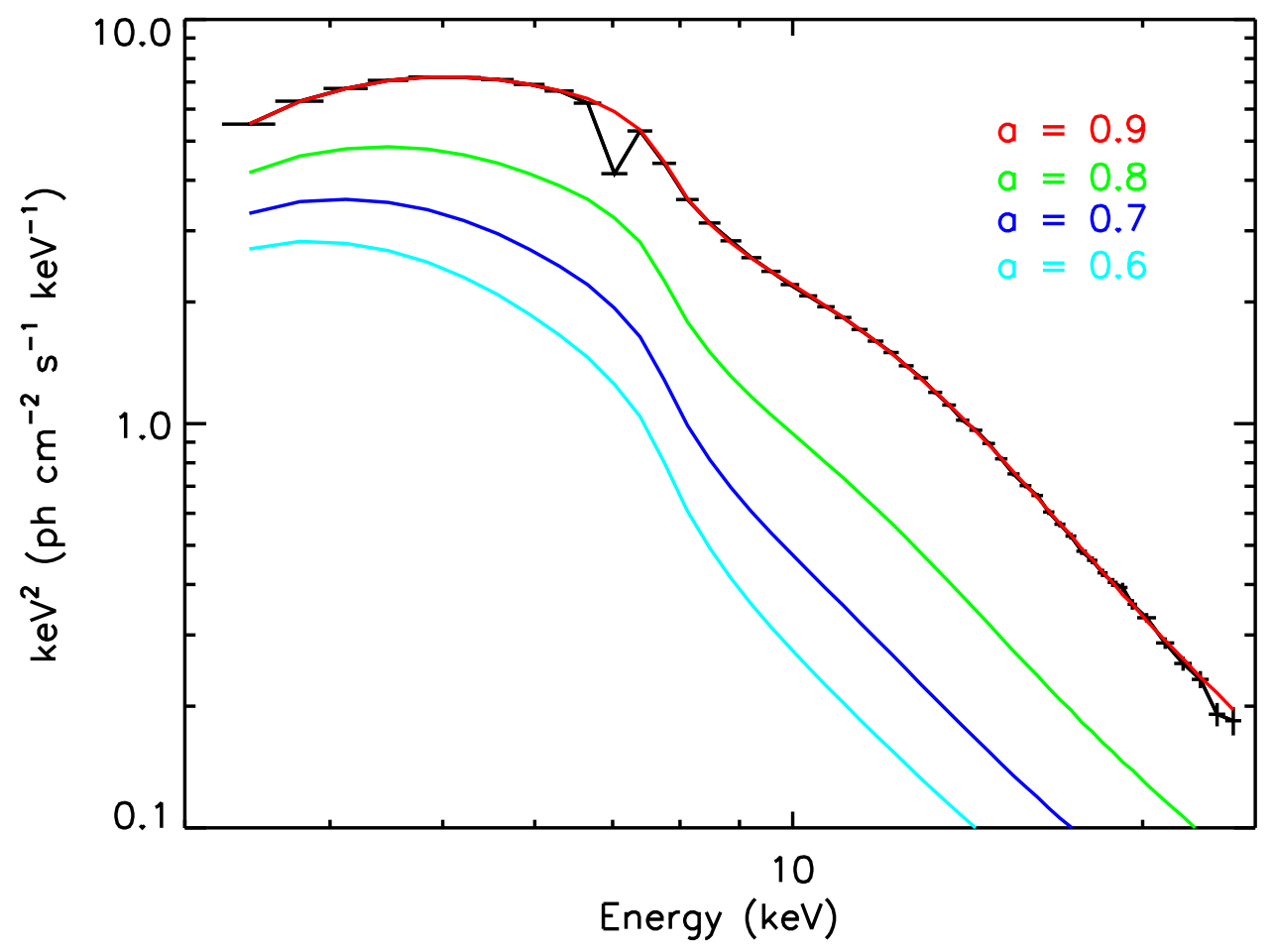

Figure 4: This figure shows an RXTE spectrum of GRS 1915+105 (in black), fit with a model that requires a spin of $a=0.9$ (in red). The spectrum is observation 4 within [275]; the fitting procedure outlined in that work was employed in this fit. The model also includes a hard component, and the choice of the hard component impacts spin values. The notch near to $7 \mathrm{keV}$ is an absorption line included in the model. A disk close to a spinning black hole emits more at all wavelengths; to illustrate the effect of spin, then, we show models assuming $a=0.8, a=0.7$, and $a=0.6$ in green, blue, and cyan, respectively. Continuum-based measurements of spin are able to utilize the bulk of the observed X-ray flux, but they are an absolute measurement and thus require the mass and distance of the black hole to be known precisely. It is also important to know the inner disk inclination and atmospheric scattering corrections from other observations or theoretical considerations. Note that actual fits with different spin parameters must allow the hard component to vary and adjust to optimize the fit, and that the data must constrain all model components in order to deliver robust spin measurements.

distances of a few Mpc, can be used to measure spins. In such cases, distances are surprisingly known with much better precision and accuracy than is gen- 
erally possible within the Milky Way; the prime difficulty for extra-galactic sources lies in measuring the black hole mass.

- Monitoring observations obtained with spectrometers that have low spectral resolution are often sufficient to characterize the disk continuum well. Therefore, numerous spectra can be fit together, and thus jointly determine a spin parameter. This may reduce the influence of fluctuations in other parameters in deriving a spin value.

Examples of measurements via the disk continuum (see Table 1 for a full list):

GRS $1915+105$ is measured well using the accretion disk continuum. It is not necessarily obvious that this should be the case, because the line-ofsight column density to GRS $1915+105$ is very high and scatters away most of the flux below $2 \mathrm{keV}$; however, the high disk temperature compensates and allows robust determinations based on the Wien tail of the distribution. Fairly accurate black hole masses and distances [293], and an inclination defined by extended radio jets [294], also contribute to good measurements. Based on fits to numerous spectra selected for a a flux strongly dominated by the disk and a low Eddington fraction, a spin of $\hat{a} \geq 0.98$ was measured [275]. It is noteworthy that the higher spin value agrees with the reflection measurement [238]. A spin estimate of $\hat{a} \simeq 0.7$ was also reported [295], but included observations where the source may have been close to Eddington, potentially inconsistent with the model assumption that the disk is geometrically thin.

The spin of Cygnus X-1 was recently measured well using the disk continuum [296] and later via reflection [297, 298], and commensurate values were again obtained. Employing a new distance derived using radio parallax measurements [291], and improved binary system parameters ([299]; however, see [300]), a spin of $\hat{a}>0.95$ is measured [296].

It is worth noting that reflection-based measurements of the spin of Cygnus X-1 have improved with better-suited detectors and read-out modes. Fits to $A S C A$ gas spectrometer data obtained in a high/soft state were consistent with a near-maximal spin, but were not able to strongly exclude lower values [271]. In contrast, spin estimates based on fits to spectra obtained in the low/hard state using the $X M M-N e w t o n / E P I C-p n$ CCD camera suggested a very low spin [257]. It is now clear that photon pile-up (which occurs when the rate of photon arrival is greater than can be processed by the instrumentation) can artificially narrow relativistic skewing [301], and the value 
obtained with XMM-Newton might best be regarded as a lower limit. The most recent value of $\hat{a}=0.97_{-0.02}^{+0.01}$ was obtained with Suzaku [297], employing a combination of CCD spectra at low energy and broad-band coverage up to $400 \mathrm{keV}$.

M33 $\mathrm{X}-\mathbf{7}$ provides an example of how continuum fitting may be applied to black holes in nearby galaxies. The distance to M33 is known well, and $\mathrm{X}-7$ happens to be an eclipsing source - immediately providing a strong inclination constraint and facilitating a mass constraint. With these advantages, a spin of $\hat{a}=0.84 \pm 0.05$ was measured [302, 303].

LMC X-3 is another interesting example of the continuum method. Following an extensive effort to re-calibrate the flux normalization of historical $\mathrm{X}$-ray missions relative to the Crab (now known to be slightly variable), it was possible to show that the inner radius of the disk in this source may be fairly constant, signaling conditions that would permit a spin measurement [194]. Binary system parameters can be particularly difficult to derive when the donor star is massive, however, so despite the compelling X-ray data, a spin measurement had to await improved system constraints [304]. A spin of $\hat{a}=0.21_{-0.22}^{+0.18}$ has now been obtained [305]. It is unclear whether it is significant that this is a smaller spin than has been obtained for other sources.

\subsection{Quasi-Periodic Brightness Oscillations}

In principle, quasi-periodic brightness oscillations (QPOs) provide a simple and precise means of measuring black hole spin. The highest frequency QPOs detected in stellar-mass black holes are broadly consistent with the orbital frequency at the ISCO, which is $\nu_{I S C O}=220 \mathrm{~Hz}\left(10 M_{\odot} / M_{B H}\right)$, for $\hat{a}=0$ and is larger for prograde spins. These features are narrow enough compared to their frequency that they could in principle provide very specific information. A hint as to the mechanism is that in a few systems QPOs are detected in roughly stable 3:2 frequency ratios (for a review, see [292]), although many models can get approximately this ratio (see the discussion in Section 5.2 of [306]). Note that there is some evidence that the frequencies can change by at least several percent [307, 308], which might be caused by the inner disk becoming marginally optically thin in some circumstances [309]), even in distinct outbursts separated by several years (XTE J1550-564 is one such case; see [310, 311]), suggesting that the frequencies are anchored by gravity and not fleeting manifestations of processes in an MHD disk that would reveal nothing of the black hole itself. 
The pragmatic difficulties with using QPOs to measure spin are many. Whereas nearly every stellar-mass black hole shows continuum emission from the disk, as well as disk reflection, only a minority show high frequency QPOs, and a smaller minority manifest QPOs in a 3:2 frequency ratio (292, 312]; note that GRS $1915+105$ has two such pairs, one pair at $67 \mathrm{~Hz}$ and $41 \mathrm{~Hz}$ and the other at $164 \mathrm{~Hz}$ and $113 \mathrm{~Hz}$ ). Moreover, high frequency QPOs are rare, sometimes only detected in a few observations out of many hundred sampling an outburst, or in the addition of small segments of hundreds of observations that satisfy flux and spectral hardness selection criteria (e.g. [310], [313]). Last, spins based on QPO frequencies can require the mass of the black hole to be known. These difficulties stand in marked contrast to the simplicity of disk reflection, for instance, which can measure a spin using a single sensitive spectrum, without knowledge of the black hole mass.

The physical difficulties with using QPOs to measure spin are as severe as the pragmatic hurdles. The energy spectrum of QPOs is decidedly not disklike (e.g. [310, 313]). The rms amplitude of QPOs increases with increasing energy. Indeed, the highest frequency yet detected $-450 \mathrm{~Hz}$ in power spectra of GRO J1655-40 - was only detected in the 13-27 keV band with RXTE (see [313]; a disk temperature of $k T \simeq 1 \mathrm{keV}$ is characteristic of a stellarmass black hole close to Eddington). If QPO frequencies are indeed disk frequencies, the disk must somehow transmit the frequency into a corona where it is expressed. Alternatively, it may be the case that QPO frequencies are not disk frequencies; some models predict QPOs produced at the coronal base of a jet [314].

Owing to the degeneracy of different QPO models, examining different interpretations and spin estimates for a single black hole is likely more practical than touring the different models in the abstract. The most interesting case is likely GRO J1655-40, because the $450 \mathrm{~Hz}$ oscillation detected from this source is the highest-frequency yet detected in a stellar-mass black hole (note that a $300 \mathrm{~Hz}$ oscillation is detected at the same time, though not in the same energy range; see [313]).

- If the $450 \mathrm{~Hz}$ oscillation represents the Keplerian orbital frequency at the ISCO, constraints on the mass range for GRO J1655-40 then constrain the spin to be $0.15 \leq \hat{a} \leq 0.5[313]$.

- If either the $300 \mathrm{~Hz}$ or $450 \mathrm{~Hz}$ oscillations are a Lense-Thirring precession frequency, then the black hole must be rotating at or near the maximum possible value.

- The oscillations may be resonances between Keplerian, periastron pre- 
cession, and nodal precession frequencies [315]. Such a resonance could account for the $300 \mathrm{~Hz}$ and $450 \mathrm{~Hz}$ oscillations and would give $0.4<a<0.6$, but the predicted nodal precession could not be associated with the observed $18 \mathrm{~Hz}$ QPO also detected in GRO J1655-40 [313]. More recently, a spin of $\hat{a}=0.290 \pm 0.003$ has been reported, assuming the QPOs arise via the relativistic precession mechanism [316]. Note, however, that this mechanism assumes geodesic motion, but eccentric or out-of-plane motion would be expected to be damped by fluid interactions.

- Normal modes of disk oscillations - diskoseismic modes - are another potential model for high-frequency QPOs [317, 318, 319, 320], although these have not been seen in magnetohydrodynamic simulations [306]. The fundamental $g$-mode is expected to be the most robust oscillation mode, and the most readily detectable. The $450 \mathrm{~Hz}$ oscillation of GRO J1655-40 cannot be associated with any $g$-mode. If the $300 \mathrm{~Hz}$ oscillation is associated with the fundamental $g$-mode, then a spin of $0.86<a<0.98$ is implied [321].

Data from observations of GRO J1655-40 and other stellar-mass black holes do not prefer any specific model for the observed frequencies. The same could be said for the few-Hz QPOs seen in many stellar-mass black hole candidates; these are stronger than the high-frequency QPOs, and are often sharper, so although their origin is not known with confidence, if they are linked to the mass and/or spin of the black hole they might ultimately be useful as probes. Therefore, although it is possible to measure black hole spin via QPOs in principle, the data do not yet allow for a reliable measurement. If future data (for instance, from Athena [322] or LOFT [323]) are able to identify a particular model as correct, then true measurements will become possible.

Current models for high frequency QPOs share some fundamental difficulties. The frequencies are explained within a model, but not the specific modulation mechanism. Nor do models explain the energy dependence of QPOs, or the nature of other facets such as phase lags. Depending on the specific model assumed when interpreting QPOs, these difficulties may be more or less problematic.

It is additionally unclear why some sources show QPOs in a 3:2 frequency ratio (e.g. GRO J1655-40, XTE J1550-564, XTE J1650-500, H 1743-322; [313, 311, 324, 325]), while others appear to only show a single QPO (4U 1630-472, [326]), and many sources show no high frequency QPOs (e.g. 4U 1543-475, GX 339-4, XTE J1817-330).

As was discussed by [327], it is remarkable that the sources wherein high 
frequency QPOs have been detected are all observed at high inclinations $\left(i \geq 50^{\circ}\right)$, at least if the inclination of the inner disk is assumed to be that of the binary. In contrast, the sources wherein high-frequency QPOs have not been detected are likely viewed at much lower, face-on inclinations. This raises intriguing possibilities:

- First, it may be the case that QPOs are more easily detected at high inclinations owing to special relativistic beaming effects associated with the orbits and/or resonances that produce the oscillations.

- Second, if the oscillation is produced via any sort of precession or oscillation in a vertical direction, an inclination angle closer to the plane of the disk may be advantageous. Special relativistic boosting may also aid detection from high inclination angles, in such a scenario.

- Third, it may be that sources viewed at low inclinations produce less Comptonized radiation, and that the scattering process is somehow important to the production of high frequency QPOs. The energy dependence of QPOs may suggest this independently. This might be true if scattering through the disk atmosphere is not a simple constant, as envisioned in the application of simple multiplicative $f_{\text {col }}$ factors. Naively, the path length through an atmosphere to an observer at high inclination should be longer. Potentially, then, $f_{\text {col }}$ may need to be written as $f_{\text {col }}(i)$ or $f_{\text {col }, 0}+f_{\text {col }}(i)$, where $i$ is the inclination angle, and $f_{\mathrm{col}, 0}$ might be unity or the traditional value of $f_{\text {col }}=1.7$. This would have obvious consequences for spin measurements made using fits to the accretion disk continuum that have assumed $f_{\text {col }}=1.7$ for all inclinations.

Last, the recent outburst of the black hole IGR J17091-3624 poses some interesting questions about the use of QPOs to study black hole spin. Detections of the the $188 \mathrm{~Hz}$ and $276 \mathrm{~Hz}$ QPOs in distinct outbursts of XTE J1550-564 strongly signals that the observed frequencies are anchored by gravity, and not subject to MHD nuances or $\dot{M}$. However, $66 \mathrm{~Hz}$ QPOs are detected in IGR J17091-3624 [328], remarkably similar in frequency and other properties to the famous $67 \mathrm{~Hz}$ QPOs detected in GRS 1915+105 [329]. It is entirely possible that this is simply coincidental. However, it raises the prospect that these oscillations are physically distinct from the higher frequency oscillations detected in GRO J1655-40 and XTE J1550-564, in which case some of the QPOs might not be anchored by gravity. 


\subsection{Spin from X-ray Polarization}

Currently, it is not possible to measure polarization in the soft X-ray band, where accretion disks dominate the spectrum of stellar-mass black holes. However, considerable theoretical efforts have been undertaken to develop spin diagnostics based on polarization. In particular, the closer an accretion disk extends to the black hole owing to black hole spin, the greater the fraction of "returning radiation", which is radiation that is gravitationally lensed back to the disk and which is polarized orthogonal to radiation emitted by the disk far from the black hole ([330]; see also [331]). Thus spin has an energy-dependent effect on both the degree of polarization and the observed polarization angle.

Because soft X-ray polarization has not yet been measured it is not clear how much of the non-idealities of disk and jet emission that we discussed earlier will cloud inferences about the spin magnitude and direction. There is, however, plausible optimism that polarization will at least provide complementary measurements, and in the best case it might be free of some of the systematic issues of current spin estimations. For some references to the possibility of polarization measurements constraining spin and inclination, see [332, 333]. There are also some promising suggestions that polarization observations may be able to constrain coronal properties [334] and that, at least in currently explored models, such measurements in the thermal disk state require no a priori knowledge of the spin, inclination, mass, or distance ([330], although the previous caveats about disk physics complexity must be kept in mind). Proposed future polarization missions include $X$-Calibur [335, 336] and GEMS [337, 338].

\subsection{Theoretical Expectations for Spins}

Massive black holes can grow in mass and spin through sustained disk accretion. However, if accretion is chaotic - if some accretion episodes precipitate a retrograde disk - then accretion onto massive black holes may not drive them to high spin parameters. Moreover, massive black holes can grow through mergers with other black holes; successive additions of random spin vectors will tend to spin-down the resultant black hole (see, e.g., [339]). In Seyfert AGN, at least, the observed distribution of black hole spins is skewed to high, positive values, and favors black hole growth through periods of sustained disk accretion. Spin measurements are even more sparse among quasars, but early measurements again point to high spin values and sustained disk accretion as an important mode of mass growth [340, 341, 342]. 
The situation is markedly different for stellar-mass black holes. As we discussed earlier, the spin of stellar-mass black holes is largely determined at birth. Thus numerous factors may be important in predicting the spin distribution of stellar-mass black holes, including the mass of the progenitor star, its spin rate, and its interior structure. In particular, different outcomes may result from a very massive star where magnetic fields have worked to produce nearly solid-body rotation, versus a scenario where differential rotation is still possible. This problem has been examined in the context of the means by which massive black holes may arise in galactic centers: the collapse of a single super-massive star. This situation may not be directly analogous to the collapse of a normal star. However, some models suggest that $\hat{a} \sim 0.7$ could typically emerge from core collapse, in contrast to the spin of $\hat{a} \sim 0.9$ that is predicted over long times due to accretion, as in supermassive black holes [51].

Recently, the distribution of spin parameters in stellar-mass black holes has been examined with two purposes: to test the predictions of models, and to compare that distribution to natal spins inferred for neutron stars as a window into their birth events. The mean spin measured via reflection was found to be $\hat{a}=0.66$, and the mean spin measured via continuum fitting was found to be $\hat{a}=0.72$ [167]. Not only are these mean values in good agreement, but so too are the distributions. Although there are isolated cases of discrepant measurements, it is likely that both methods are making solid physical measurements. Moreover, these spin values differ markedly from natal spins inferred from neutron stars, and suggest that black holes may preferentially form in SNe events with enough angular momentum to drive strong MHD jets, causing them to appear as GRBs [167].

As we see from Figure 5, the currently known angular momenta of black holes are at least two orders of magnitude larger than the angular momenta of neutron stars, even those spinning within a factor of 2-3 of breakup. This strongly suggests that black holes acquire vastly larger angular momenta at birth than do neutron stars. Fallback from the pre-supernova star is one clear possibility for the source of this extra angular momentum.

\section{Gravitational Waves}

The era of direct gravitational wave detections is expected to commence in a few years, when second-generation ground-based detectors such as Advanced LIGO [360], Advanced Virgo [361], KAGRA [362], and LIGO-India 


\begin{tabular}{llll} 
Object (by RA) & Disk Reflection & Disk Continuum & References \\
\hline M33 X-7 & & $a=0.84 \pm 0.05$ & {$[302,303]$} \\
LMC X-3 & & $0.21_{-0.22}^{+0.18}$ & {$[222,305]$} \\
LMC X-1 & $0.97_{-0.13}^{+0.02}$ & $0.92_{-0.07}^{+0.05}$ & {$[343,344]$} \\
A 0620-00 & & $0.12_{-0.19}^{+0.19}$ & {$[345]$} \\
GS 1124-683 & & $-0.24_{-0.64}^{+0.05}$ & {$[346]$} \\
4U 1543-475 & $0.3 \pm 0.1$ & $0.8 \pm 0.1$ & {$[257],[347]$} \\
XTE J1550-654 & $0.55 \pm 0.22$ & $0.34_{-0.45}^{+0.37}$ & {$[257,348]$} \\
4U 1630-472 & $0.985_{-0.014}^{+0.005}$ & & {$[349]$} \\
XTE J1650-500 & $0.79 \pm 0.01$ & & {$[276,257]$} \\
XTE J1652-453 & $0.45 \pm 0.02$ & & {$[258]$} \\
GRO J1655-40 & $0.98 \pm 0.01$ & $0.7 \pm 0.1$ & {$[257,347]$} \\
GX 339-4 & $0.94 \pm 0.02$ & & {$[257]$} \\
SAX J1711.6-3608 & $0.6_{-0.4}^{+0.2}$ & & {$[257]$} \\
XTE J1752-223 & $0.52 \pm 0.11$ & & {$[259]$} \\
Swift J1753.5-0127 & $0.76_{-0.15}^{+0.11}$ & & {$[350]$} \\
MAXI J1836-194 & $0.88 \pm 0.03$ & & {$[351]$} \\
XTE J1908+094 & $0.75 \pm 0.09$ & & {$[350]$} \\
Swift J1910.2-0546 & $\leq-0.32$ & & {$[352]$} \\
GRS 1915+105 & $0.98 \pm 0.01$ & $\geq 0.95$ & {$[238,275]$} \\
Cygnus X-1 & $0.97_{-0.02}^{+0.01}$ & $\geq 0.95$ & $296]$ \\
\hline
\end{tabular}

Table 1: The errors in the table above generally reflect $1 \sigma$ statistical errors. Systematic errors are likely to be larger, and due to a combination of modeling uncertainties (e.g. the ability of the data to constrain the emissivity for reflection-based measurements, or the degree and energy at which an underlying hard component turns-over at low energy when modeling the disk continuum) and theoretical uncertainties (the degree to which a real fluid disk respects the test particle ISCO). The reflection-based spin measurement quoted for XTE J1550-564 is a composite value based on two recent complementary works; other values in this table are based on single measurements from the best available current research.

[363] reach their full sensitivities. Depending on the optimism of the modelers, predicted rates of detected compact object coalescence range from a few tenths per year to a few hundred per year [364]. These events, which include the analogs of the known double neutron star binaries as well as currently unknown but likely neutron star - black hole binaries and double black hole binaries, will open a new window onto compact objects. For our purposes, such detections will provide neutron star and black hole mass and spin estimates that will at a minimum have different systematics than existing determinations and at best will provide cleaner measurements than we have today. In this section we discuss how masses and spins can be obtained from gravitational waveforms, and the implications of the expected 


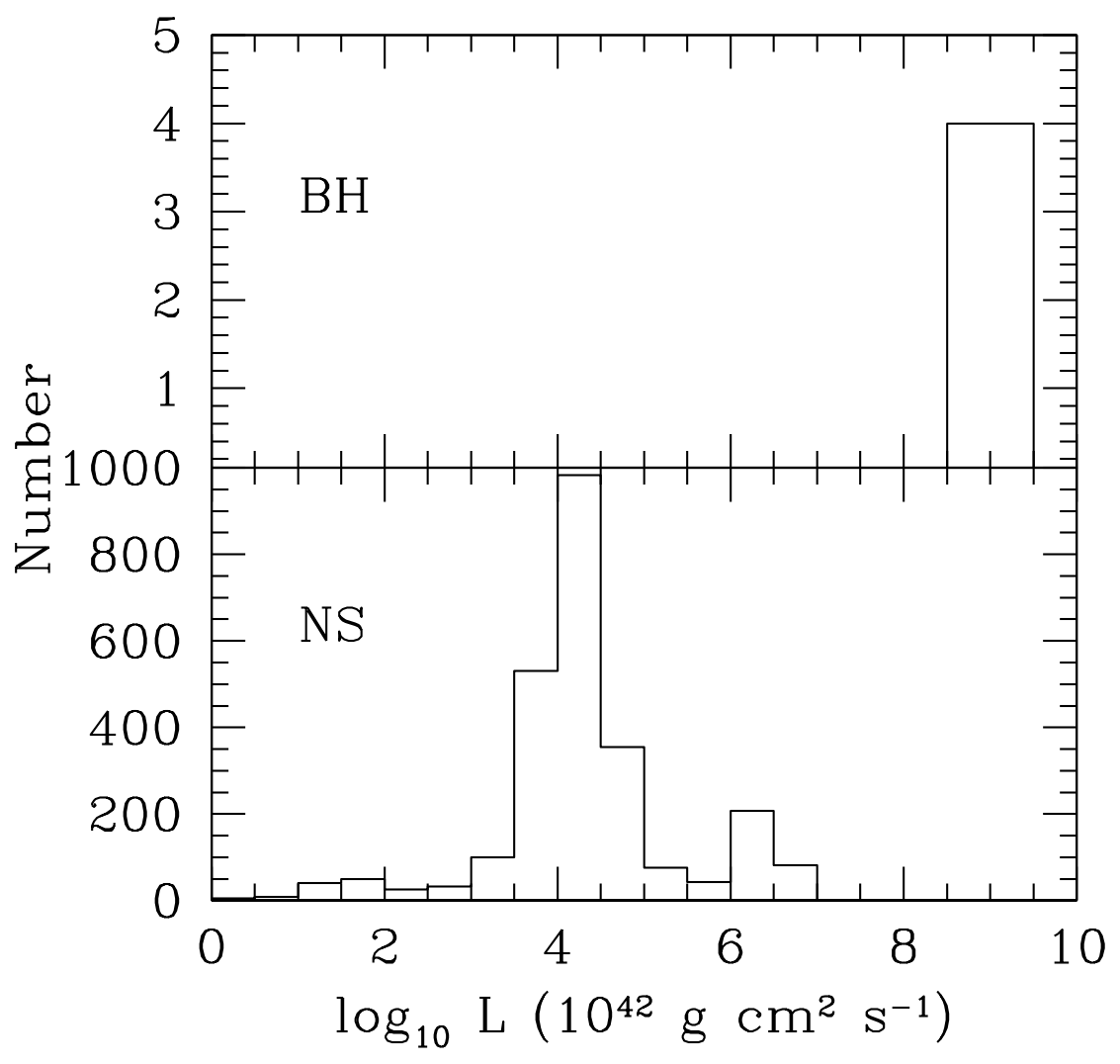

Figure 5: Comparison of the spin angular momenta of neutron stars and black holes. The key point is that even the fastest-spinning neutron stars have angular momenta two orders of magnitude less than the currently estimated angular momenta of black holes. This implies that there is a significant difference in the formation process; fallback from the pre-supernova star for black holes is one candidate. Here we assume a moment of inertia of $1.5 \times 10^{45} \mathrm{~g} \mathrm{~cm}^{2} \mathrm{~s}^{-1}$ for all the neutron stars (reasonable for a $\sim 1.5 M_{\odot}$ star; see [353]). For the black holes, we take the spin parameters for the black holes listed in Table 1 (averaging the disk reflection and disk continuum estimates if both are available) that also have mass estimates. This leaves us with eight sources: M33 X-7 (mass from 354]), LMC X-1 355], A0620-00 [356], 4U 1543-475 357], XTE J1550-654 358], GRO J1644-40 106], GRS 1915+105 [359], and Cyg X-1 299].

greatly expanded sample of well-measured compact objects. We will begin 
by considering nonspinning systems, then consider the effects of spin.

\subsection{Mass measurements from gravitational waves}

Gravitational radiation is analogous to electromagnetic radiation except that the equivalents of electric dipole and magnetic dipole radiation vanish. This is because the mass-energy version of electric dipole radiation is eliminated due to linear momentum conservation, and the equivalent of magnetic dipole radiation vanishes due to angular momentum conservation. Thus the lowest order radiation comes from variation of the mass-energy quadrupole. Binary systems naturally have time-variable quadrupoles, so they are potentially strong sources of gravitational radiation.

A classic study of gravitational waves from point-mass binaries was completed by Peters [365] to lowest order in the motion. Effectively, he assumed the adiabatic limit, in which the radiation takes away only a small fraction of the orbital angular momentum and energy in an orbit, so he was able to integrate the energy and angular momentum losses in one orbit of a Kepler ellipse. He found that the semimajor axis and eccentricity evolve as

$$
\begin{aligned}
\frac{d a}{d t} & =-\frac{64}{5} \frac{G^{3} m_{1} m_{2}\left(m_{1}+m_{2}\right)}{c^{5} a^{3}\left(1-e^{2}\right)^{7 / 2}}\left(1+73 e^{2} / 24+37 e^{4} / 96\right) \\
\frac{d e}{d t} & =-\frac{304}{15} e \frac{G^{3} m_{1} m_{2}\left(m_{1}+m_{2}\right)}{c^{5} a^{4}\left(1-e^{2}\right)^{5 / 2}}\left(1+121 e^{2} / 304\right) .
\end{aligned}
$$

Here $m_{1}$ and $m_{2}$ are the masses of the two objects. Thus orbits circularize as they shrink. This can be understood in the impulse approximation: the rate of gravitational radiation is a strong positive function of the relative speed of the objects, which is maximized at the pericenter. In the limit that energy and angular momentum are only lost at the pericenter, the impulse approximation tells us that the pericenter distance is conserved while the apocenter distance shrinks, so the eccentricity decreases.

When we observe the waves from such a system we see frequencies, so it is convenient to use $\omega=\left(G M / a^{3}\right)^{1 / 2}$, where $M=m_{1}+m_{2}$ is the total mass of the system. It is also standard to use the symmetric mass ratio $\eta \equiv m_{1} m_{2} / M^{2}$. Then we find

$$
\begin{gathered}
\frac{d \omega}{d t}=\frac{96}{5} \omega^{11 / 3} \frac{\eta(G M)^{5 / 3}}{c^{5}\left(1-e^{2}\right)^{7 / 2}}\left(1+73 e^{2} / 24+37 e^{4} / 96\right) \\
\frac{d e}{d t}=\frac{-304}{15} e \omega^{8 / 3} \frac{\eta(G M)^{5 / 3}}{c^{5}\left(1-e^{2}\right)^{5 / 2}}\left(1+121 e^{2} / 304\right) .
\end{gathered}
$$

We therefore see that the masses only appear in the combination $\eta(G M)^{5 / 3}$, or $\left(G M_{\mathrm{ch}}\right)^{5 / 3}$, where the "chirp mass" is $M_{\mathrm{ch}} \equiv \eta^{3 / 5} M$. The chirp mass, 
named thus because it dictates the rate of frequency sweep, can be determined with superior accuracy $(<1 \%$; e.g., [366]) by essentially counting cycles. It will be the most accurately determined parameter for binaries detected with gravitational radiation.

It is much more difficult to determine both masses separately, because different mass combinations only appear in higher-order, and thus much weaker, terms in the post-Newtonian expansion of the motion. The challenge will be particularly great for double neutron star binaries, or any binary where the components have comparable masses. The reason is that $\eta$ has a maximum (of 0.25) for equal-mass binaries, and thus has very little variation over a broad range of comparable masses; for example, $\eta=0.24$ for a mass ratio of $1.5: 1$. Thus over almost the entire range of plausible double neutron star mass ratios, $\eta$ is within a few percent of its maximum value. Given that $\eta$ can be measured at all only due to weak higher-order effects, extremely high signal to noise ratios will be required to disentangle the component masses in a double neutron star binary.

On a somewhat more optimistic note, if one can be sure that a given system has two neutron stars instead of a neutron star and a black hole or two low-mass black holes, then measurement of the chirp mass provides a highprecision measurement of the total mass, and consequently a strong lower limit on the more massive object of the pair. This is because $M=\eta^{-3 / 5} M_{\mathrm{ch}}$, so if $M_{\mathrm{ch}}$ is known then the additional uncertainty on $M$ is just $2-3 \%$ larger for plausible neutron star mass ratios. $M / 2$ is then clearly a lower limit on the mass of the more massive neutron star. Thus if a confirmed double neutron star system has a large enough chirp mass, it could raise the known mass upper limit for neutron stars.

The difficulty, of course, is in being sure that the system has two neutron stars. If a coincident gamma-ray burst is seen then at least one of the objects must have been a neutron star. Such a burst would argue against either object being a black hole with mass $>10 M_{\odot}$, because the neutron star would just be swallowed [367], but recent fully general relativistic simulations of the inspiral of neutron stars into low-mass black holes, particularly when the hole spins rapidly and the orbit is prograde with respect to the hole, suggest that bursts or other electromagnetic signals might be possible 368, 369, 370, 371, 372, 373, 374, 375, 376, 377, 378, 379, 380, 381, 382, 383, 384, 385, 386, 387, 388, 389, 390]. More research is required.

Black hole - neutron star binaries have component masses that differ more than in double neutron star binaries, so a given amount of uncertainty 
in $\eta$ does not translate to such enormous uncertainties in the individual masses. However, the rate of such coalescences is highly uncertain because we do not know of any black hole - neutron star examples in our galaxy, and in addition spin-orbit coupling and precession may greatly complicate the analysis of these systems. We now discuss these effects.

\subsection{The effects of spin}

As we mentioned in Section 1, the spin frequencies of known neutron stars extend up to $716 \mathrm{~Hz}$ [47], which could correspond to a dimensionless spin $\hat{a} \sim 0.2-0.3$ depending on the star's mass and radius. However, the highest spin frequency of a neutron star in a binary with another neutron star is only $\sim 44 \mathrm{~Hz}$. This is understandable in terms of the evolution of the system: our current understanding of the fastest-rotating neutron stars suggests that their rapid rotation is produced after their birth in supernovae, via longterm accretion from a companion that also somehow reduces their magnetic moments by orders of magnitude (see [8] for a review). A companion to a neutron star that evolves into another neutron star has a short enough lifetime that it cannot transfer much mass [67], and thus such spinup will be difficult. If the neutron star is paired with a black hole that evolved before the neutron star, then clearly there will be no spinup after the formation of the neutron star, meaning that the neutron stars in NS-BH binaries are likely to have $\hat{a} \ll 1$. Such spins could affect the long-term phase evolution of the system, because spin-orbit coupling enters at the relatively low $1.5 \mathrm{PN}$ order [391], but these spins will produce only minor effects at high frequencies. Thus mass and radius inferences for double neutron star systems are unlikely to be affected much by spins.

In contrast, as we described in Section $[3$, current estimates of the spins of stellar-mass black holes suggest that for them $\hat{a} \sim 1$ is possible. There are no known BH-NS or BH-BH binaries, so extrapolation from BH LMXBs and HMXBs is necessary, but if black hole spins in compact binaries are similarly high then they could have a considerable impact on the gravitational radiation waveforms and the precision with which the properties of the system can be inferred. This is especially true if black hole spins in compact binaries can be significantly misaligned with the orbital axis (as we discuss in Section 3 , some observations suggest that such misalignment might exist for LMXBs).

To see this, consider a neutron star of mass $1.5 M_{\odot}$ spiraling into a $10 M_{\odot}$ black hole that has $\hat{a}=0.9$. For such a system, the spin angular momentum

of the black hole exceeds the orbital angular momentum of the neutron star if 
the orbital radius of the NS is less than $\sim 600 \mathrm{~km}$, or $\sim 35 M$ in gravitational units. Thus in an extreme case the neutron star orbit could be flipped by the spin of the black hole when the star is close enough. The effect on the waveform of a comparable-mass BH-BH binary will be more modest, but misaligned spins could still induce considerable precession (see [392, 393, 394] for references for this and the following discussion).

Such precession can either help or hurt parameter determinations, depending on the details. For example, compared to a spinless and thus nonprecessing binary, for which the orbital plane is fixed, degeneracies between, e.g., the sky direction and the normal to the orbit can be broken when the binary precesses. This could help in the localization of an electromagnetic counterpart. However, rapid spins can also change the overall phase evolution in ways that can be degenerate with a different chirp mass than the actual one. If, contrary to expectations, there are rapidly-spinning neutron stars in compact binaries, their oblateness could complicate analysis of the high-frequency portion of the coalescence. For the expected essentially nonspinning neutron stars in such systems, the recently elucidated relations between the moment of inertia, Love number, and quadrupole moment (the so-called I-Love-Q relations; see [353, 395, 396]) will help in the inference of neutron star masses and radii.

The phase space produced when spins are included is large enough that it has not yet been possible to do a comprehensive study. Some preliminary results [392, 393, 394] suggest that the precision with which masses can be inferred will not be problematically reduced in systems with spins. Thus the challenge might not be parameter estimation once a detection is made, but rather the detection itself, given the expanded library of waveforms that will be needed to accommodate spin.

\section{Conclusions}

We have reviewed current estimates of the masses and spins of neutron stars and black holes, in isolation, and in binary systems with a variety of possible companions. Taking a broad view of recent results and progress, it is increasingly clear that stellar-mass compact objects are vital laboratories for the exploration of strong gravity and nuclear physics.

Precise neutron star masses, which until recently were limited to the narrow $1.25 M_{\odot}-1.44 M_{\odot}$ range thus far observed from double neutron star 
systems, have now been extended to $2 M_{\odot}[112,44]$. This has immediate implications for the equation of state of cold supranuclear matter; in particular, it requires that this matter be relatively hard, whatever its precise composition. Truly strong constraints on this matter await reliable neutron star radius estimates [16], but the increasing precision of millisecond pulsar timing suggests that measurements of Shapiro delay and other post-Keplerian parameters will in the near future be possible for a broader range of neutron star systems. If any of those systems have well-determined masses that are significantly higher than the current $2 M_{\odot}$ maximum then equations of state involving exotic non-nucleonic components will be strongly challenged [397].

The masses of stellar black holes are not as easy to measure with precision, because they require a special set of circumstances (a transient system that fully shuts off its accretion to allow measurements of ellipsoidal light variations). Sensitivity is not the limiting factor (at least in the Galaxy), nor is timing. Rather, patience is the key: more sources in the Galaxy means waiting a greater time for currently inactive transients to reveal themselves. Qualitative advances with current data may be possible if high-frequency QPOs are understood from first-principles simulations, so in this field we may primarily be waiting on theoretical developments.

An intriguing question is whether there is actually a mass gap between neutron stars and black holes. Some initial analyses imply that such a gap exists (or at least that low-mass black holes are much less common than might be suggested by the initial mass function of main sequence stars), but more recent work has suggested that at least part of this result could be due to selection effects. Whether or not there is a gap, an understanding of the true black hole and neutron star mass distributions at formation will give us key information about the evolution of massive stars and the nature of core collapse.

The current spin of any pulsar is by definition easy to measure. It is much more challenging to determine the natal spin of such neutron stars. Estimates have been made in a few cases based on, for example, the argument that an extremely rapid initial spin would produce a highly luminous nebula. Such arguments suggest in a handful of cases that the initial spin periods are more like tenths of a second than milliseconds, but given that this is based on $\sim 10$ out of $\sim 2000$ pulsars we must be wary of selection effects.

Unlike neutron star spins (and masses), black hole spins are believed to essentially be natal, with one possible exception being that the spins of black holes in a hyperaccreting Thorne-Żytkow phase might be altered 
significantly. For the majority of black holes, therefore, if the current spin can be measured it serves as a record of the formation process. Estimates of black hole spin are necessarily far more indirect than they are for neutron stars. We have reviewed two different methods, one involving characterization of Fe $\mathrm{K} \alpha$ lines and the other using continuum spectral fits. Both methods have strengths and weaknesses, but there is reason for cautious optimism because spin estimates for the overlap sources are in fairly good agreement. Other methods, e.g., those involving QPO modeling or polarization, will require significant theoretical or instrumental development to be reliable, but when this happens it will produce an independent channel of information. In any case, current estimates of black hole angular momenta indicate that most are far beyond any possible angular momentum for neutron stars, which has implications for the rotation distribution of their progenitor stars.

Measurements of black hole spin also provide an avenue by which relativistic jet launching mechanisms can be explored. The Blandford-Znajek effect is a particularly appealing jet-launching mechanism that taps the spin of the black hole [270]; if it is correct, then proxies for jet power should be positively correlated with spin values. Based on very few sources, correlations between proxies for jet power and black hole spin have been claimed ([398]; however, other work suggests that the apparent relation is an artifact of selection effects $([399])$. Deeper appreciation of the mechanism may not even predict a simple relationship between spin and a jet proxy like radio flux. Spin might set the maximum possible power that a jet can carry; however, instantaneous jet power is likely determined by how the disk and ergosphere couple, and this must be a function of several accretion parameters that affect the magnetic field. That is, spin may set the maximum horsepower of the engine, but the accretion flow is a throttle (see, e.g., [260]). A large collection of jet proxy measurements for a collection of sources with known spins might be expected to fill a large portion of a $P_{\text {jet }}$ versus $a$ plane, with evidence of spin driving jets manifesting as an upper envelope.

An exciting near-future prospect is the entry of gravitational wave observations into the mass and spin constraint game. For the stellar-mass objects we consider, this will be the domain of ground-based detectors rather than the equally exciting developments in, e.g., pulsar timing arrays. The initial detections of binary compact object coalescence will measure precisely only the chirp masses of the binaries, rather than the individual masses. However, as sensitivity (particularly at high frequencies) improves, detection of higher-order effects will allow the measurements of individual masses. 
This will clarify whether, for example, the current tight range of neutron star masses in double neutron star systems is maintained (perhaps due to a narrow evolutionary channel for such systems) or whether occasional much higher-mass neutron stars will be seen. Spin measurements of neutron stars will be challenging if they rotate as slowly as the stars in currently known double neutron star systems. There is reason to be optimistic that black hole spins will be easier to measure, and it will be especially interesting to determine the distribution of relative spin orientations of binary black holes. This is because different origins (evolution from a massive binary, versus dynamical capture in a dense stellar environment) imply very different distributions of the relative orientations.

Finally, it is important to emphasize that electromagnetic and gravitational wave measurements of neutron stars and black holes will provide strongly complementary constraints on the nature of strong gravity. The detection of gravitational radiation, particularly of a double black hole coalescence, will yield direct constraints on the nature of strong gravity, and in particular whether general relativity can pass such clean tests as it has passed the weak gravity tests available from binary pulsars and in our Solar System. However, such observations cannot by themselves test our theories of how matter and electromagnetic fields interact in strong gravity. This information can only come from current and future electromagnetic observations of compact objects, especially those that are accreting. For example, the excellent fits to current iron line and continuum observations both rely on the existence of an innermost stable circular orbit, which is a key prediction of models of strong gravity. Another example is that if we can detect a pulsar in a binary around a black hole, this will produce new levels of precision in black hole mass and possibly spin measurements as well as yielding new types of tests of the predictions of general relativity in weak gravity.

In general, the theoretical framework for and observations of compact object masses and spins have evolved rapidly. The extreme nature of the gravity and material properties of these systems mean that they are crucial testbeds for some of our most fundamental physical theories. We hope for and expect continued qualitative improvements in our understanding, and we look forward to the unanticipated in our study of neutron stars and black holes. 


\section{Acknowledgements}

We thank Charles Bailyn, Didier Barret, Ed Brown, Ed Cackett, Shane Davis, Jason Dexter, Zach Etienne, Julian Krolik, Alessandro Patruno, Chris Reynolds, and Jeremy Schnittman for helpful discussions and comments on an early version of this manuscript. We give special thanks to our editor, Marc Kamionkowski, for proposing this review and for many helpful suggestions. MCM was supported in part by NASA ATP grant NNX12AG29G. This work was also supported in part by National Science Foundation Grant No. PHYS-1066293 and the hospitality of the Aspen Center for Physics. 


\section{References}

[1] A. Hewish, S. J. Bell, J. D. H. Pilkington, P. F. Scott, R. A. Collins, Observation of a Rapidly Pulsating Radio Source, Nature 217 (1968) 709-713.

[2] E. E. Salpeter, Accretion of Interstellar Matter by Massive Objects., ApJ 140 (1964) 796-800.

[3] D. Lynden-Bell, Galactic Nuclei as Collapsed Old Quasars, Nature 223 (1969) 690-694.

[4] S. Bowyer, E. T. Byram, T. A. Chubb, H. Friedman, Cosmic X-ray Sources, Science 147 (1965) 394-398.

[5] B. L. Webster, P. Murdin, Cygnus X-1-a Spectroscopic Binary with a Heavy Companion?, Nature 235 (1972) 37-38.

[6] C. T. Bolton, Identification of Cygnus X-1 with HDE 226868, Nature 235 (1972) 271-273.

[7] H. Tananbaum, H. Gursky, E. Kellogg, R. Giacconi, C. Jones, Observation of a Correlated X-Ray Transition in Cygnus X-1, ApJL 177 (1972) L5.

[8] D. R. Lorimer, Binary and Millisecond Pulsars, Living Reviews in Relativity 11 (2008) 8.

[9] L. Kreidberg, C. D. Bailyn, W. M. Farr, V. Kalogera, Mass Measurements of Black Holes in X-Ray Transients: Is There a Mass Gap?, ApJ 757 (2012) 36.

[10] C. S. Reynolds, M. A. Nowak, Fluorescent iron lines as a probe of astrophysical black hole systems, Phys. Rep. 377 (2003) 389-466.

[11] J. E. McClintock, R. Narayan, S. W. Davis, L. Gou, A. Kulkarni, J. A. Orosz, R. F. Penna, R. A. Remillard, J. F. Steiner, Measuring the spins of accreting black holes, Classical and Quantum Gravity 28 (2011) 114009. 
[12] L. Blanchet, Gravitational Radiation from Post-Newtonian Sources and Inspiralling Compact Binaries, Living Reviews in Relativity 17 (2014) 2 .

[13] B. S. Sathyaprakash, B. F. Schutz, Physics, Astrophysics and Cosmology with Gravitational Waves, Living Reviews in Relativity 12 (2009) 2 .

[14] D. Psaltis, Probes and Tests of Strong-Field Gravity with Observations in the Electromagnetic Spectrum, Living Reviews in Relativity 11 (2008) 9 .

[15] J. M. Lattimer, Neutron stars and the dense matter equation of state, Ap\&SS 336 (2011) 67-74.

[16] M. C. Miller, Astrophysical Constraints on Dense Matter in Neutron Stars, arXiv:1312.0029 (2013).

[17] B. R. McNamara, P. E. J. Nulsen, M. W. Wise, D. A. Rafferty, C. Carilli, C. L. Sarazin, E. L. Blanton, The heating of gas in a galaxy cluster by X-ray cavities and large-scale shock fronts, Nature 433 (2005) 45-47.

[18] S. R. Kulkarni, P. Hut, S. McMillan, Stellar black holes in globular clusters, Nature 364 (1993) 421-423.

[19] S. Sigurdsson, L. Hernquist, Primordial black holes in globular clusters, Nature 364 (1993) 423-425.

[20] S. F. Portegies Zwart, S. L. W. McMillan, Black Hole Mergers in the Universe, ApJL 528 (2000) L17-L20.

[21] R. M. O'Leary, F. A. Rasio, J. M. Fregeau, N. Ivanova, R. O'Shaughnessy, Binary Mergers and Growth of Black Holes in Dense Star Clusters, ApJ 637 (2006) 937-951.

[22] S. Banerjee, H. Baumgardt, P. Kroupa, Stellar-mass black holes in star clusters: implications for gravitational wave radiation, MNRAS 402 (2010) 371-380.

[23] T. J. Maccarone, A. Kundu, S. E. Zepf, K. L. Rhode, A black hole in a globular cluster, Nature 445 (2007) 183-185. 
[24] N. J. Brassington, G. Fabbiano, S. Blake, A. Zezas, L. Angelini, R. L. Davies, J. Gallagher, V. Kalogera, D.-W. Kim, A. R. King, A. Kundu, G. Trinchieri, S. Zepf, The X-ray Spectra of the Luminous LMXBs in NGC 3379: Field and Globular Cluster Sources, ApJ 725 (2010) $1805-1823$.

[25] I. C. Shih, A. Kundu, T. J. Maccarone, S. E. Zepf, T. D. Joseph, A Variable Black Hole X-ray Source in an NGC 1399 Globular Cluster, ApJ 721 (2010) 323-328.

[26] R. Barnard, M. Garcia, Z. Li, F. Primini, S. S. Murray, Four New Black Hole Candidates Identified in M31 Globular Clusters with Chandra and XMM-Newton, ApJ 734 (2011) 79.

[27] T. J. Maccarone, A. Kundu, S. E. Zepf, K. L. Rhode, A new globular cluster black hole in NGC 4472, MNRAS 410 (2011) 1655-1659.

[28] J. Strader, L. Chomiuk, T. J. Maccarone, J. C. A. Miller-Jones, A. C. Seth, Two stellar-mass black holes in the globular cluster M22, Nature 490 (2012) 71-73.

[29] M. Morscher, S. Umbreit, W. M. Farr, F. A. Rasio, Retention of Stellar-mass Black Holes in Globular Clusters, ApJL 763 (2013) L15.

[30] P. G. Breen, D. C. Heggie, Dynamical evolution of black hole subsystems in idealized star clusters, MNRAS 432 (2013) 2779-2797.

[31] M. Trenti, R. van der Marel, No energy equipartition in globular clusters, MNRAS 435 (2013) 3272-3282.

[32] P. G. Breen, D. C. Heggie, On black hole subsystems in idealized nuclear star clusters, MNRAS 436 (2013) 584-589.

[33] S. A. Colgate, A. G. Petschek, J. T. Kriese, The light curve of type I Supernovae, in: R. Meyerott, H. G. Gillespie (Eds.), Supernovae Spectra, volume 63 of American Institute of Physics Conference Series, pp. $7-14$.

[34] B. J. Carr, S. W. Hawking, Black holes in the early Universe, MNRAS 168 (1974) 399-416. 
[35] B. J. Carr, The primordial black hole mass spectrum, ApJ 201 (1975) $1-19$.

[36] D. K. Nadezhin, I. D. Novikov, A. G. Polnarev, The hydrodynamics of primordial black hole formation, Sov. Astr. 22 (1978) 129-138.

[37] G. V. Bicknell, R. N. Henriksen, Self-similar growth of primordial black holes. II - General sound speed, ApJ 225 (1978) 237-251.

[38] I. D. Novikov, A. G. Polnarev, A. A. Starobinskii, I. B. Zeldovich, Primordial black holes, A\&A 80 (1979) 104-109.

[39] H. Kodama, M. Sasaki, K. Sato, Abundance of Primordial Holes Produced by Cosmological First-Order Phase Transition, Progress of Theoretical Physics 68 (1982) 1979-1998.

[40] R. V. Konoplich, S. G. Rubin, A. S. Sakharov, M. Y. Khlopov, Formation of black holes in first-order phase transitions in the Universe, Astronomy Letters 24 (1998) 413-417.

[41] K. Jedamzik, J. C. Niemeyer, Primordial black hole formation during first-order phase transitions, Phys. Rev. D 59 (1999) 124014.

[42] A. M. Green, A. R. Liddle, Critical collapse and the primordial black hole initial mass function, Phys. Rev. D 60 (1999) 063509.

[43] A. G. Lyne, M. Burgay, M. Kramer, A. Possenti, R. N. Manchester, F. Camilo, M. A. McLaughlin, D. R. Lorimer, N. D'Amico, B. C. Joshi, J. Reynolds, P. C. C. Freire, A Double-Pulsar System: A Rare Laboratory for Relativistic Gravity and Plasma Physics, Science 303 (2004) $1153-1157$.

[44] J. Antoniadis, P. C. C. Freire, N. Wex, T. M. Tauris, R. S. Lynch, M. H. van Kerkwijk, M. Kramer, C. Bassa, V. S. Dhillon, T. Driebe, J. W. Hessels, V. M. Kaspi, V. I. Kondratiev, N. Langer, T. R. Marsh, M. A. McLaughlin, T. T. Pennucci, S. M. Ransom, I. H. Stairs, J. van Leeuwen, J. P. W. Verbiest, D. G. Whelan, A massive pulsar in a compact relativistic binary., Science 340 (2013) 448.

[45] K. Hebeler, J. M. Lattimer, C. J. Pethick, A. Schwenk, Equation of State and Neutron Star Properties Constrained by Nuclear Physics and Observation, ApJ 773 (2013) 11. 
[46] C. O. Heinke, H. N. Cohn, P. M. Lugger, N. A. Webb, W. C. G. Ho, J. Anderson, S. Campana, S. Bogdanov, D. Haggard, A. M. Cool, J. E. Grindlay, Improved Mass and Radius Constraints for Quiescent Neutron Stars in Omega Cen and NGC 6397, ArXiv e-prints (2014).

[47] J. W. T. Hessels, S. M. Ransom, I. H. Stairs, P. C. C. Freire, V. M. Kaspi, F. Camilo, A Radio Pulsar Spinning at $716 \mathrm{~Hz}$, Science 311 (2006) 1901-1904.

[48] G. B. Cook, S. L. Shapiro, S. A. Teukolsky, Rapidly rotating neutron stars in general relativity: Realistic equations of state, ApJ 424 (1994) 823-845.

[49] M. C. Miller, E. J. M. Colbert, Intermediate-Mass Black Holes, International Journal of Modern Physics D 13 (2004) 1-64.

[50] K. S. Thorne, Disk-Accretion onto a Black Hole. II. Evolution of the Hole, ApJ 191 (1974) 507-520.

[51] C. F. Gammie, S. L. Shapiro, J. C. McKinney, Black Hole Spin Evolution, ApJ 602 (2004) 312-319.

[52] J. H. Krolik, J. F. Hawley, S. Hirose, Magnetically Driven Accretion Flows in the Kerr Metric. IV. Dynamical Properties of the Inner Disk, ApJ 622 (2005) 1008-1023.

[53] J. M. Bardeen, W. H. Press, S. A. Teukolsky, Rotating Black Holes: Locally Nonrotating Frames, Energy Extraction, and Scalar Synchrotron Radiation, ApJ 178 (1972) 347-370.

[54] A. Burrows, Colloquium: Perspectives on core-collapse supernova theory, Reviews of Modern Physics 85 (2013) 245-261.

[55] F. P. Pijpers, Helioseismic determination of the solar gravitational quadrupole moment, MNRAS 297 (1998) L76-L80.

[56] H. Spruit, E. S. Phinney, Birth kicks as the origin of pulsar rotation, Nature 393 (1998) 139-141.

[57] C. Thompson, R. C. Duncan, Neutron star dynamos and the origins of pulsar magnetism, ApJ 408 (1993) 194-217. 
[58] D. A. Neufeld, P. R. Maloney, S. Conger, Water maser emission from X-ray-heated circumnuclear gas in active galaxies, ApJL 436 (1994) L127.

[59] O. Blaes, O. Warren, P. Madau, Accreting, Isolated Neutron Stars. III. Preheating of Infalling Gas and Cometary H II Regions, ApJ 454 (1995) 370.

[60] K. S. Thorne, A. N. Zytkow, Red giants and supergiants with degenerate neutron cores, ApJL 199 (1975) L19-L24.

[61] K. S. Thorne, A. N. Zytkow, Stars with degenerate neutron cores. I Structure of equilibrium models, ApJ 212 (1977) 832-858.

[62] E. M. Levesque, P. Massey, A. N. Żytkow, N. Morrell, Discovery of a Thorne-Żytkow object candidate in the Small Magellanic Cloud, MNRAS 443 (2014) L94-L98.

[63] S. Ichimaru, Bimodal behavior of accretion disks - Theory and application to Cygnus X-1 transitions, ApJ 214 (1977) 840-855.

[64] M. C. Begelman, D. L. Meier, Thick accretion disks - Self-similar, supercritical models, ApJ 253 (1982) 873-896.

[65] R. A. Chevalier, Neutron star accretion in a supernova, ApJ 346 (1989) $847-859$.

[66] G. E. Brown, J. C. Weingartner, Accretion onto and radiation from the compact object formed in SN 1987A, ApJ 436 (1994) 843-847.

[67] I. Iben, Jr., A. V. Tutukov, L. R. Yungelson, A Model of the Galactic X-Ray Binary Population. I. High-Mass X-Ray Binaries, ApJS 100 (1995) 217.

[68] S. L. Shapiro, A. P. Lightman, Black holes in X-ray binaries - Marginal existence and rotation reversals of accretion disks, ApJ 204 (1976) 555560.

[69] A. R. King, U. Kolb, The evolution of black hole mass and angular momentum, MNRAS 305 (1999) 654-660. 
[70] A. R. King, S. H. Lubow, G. I. Ogilvie, J. E. Pringle, Aligning spinning black holes and accretion discs, MNRAS 363 (2005) 49-56.

[71] J. M. Bardeen, J. A. Petterson, The Lense-Thirring Effect and Accretion Disks around Kerr Black Holes, ApJL 195 (1975) L65.

[72] J. C. B. Papaloizou, J. E. Pringle, The time-dependence of non-planar accretion discs, MNRAS 202 (1983) 1181-1194.

[73] J. E. Pringle, A simple approach to the evolution of twisted accretion discs, MNRAS 258 (1992) 811-818.

[74] P. Natarajan, J. E. Pringle, The Alignment of Disk and Black Hole Spins in Active Galactic Nuclei, ApJL 506 (1998) L97-L100.

[75] P. J. Armitage, P. Natarajan, Lense-Thirring Precession of Accretion Disks around Compact Objects, ApJ 525 (1999) 909-914.

[76] G. I. Ogilvie, The non-linear fluid dynamics of a warped accretion disc, MNRAS 304 (1999) 557-578.

[77] R. P. Nelson, J. C. B. Papaloizou, Hydrodynamic simulations of the Bardeen-Petterson effect, MNRAS 315 (2000) 570-586.

[78] G. Lodato, J. E. Pringle, Warp diffusion in accretion discs: a numerical investigation, MNRAS 381 (2007) 1287-1300.

[79] G. Lodato, D. J. Price, On the diffusive propagation of warps in thin accretion discs, MNRAS 405 (2010) 1212-1226.

[80] C. Nixon, A. King, D. Price, J. Frank, Tearing up the Disk: How Black Holes Accrete, ApJL 757 (2012) L24.

[81] K. A. Sorathia, J. H. Krolik, J. F. Hawley, Relaxation of Warped Disks: The Case of Pure Hydrodynamics, ApJ 768 (2013) 133.

[82] K. A. Sorathia, J. H. Krolik, J. F. Hawley, Magnetohydrodynamic Simulation of a Disk Subjected to Lense-Thirring Precession, ApJ 777 (2013) 21.

[83] P. C. Fragile, O. M. Blaes, P. Anninos, J. D. Salmonson, Global General Relativistic Magnetohydrodynamic Simulation of a Tilted Black Hole Accretion Disk, ApJ 668 (2007) 417-429. 
[84] J. Michell, a Inquiry into the Probable Parallax, and Magnitude of the Fixed Stars, from the Quantity of Light Which They Afford us, and the Particular Circumstances of Their Situation, by the Rev. John Michell, B. D. F. R. S., Royal Society of London Philosophical Transactions Series I 57 (1767) 234-264.

[85] T. M. Brown, R. L. Gilliland, Asteroseismology, Ann. Rev. Astron. Astrophys. 32 (1994) 37-82.

[86] D. E. Winget, S. O. Kepler, Pulsating White Dwarf Stars and Precision Asteroseismology, Ann. Rev. Astron. Astrophys. 46 (2008) 157-199.

[87] S. Mathur, T. S. Metcalfe, M. Woitaszek, H. Bruntt, G. A. Verner, J. Christensen-Dalsgaard, O. L. Creevey, G. Doğan, S. Basu, C. Karoff, D. Stello, T. Appourchaux, T. L. Campante, W. J. Chaplin, R. A. García, T. R. Bedding, O. Benomar, A. Bonanno, S. Deheuvels, Y. Elsworth, P. Gaulme, J. A. Guzik, R. Handberg, S. Hekker, W. Herzberg, M. J. P. F. G. Monteiro, L. Piau, P.-O. Quirion, C. Régulo, M. Roth, D. Salabert, A. Serenelli, M. J. Thompson, R. Trampedach, T. R. White, J. Ballot, I. M. Brandão, J. MolendaŻakowicz, H. Kjeldsen, J. D. Twicken, K. Uddin, B. Wohler, A Uniform Asteroseismic Analysis of 22 Solar-type Stars Observed by Kepler, ApJ 749 (2012) 152.

[88] A. Miglio, C. Chiappini, T. Morel, M. Barbieri, W. J. Chaplin, L. Girardi, J. Montalbán, M. Valentini, B. Mosser, F. Baudin, L. Casagrande, L. Fossati, V. S. Aguirre, A. Baglin, Galactic archaeology: mapping and dating stellar populations with asteroseismology of red-giant stars, MNRAS 429 (2013) 423-428.

[89] S. Mao, Astrophysical applications of gravitational microlensing, Research in Astronomy and Astrophysics 12 (2012) 947-972.

[90] L. D. Landau, On the Theory of Stars, Phys. Z. Sowjet 1 (1932) 285.

[91] S. Chandrasekhar, The Maximum Mass of Ideal White Dwarfs, ApJ 74 (1931) 81.

[92] J. R. Oppenheimer, G. M. Volkoff, On Massive Neutron Cores, Physical Review 55 (1939) 374-381. 
[93] I. M. Barbour, S. E. Morrison, E. G. Klepfish, J. B. Kogut, M.-P. Lombardo, The critical points of QCD at non-zero density, $\mathrm{T}=0, \mathrm{~g}=\infty$, Nuclear Physics B Proceedings Supplements 63 (1998) 439-441.

[94] M. Alford, New possibilities for QCD at finite density, Nuclear Physics B Proceedings Supplements 73 (1999) 161-166.

[95] S. Hands, Lattice matter, Nuclear Physics B Proceedings Supplements 106 (2002) 142-150.

[96] M. P. Lombardo, K. Splittorff, J. J. M. Verbaarschot, Lattice QCD and dense quark matter, arXiv:0912.4410 (2009).

[97] C. E. Rhoades, R. Ruffini, Maximum Mass of a Neutron Star, Physical Review Letters 32 (1974) 324-327.

[98] V. Kalogera, G. Baym, The Maximum Mass of a Neutron Star, ApJL 470 (1996) L61.

[99] C. J. Horowitz, E. F. Brown, Y. Kim, W. G. Lynch, R. Michaels, A. Ono, J. Piekarewicz, M. B. Tsang, H. H. Wolter, A way forward in the study of the symmetry energy: experiment, theory, and observation, ArXiv e-prints (2014).

[100] J. L. Friedman, J. R. Ipser, On the maximum mass of a uniformly rotating neutron star, ApJ 314 (1987) 594-597.

[101] D. J. Hegyi, The Damping of Differential Rotation in the Cores of Neutron Stars, in: M. D. Papagiannis (Ed.), Eighth Texas Symposium on Relativistic Astrophysics, volume 302 of Annals of the New York Academy of Sciences, p. 528.

[102] P. C. Joss, S. A. Rappaport, Neutron Stars in Interacting Binary Systems, Ann. Rev. Astron. Astrophys. 22 (1984) 537-592.

[103] R. Brucato, J. Kristian, Spectroscopic Observations of the Optical Candidate for Cygnus X-1, ApJL 179 (1973) L129.

[104] Y. Avni, Mass estimates from optical-light curves for binary X-ray sources, in: R. Giacconi, R. Ruffini (Eds.), Physics and Astrophysics of Neutron Stars and Black Holes, pp. 43-62. 
[105] J. E. McClintock, R. A. Remillard, The X-ray nova Centaurus X-4 Comparisons with A0620 - 00, ApJ 350 (1990) 386-394.

[106] J. Greene, C. D. Bailyn, J. A. Orosz, Optical and Infrared Photometry of the Microquasar GRO J1655-40 in Quiescence, ApJ 554 (2001) $1290-1297$.

[107] R. A. Hulse, J. H. Taylor, Discovery of a pulsar in a binary system, ApJL 195 (1975) L51-L53.

[108] P. C. C. Freire, Eccentric Binary Millisecond Pulsars, arXiv:0907.3219 (2009).

[109] J. H. Taylor, Jr., Millisecond pulsars - Nature's most stable clocks, IEEE Proceedings 79 (1991) 1054-1062.

[110] M. Kramer, I. H. Stairs, R. N. Manchester, M. A. McLaughlin, A. G. Lyne, R. D. Ferdman, M. Burgay, D. R. Lorimer, A. Possenti, N. D'Amico, J. M. Sarkissian, G. B. Hobbs, J. E. Reynolds, P. C. C. Freire, F. Camilo, Tests of General Relativity from Timing the Double Pulsar, Science 314 (2006) 97-102.

[111] E. S. Phinney, Pulsars as Probes of Newtonian Dynamical Systems, Royal Society of London Philosophical Transactions Series A 341 (1992) 39-75.

[112] P. B. Demorest, T. Pennucci, S. M. Ransom, M. S. E. Roberts, J. W. T. Hessels, A two-solar-mass neutron star measured using Shapiro delay, Nature 467 (2010) 1081-1083.

[113] G. E. Brown, H. A. Bethe, A Scenario for a Large Number of Low-Mass Black Holes in the Galaxy, ApJ 423 (1994) 659.

[114] C. M. Zhang, J. Wang, Y. H. Zhao, H. X. Yin, L. M. Song, D. P. Menezes, D. T. Wickramasinghe, L. Ferrario, P. Chardonnet, Study of measured pulsar masses and their possible conclusions, A\&A 527 (2011) A83.

[115] C. D. Bailyn, R. K. Jain, P. Coppi, J. A. Orosz, The Mass Distribution of Stellar Black Holes, ApJ 499 (1998) 367. 
[116] F. Özel, D. Psaltis, R. Narayan, J. E. McClintock, The Black Hole Mass Distribution in the Galaxy, ApJ 725 (2010) 1918-1927.

[117] W. M. Farr, N. Sravan, A. Cantrell, L. Kreidberg, C. D. Bailyn, I. Mandel, V. Kalogera, The Mass Distribution of Stellar-mass Black Holes, ApJ 741 (2011) 103.

[118] C. S. Kochanek, Failed Supernovae Explain the Compact Remnant Mass Function, ApJ 785 (2014) 28.

[119] R. N. Manchester, The Parkes Pulsar Timing Array, Chinese Journal of Astronomy and Astrophysics Supplement 6 (2006) 020000-147.

[120] A. A. Abdo, M. Ajello, A. Allafort, L. Baldini, J. Ballet, G. Barbiellini, M. G. Baring, D. Bastieri, A. Belfiore, R. Bellazzini, et al., The Second Fermi Large Area Telescope Catalog of Gamma-Ray Pulsars, ApJS 208 (2013) 17.

[121] A. Patruno, A. L. Watts, Accreting Millisecond X-Ray Pulsars, arXiv:1206.2727 (2012).

[122] D. K. Galloway, M. P. Muno, J. M. Hartman, D. Psaltis, D. Chakrabarty, Thermonuclear (Type I) X-Ray Bursts Observed by the Rossi X-Ray Timing Explorer, ApJS 179 (2008) 360-422.

[123] S. A. Olausen, V. M. Kaspi, The McGill Magnetar Catalog, ApJS 212 (2014) 6 .

[124] A. L. Watts, Thermonuclear Burst Oscillations, Ann. Rev. Astron. Astrophys. 50 (2012) 609-640.

[125] F. Pacini, Energy Emission from a Neutron Star, Nature 216 (1967) $567-568$.

[126] T. Gold, Rotating Neutron Stars as the Origin of the Pulsating Radio Sources, Nature 218 (1968) 731-732.

[127] A. K. Harding, The neutron star zoo, Frontiers of Physics 8 (2013) 679-692. 
[128] C. Thompson, R. C. Duncan, The soft gamma repeaters as very strongly magnetized neutron stars - I. Radiative mechanism for outbursts, MNRAS 275 (1995) 255-300.

[129] C. Thompson, R. C. Duncan, The Soft Gamma Repeaters as Very Strongly Magnetized Neutron Stars. II. Quiescent Neutrino, X-Ray, and Alfven Wave Emission, ApJ 473 (1996) 322.

[130] S. B. Popov, R. Turolla, Initial spin periods of neutron stars in supernova remnants, Ap\&SS 341 (2012) 457-464.

[131] R. N. Manchester, J. H. Taylor, Pulsars., 1977.

[132] W. C. G. Ho, N. Andersson, Rotational evolution of young pulsars due to superfluid decoupling, Nature Physics 8 (2012) 787-789.

[133] J. A. Pons, D. Viganò, U. Geppert, Pulsar timing irregularities and the imprint of magnetic field evolution, A\&A 547 (2012) A9.

[134] N. Andersson, A New Class of Unstable Modes of Rotating Relativistic Stars, ApJ 502 (1998) 708.

[135] N. Andersson, K. Kokkotas, B. F. Schutz, Gravitational Radiation Limit on the Spin of Young Neutron Stars, ApJ 510 (1999) 846-853.

[136] P. Arras, E. E. Flanagan, S. M. Morsink, A. K. Schenk, S. A. Teukolsky, I. Wasserman, Saturation of the r-Mode Instability, ApJ 591 (2003) $1129-1151$.

[137] C. D. Ott, A. Burrows, T. A. Thompson, E. Livne, R. Walder, The Spin Periods and Rotational Profiles of Neutron Stars at Birth, ApJS 164 (2006) 130-155.

[138] H. Tananbaum, H. Gursky, E. M. Kellogg, R. Levinson, E. Schreier, R. Giacconi, Discovery of a Periodic Pulsating Binary X-Ray Source in Hercules from UHURU, ApJL 174 (1972) L143.

[139] J. E. Pringle, M. J. Rees, Accretion Disc Models for Compact X-Ray Sources, A\&A 21 (1972) 1.

[140] A. Davidsen, J. P. Ostriker, The Nature of Cygnus X-3: a Prototype for Old-Population Binary X-Ray Sources, ApJ 189 (1974) 331-338. 
[141] F. K. Lamb, C. J. Pethick, D. Pines, A Model for Compact X-Ray Sources: Accretion by Rotating Magnetic Stars, ApJ 184 (1973) 271290.

[142] P. Ghosh, C. J. Pethick, F. K. Lamb, Accretion by rotating magnetic neutron stars. I - Flow of matter inside the magnetosphere and its implications for spin-up and spin-down of the star, ApJ 217 (1977) $578-596$.

[143] P. Ghosh, F. K. Lamb, Disk accretion by magnetic neutron stars, ApJL 223 (1978) L83-L87.

[144] P. Ghosh, F. K. Lamb, Accretion by rotating magnetic neutron stars. II - Radial and vertical structure of the transition zone in disk accretion, ApJ 232 (1979) 259-276.

[145] P. Ghosh, F. K. Lamb, Accretion by rotating magnetic neutron stars. III - Accretion torques and period changes in pulsating X-ray sources, ApJ 234 (1979) 296-316.

[146] F. Shu, J. Najita, E. Ostriker, F. Wilkin, S. Ruden, S. Lizano, Magnetocentrifugally driven flows from young stars and disks. 1: A generalized model, ApJ 429 (1994) 781-796.

[147] F. H. Shu, J. Najita, S. P. Ruden, S. Lizano, Magnetocentrifugally driven flows from young stars and disks. 2: Formulation of the dynamical problem, ApJ 429 (1994) 797-807.

[148] J. R. Najita, F. H. Shu, Magnetocentrifugally driven flows from young stars and disks. 3: Numerical solution of the sub-Alfvenic region, ApJ 429 (1994) 808-825.

[149] D. C. Backer, S. R. Kulkarni, C. Heiles, M. M. Davis, W. M. Goss, A millisecond pulsar, Nature 300 (1982) 615-618.

[150] V. Radhakrishnan, G. Srinivasan, On the origin of the recently discovered ultra-rapid pulsar, Current Science 51 (1982) 1096-1099.

[151] M. A. Alpar, A. F. Cheng, M. A. Ruderman, J. Shaham, A new class of radio pulsars, Nature 300 (1982) 728-730. 
[152] R. Wijnands, M. van der Klis, A millisecond pulsar in an X-ray binary system, Nature 394 (1998) 344-346.

[153] D. Chakrabarty, E. H. Morgan, The two-hour orbit of a binary millisecond X-ray pulsar, Nature 394 (1998) 346-348.

[154] D. Chakrabarty, E. H. Morgan, M. P. Muno, D. K. Galloway, R. Wijnands, M. van der Klis, C. B. Markwardt, Nuclear-powered millisecond pulsars and the maximum spin frequency of neutron stars, Nature 424 (2003) 42-44.

[155] A. Papitto, C. Ferrigno, E. Bozzo, N. Rea, L. Pavan, L. Burderi, M. Burgay, S. Campana, T. di Salvo, M. Falanga, M. D. Filipović, P. C. C. Freire, J. W. T. Hessels, A. Possenti, S. M. Ransom, A. Riggio, P. Romano, J. M. Sarkissian, I. H. Stairs, L. Stella, D. F. Torres, M. H. Wieringa, G. F. Wong, Swings between rotation and accretion power in a binary millisecond pulsar, Nature 501 (2013) 517-520.

[156] A. Patruno, A. M. Archibald, J. W. T. Hessels, S. Bogdanov, B. W. Stappers, C. G. Bassa, G. H. Janssen, V. M. Kaspi, S. Tendulkar, A. G. Lyne, A New Accretion Disk around the Missing Link Binary System PSR J1023+0038, ApJL 781 (2014) L3.

[157] S. Bogdanov, A. Patruno, A. M. Archibald, C. Bassa, J. W. T. Hessels, G. H. Janssen, B. W. Stappers, X-Ray Observations of XSS J122704859 in a New Low State: A Transformation to a Disk-free Rotationpowered Pulsar Binary, ApJ 789 (2014) 40.

[158] P. Casella, D. Altamirano, A. Patruno, R. Wijnands, M. van der Klis, Discovery of Coherent Millisecond X-Ray Pulsations in Aquila X-1, ApJL 674 (2008) L41-L44.

[159] F. K. Lamb, S. Boutloukos, S. Van Wassenhove, R. T. Chamberlain, K. H. Lo, M. C. Miller, Origin of Intermittent Accretion-Powered Xray Oscillations in Neutron Stars with Millisecond Spin Periods, ApJL 705 (2009) L36-L39.

[160] F. K. Lamb, S. Boutloukos, S. Van Wassenhove, R. T. Chamberlain, K. H. Lo, A. Clare, W. Yu, M. C. Miller, A Model for the Waveform Behavior of Accreting Millisecond X-Ray Pulsars: Nearly Aligned Magnetic Fields and Moving Emission Regions, ApJ 706 (2009) 417-435. 
[161] R. E. Taam, E. P. J. van den Heuvel, Magnetic field decay and the origin of neutron star binaries, ApJ 305 (1986) 235-245.

[162] Z. Arzoumanian, J. M. Cordes, I. Wasserman, Pulsar Spin Evolution, Kinematics, and the Birthrate of Neutron Star Binaries, ApJ 520 (1999) 696-705.

[163] T. M. Tauris, N. Langer, M. Kramer, Formation of millisecond pulsars with CO white dwarf companions - II. Accretion, spin-up, true ages and comparison to MSPs with He white dwarf companions, MNRAS 425 (2012) 1601-1627.

[164] Z. Wang, D. Chakrabarty, D. L. Kaplan, A debris disk around an isolated young neutron star, Nature 440 (2006) 772-775.

[165] T. E. Strohmayer, A. I. Ibrahim, Discovery of a 6.4 KEV Emission Line in a Burst from SGR 1900+14, ApJL 537 (2000) L111-L114.

[166] A. S. Endal, S. Sofia, The evolution of rotating stars. II - Calculations with time-dependent redistribution of angular momentum for 7 - and 10-solar-mass stars, ApJ 220 (1978) 279-290.

[167] J. M. Miller, M. C. Miller, C. S. Reynolds, The Angular Momenta of Neutron Stars and Black Holes as a Window on Supernovae, ApJL 731 (2011) L5.

[168] V. M. Kaspi, D. J. Helfand, Constraining the Birth Events of Neutron Stars, in: P. O. Slane, B. M. Gaensler (Eds.), Neutron Stars in Supernova Remnants, volume 271 of Astronomical Society of the Pacific Conference Series, p. 3.

[169] J. E. Gunn, J. P. Ostriker, On the Nature of Pulsars. III. Analysis of Observations, ApJ 160 (1970) 979.

[170] R. Narayan, J. P. Ostriker, Pulsar populations and their evolution, ApJ 352 (1990) 222-246.

[171] P. L. Gonthier, R. Van Guilder, A. K. Harding, Role of Beam Geometry in Population Statistics and Pulse Profiles of Radio and Gamma-Ray Pulsars, ApJ 604 (2004) 775-790. 
[172] G. Baym, C. Pethick, D. Pikes, Electrical Conductivity of Neutron Star Matter, Nature 224 (1969) 674-675.

[173] P. Goldreich, A. Reisenegger, Magnetic field decay in isolated neutron stars, ApJ 395 (1992) 250-258.

[174] G. M. Stollman, Pulsar statistics, A\&A 178 (1987) 143-152.

[175] D. Bhattacharya, R. A. M. J. Wijers, J. W. Hartman, F. Verbunt, On the decay of the magnetic fields of single radio pulsars, A\&A 254 (1992) 198-212.

[176] D. R. Lorimer, M. Bailes, P. A. Harrison, Pulsar statistics - IV. Pulsar velocities, MNRAS 289 (1997) 592-604.

[177] M. Vivekanand, R. Narayan, A new look at pulsar statistics - Birthrate and evidence for injection, Journal of Astrophysics and Astronomy 2 (1981) 315-337.

[178] D. R. Lorimer, M. Bailes, R. J. Dewey, P. A. Harrison, Pulsar Statistics - the Birthrate and Initial Spin Periods of Radio Pulsars, MNRAS 263 (1993) 403.

[179] R. N. Manchester, A. G. Lyne, F. Camilo, J. F. Bell, V. M. Kaspi, N. D'Amico, N. P. F. McKay, F. Crawford, I. H. Stairs, A. Possenti, M. Kramer, D. C. Sheppard, The Parkes multi-beam pulsar survey I. Observing and data analysis systems, discovery and timing of 100 pulsars, MNRAS 328 (2001) 17-35.

[180] N. Vranesevic, R. N. Manchester, D. R. Lorimer, G. B. Hobbs, A. G. Lyne, M. Kramer, F. Camilo, I. H. Stairs, V. M. Kaspi, N. D'Amico, A. Possenti, F. Crawford, A. J. Faulkner, M. A. McLaughlin, Pulsar Birthrates from the Parkes Multibeam Survey, ApJL 617 (2004) L139L142.

[181] C.-A. Faucher-Giguère, V. M. Kaspi, Birth and Evolution of Isolated Radio Pulsars, ApJ 643 (2006) 332-355.

[182] A. Noutsos, D. H. F. M. Schnitzeler, E. F. Keane, M. Kramer, S. Johnston, Pulsar spin-velocity alignment: kinematic ages, birth periods and braking indices, MNRAS 430 (2013) 2281-2301. 
[183] A. P. Igoshev, S. B. Popov, Neutron star's initial spin period distribution, MNRAS 432 (2013) 967-972.

[184] J. Cottam, F. Paerels, M. Mendez, Gravitationally redshifted absorption lines in the X-ray burst spectra of a neutron star, Nature 420 (2002) 51-54.

[185] J. Cottam, F. Paerels, M. Méndez, L. Boirin, W. H. G. Lewin, E. Kuulkers, J. M. Miller, The Burst Spectra of EXO 0748-676 during a Long 2003 XMM-Newton Observation, ApJ 672 (2008) 504-509.

[186] N. I. Shakura, R. A. Sunyaev, Black holes in binary systems. Observational appearance., A\&A 24 (1973) 337-355.

[187] I. D. Novikov, K. S. Thorne, Astrophysics of black holes., in: C. Dewitt, B. S. Dewitt (Eds.), Black Holes (Les Astres Occlus), pp. 343-450.

[188] J. A. Tomsick, K. Yamaoka, S. Corbel, P. Kaaret, E. Kalemci, S. Migliari, Truncation of the Inner Accretion Disk Around a Black Hole at Low Luminosity, ApJL 707 (2009) L87-L91.

[189] R. C. Reis, A. C. Fabian, J. M. Miller, Black hole accretion discs in the canonical low-hard state, MNRAS 402 (2010) 836-854.

[190] J. M. Miller, G. G. Pooley, A. C. Fabian, M. A. Nowak, R. C. Reis, E. M. Cackett, K. Pottschmidt, J. Wilms, On the Role of the Accretion Disk in Black Hole Disk-Jet Connections, ApJ 757 (2012) 11.

[191] M. T. Reynolds, J. M. Miller, A Swift Survey of Accretion onto Stellarmass Black Holes, ApJ 769 (2013) 16.

[192] A. A. Esin, J. E. McClintock, R. Narayan, Advection-dominated Accretion and the Spectral States of Black Hole X-Ray Binaries: Application to Nova MUSCAE 1991, ApJ 489 (1997) 865.

[193] E. S. Rykoff, J. M. Miller, D. Steeghs, M. A. P. Torres, Swift Observations of the Cooling Accretion Disk of XTE J1817-330, ApJ 666 (2007) 1129-1139.

[194] J. F. Steiner, J. E. McClintock, R. A. Remillard, L. Gou, S. Yamada, R. Narayan, The Constant Inner-disk Radius of LMC X-3: A Basis for Measuring Black Hole Spin, ApJL 718 (2010) L117-L121. 
[195] M. C. Miller, F. K. Lamb, D. Psaltis, Sonic-Point Model of Kilohertz Quasi-periodic Brightness Oscillations in Low-Mass X-Ray Binaries, ApJ 508 (1998) 791-830.

[196] D. Barret, J. Olive, M. C. Miller, An abrupt drop in the coherence of the lower $\mathrm{kHz}$ quasi-periodic oscillations in 4U 1636-536, MNRAS 361 (2005) 855-860.

[197] D. Barret, J. Olive, M. C. Miller, Drop of coherence of the lower kilo$\mathrm{Hz}$ QPO in neutron stars: Is there a link with the innermost stable circular orbit?, Astronomische Nachrichten 326 (2005) 808-811.

[198] D. Barret, J. Olive, M. C. Miller, The coherence of kilohertz quasiperiodic oscillations in the X-rays from accreting neutron stars, MNRAS 370 (2006) 1140-1146.

[199] D. Barret, J. Olive, M. C. Miller, Supporting evidence for the signature of the innermost stable circular orbit in Rossi X-ray data from 4U 1636536, MNRAS 376 (2007) 1139-1144.

[200] M. Méndez, On the maximum amplitude and coherence of the kilohertz quasi-periodic oscillations in low-mass X-ray binaries, MNRAS 371 (2006) 1925-1938.

[201] C. S. Reynolds, M. C. Begelman, Iron Fluorescence from within the Innermost Stable Orbit of Black Hole Accretion Disks, ApJ 488 (1997) 109.

[202] K. A. Sorathia, C. S. Reynolds, P. J. Armitage, Connections Between Local and Global Turbulence in Accretion Disks, ApJ 712 (2010) 12411247 .

[203] J. F. Hawley, X. Guan, J. H. Krolik, Assessing Quantitative Results in Accretion Simulations: From Local to Global, ApJ 738 (2011) 84.

[204] K. A. Sorathia, C. S. Reynolds, J. M. Stone, K. Beckwith, Global Simulations of Accretion Disks. I. Convergence and Comparisons with Local Models, ApJ 749 (2012) 189.

[205] J. F. Hawley, S. A. Richers, X. Guan, J. H. Krolik, Testing Convergence for Global Accretion Disks, ApJ 772 (2013) 102. 
[206] C. S. Reynolds, A. C. Fabian, Broad Iron-K $\alpha$ Emission Lines as a Diagnostic of Black Hole Spin, ApJ 675 (2008) 1048-1056.

[207] R. F. Penna, J. C. McKinney, R. Narayan, A. Tchekhovskoy, R. Shafee, J. E. McClintock, Simulations of magnetized discs around black holes: effects of black hole spin, disc thickness and magnetic field geometry, MNRAS 408 (2010) 752-782.

[208] K. Mitsuda, H. Inoue, K. Koyama, K. Makishima, M. Matsuoka, Y. Ogawara, K. Suzuki, Y. Tanaka, N. Shibazaki, T. Hirano, Energy spectra of low-mass binary X-ray sources observed from TENMA, Pub. Astr. Soc. Japan 36 (1984) 741-759.

[209] S. W. Davis, O. M. Blaes, I. Hubeny, N. J. Turner, Relativistic Accretion Disk Models of High-State Black Hole X-Ray Binary Spectra, ApJ 621 (2005) 372-387.

[210] J. Wambsganss, B. Paczynski, P. Schneider, Interpretation of the microlensing event in QSO 2237 + 0305, ApJL 358 (1990) L33-L36.

[211] K. P. Rauch, R. D. Blandford, Microlensing and the structure of active galactic nucleus accretion disks, ApJL 381 (1991) L39-L42.

[212] D. Pooley, J. A. Blackburne, S. Rappaport, P. L. Schechter, X-Ray and Optical Flux Ratio Anomalies in Quadruply Lensed Quasars. I. Zooming in on Quasar Emission Regions, ApJ 661 (2007) 19-29.

[213] X. Dai, C. S. Kochanek, G. Chartas, S. Kozłowski, C. W. Morgan, G. Garmire, E. Agol, The Sizes of the X-ray and Optical Emission Regions of RXJ 1131-1231, ApJ 709 (2010) 278-285.

[214] C. W. Morgan, C. S. Kochanek, N. D. Morgan, E. E. Falco, The Quasar Accretion Disk Size-Black Hole Mass Relation, ApJ 712 (2010) 1129-1136.

[215] J. A. Blackburne, C. S. Kochanek, The Effect of a Time-varying Accretion Disk Size on Quasar Microlensing Light Curves, ApJ 718 (2010) 1079-1084.

[216] J. Jiménez-Vicente, E. Mediavilla, J. A. Muñoz, C. S. Kochanek, A Robust Determination of the Size of Quasar Accretion Disks Using Gravitational Microlensing, ApJ 751 (2012) 106. 
[217] J. Jiménez-Vicente, E. Mediavilla, C. S. Kochanek, J. A. Muñoz, V. Motta, E. Falco, A. M. Mosquera, The Average Size and Temperature Profile of Quasar Accretion Disks, ApJ 783 (2014) 47.

[218] J. Dexter, E. Agol, Quasar Accretion Disks are Strongly Inhomogeneous, ApJL 727 (2011) L24.

[219] J. Dexter, E. Quataert, Inhomogeneous accretion discs and the soft states of black hole X-ray binaries, MNRAS 426 (2012) L71-L75.

[220] J. J. Ruan, S. F. Anderson, J. Dexter, E. Agol, Evidence for Large Temperature Fluctuations in Quasar Accretion Disks from Spectral Variability, ApJ 783 (2014) 105.

[221] Y.-F. Jiang, J. M. Stone, S. W. Davis, On the Thermal Stability of Radiation-dominated Accretion Disks, ApJ 778 (2013) 65.

[222] S. W. Davis, C. Done, O. M. Blaes, Testing Accretion Disk Theory in Black Hole X-Ray Binaries, ApJ 647 (2006) 525-538.

[223] T. J. Maccarone, On the misalignment of jets in microquasars, MNRAS 336 (2002) 1371-1376.

[224] R. G. Martin, C. A. Tout, J. E. Pringle, Alignment time-scale of the microquasar GRO J1655-40, MNRAS 387 (2008) 188-196.

[225] J. F. Steiner, J. E. McClintock, Modeling the Jet Kinematics of the Black Hole Microquasar XTE J1550-564: A Constraint on Spin-Orbit Alignment, ApJ 745 (2012) 136.

[226] R. M. Hjellming, M. P. Rupen, Episodic ejection of relativistic jets by the X-ray transient GRO J1655 - 40, Nature 375 (1995) 464-468.

[227] J. A. Orosz, E. Kuulkers, M. van der Klis, J. E. McClintock, M. R. Garcia, P. J. Callanan, C. D. Bailyn, R. K. Jain, R. A. Remillard, A Black Hole in the Superluminal Source SAX J1819.3-2525 (V4641 Sgr), ApJ 555 (2001) 489-503.

[228] P. C. Fragile, G. J. Mathews, J. R. Wilson, Bardeen-Petterson Effect and Quasi-periodic Oscillations in X-Ray Binaries, ApJ 553 (2001) 955-959. 
[229] A. Ingram, C. Done, P. C. Fragile, Low-frequency quasi-periodic oscillations spectra and Lense-Thirring precession, MNRAS 397 (2009) L101-L105.

[230] J. Dexter, P. C. Fragile, Observational Signatures of Tilted Black Hole Accretion Disks from Simulations, ApJ 730 (2011) 36.

[231] T. R. White, A. P. Lightman, A. A. Zdziarski, Compton reflection of gamma rays by cold electrons, ApJ 331 (1988) 939-948.

[232] I. M. George, A. C. Fabian, X-ray reflection from cold matter in active galactic nuclei and X-ray binaries, MNRAS 249 (1991) 352-367.

[233] M. G. Revnivtsev, E. M. Churazov, S. Y. Sazonov, R. A. Sunyaev, A. A. Lutovinov, M. R. Gilfanov, A. A. Vikhlinin, P. E. Shtykovsky, M. N. Pavlinsky, Hard X-ray view of the past activity of Sgr A* in a natural Compton mirror, A\&A 425 (2004) L49-L52.

[234] X. W. Shu, T. Yaqoob, J. X. Wang, The Cores of the Fe K $\alpha$ Lines in Active Galactic Nuclei: An Extended Chandra High Energy Grating Sample, ApJS 187 (2010) 581-606.

[235] X. W. Shu, T. Yaqoob, J. X. Wang, Chandra High-energy Grating Observations of the Fe K $\alpha$ Line Core in Type II Seyfert Galaxies: A Comparison with Type I Nuclei, ApJ 738 (2011) 147.

[236] K. Nandra, P. M. O’Neill, I. M. George, J. N. Reeves, An XMMNewton survey of broad iron lines in Seyfert galaxies, MNRAS 382 (2007) 194-228.

[237] R. R. Ross, A. C. Fabian, A comprehensive range of X-ray ionizedreflection models, MNRAS 358 (2005) 211-216.

[238] J. M. Miller, M. L. Parker, F. Fuerst, M. Bachetti, F. A. Harrison, D. Barret, S. E. Boggs, D. Chakrabarty, F. E. Christensen, W. W. Craig, A. C. Fabian, B. W. Grefenstette, C. J. Hailey, A. L. King, D. K. Stern, J. A. Tomsick, D. J. Walton, W. W. Zhang, NuSTAR Spectroscopy of GRS 1915+105: Disk Reflection, Spin, and Connections to Jets, ApJL 775 (2013) L45. 
[239] J. García, T. R. Kallman, X-ray Reflected Spectra from Accretion Disk Models. I. Constant Density Atmospheres, ApJ 718 (2010) 695-706.

[240] J. García, T. R. Kallman, R. F. Mushotzky, X-ray Reflected Spectra from Accretion Disk Models. II. Diagnostic Tools for X-ray Observations, ApJ 731 (2011) 131.

[241] J. García, T. Dauser, C. S. Reynolds, T. R. Kallman, J. E. McClintock, J. Wilms, W. Eikmann, X-Ray Reflected Spectra from Accretion Disk Models. III. A Complete Grid of Ionized Reflection Calculations, ApJ 768 (2013) 146.

[242] J. García, T. Dauser, A. Lohfink, T. R. Kallman, J. F. Steiner, J. E. McClintock, L. Brenneman, J. Wilms, W. Eikmann, C. S. Reynolds, F. Tombesi, Improved Reflection Models of Black Hole Accretion Disks: Treating the Angular Distribution of X-Rays, ApJ 782 (2014) 76.

[243] G. Miniutti, A. C. Fabian, A light bending model for the X-ray temporal and spectral properties of accreting black holes, MNRAS 349 (2004) 1435-1448.

[244] G. Miniutti, A. C. Fabian, J. M. Miller, The relativistic Fe emission line in XTE J1650-500 with BeppoSAX: evidence for black hole spin and light-bending effects?, MNRAS 351 (2004) 466-472.

[245] S. Rossi, J. Homan, J. M. Miller, T. Belloni, Iron-line and continuum flux variations in the RXTE spectra of the black hole candidate XTE J1650-500, MNRAS 360 (2005) 763-768.

[246] R. C. Reis, J. M. Miller, M. T. Reynolds, A. C. Fabian, D. J. Walton, E. Cackett, J. F. Steiner, Evidence of Light-bending Effects and Its Implication for Spectral State Transitions, ApJ 763 (2013) 48.

[247] A. C. Fabian, M. J. Rees, L. Stella, N. E. White, X-ray fluorescence from the inner disc in Cygnus X-1, MNRAS 238 (1989) 729-736.

[248] A. Laor, Line profiles from a disk around a rotating black hole, ApJ 376 (1991) 90-94.

[249] M. Dovčiak, V. Karas, T. Yaqoob, An Extended Scheme for Fitting XRay Data with Accretion Disk Spectra in the Strong Gravity Regime, ApJS 153 (2004) 205-221. 
[250] K. Beckwith, C. Done, Iron line profiles in strong gravity, MNRAS 352 (2004) 353-362.

[251] L. W. Brenneman, C. S. Reynolds, Constraining Black Hole Spin via X-Ray Spectroscopy, ApJ 652 (2006) 1028-1043.

[252] T. Dauser, J. Garcia, J. Wilms, M. Bock, L. W. Brenneman, M. Falanga, K. Fukumura, C. S. Reynolds, Irradiation of an accretion disc by a jet: general properties and implications for spin measurements of black holes, MNRAS 430 (2013) 1694-1708.

[253] J. M. Miller, Relativistic X-Ray Lines from the Inner Accretion Disks Around Black Holes, Ann. Rev. Astron. Astrophys. 45 (2007) 441-479.

[254] A. C. Fabian, A. Zoghbi, R. R. Ross, P. Uttley, L. C. Gallo, W. N. Brandt, A. J. Blustin, T. Boller, M. D. Caballero-Garcia, J. Larsson, J. M. Miller, G. Miniutti, G. Ponti, R. C. Reis, C. S. Reynolds, Y. Tanaka, A. J. Young, Broad line emission from iron K- and Lshell transitions in the active galaxy 1H0707-495, Nature 459 (2009) $540-542$.

[255] D. R. Wilkins, A. C. Fabian, Understanding X-ray reflection emissivity profiles in AGN: locating the X-ray source, MNRAS 424 (2012) 12841296.

[256] D. A. Liedahl, D. F. Torres, Atomic X-ray spectroscopy of accreting black holes, Canadian Journal of Physics 83 (2005) 1177-1240.

[257] J. M. Miller, C. S. Reynolds, A. C. Fabian, G. Miniutti, L. C. Gallo, Stellar-Mass Black Hole Spin Constraints from Disk Reflection and Continuum Modeling, ApJ 697 (2009) 900-912.

[258] B. Hiemstra, M. Méndez, C. Done, M. Díaz Trigo, D. Altamirano, P. Casella, A strong and broad Fe line in the XMM-Newton spectrum of the new X-ray transient and black hole candidate XTE J1652-453, MNRAS 411 (2011) 137-150.

[259] R. C. Reis, J. M. Miller, A. C. Fabian, E. M. Cackett, D. Maitra, C. S. Reynolds, M. Rupen, D. T. H. Steeghs, R. Wijnands, Multistate observations of the Galactic black hole XTE J1752-223: evidence for an intermediate black hole spin, MNRAS 410 (2011) 2497-2505. 
[260] A. L. King, J. M. Miller, K. Gültekin, D. J. Walton, A. C. Fabian, C. S. Reynolds, K. Nandra, What is on Tap? The Role of Spin in Compact Objects and Relativistic Jets, ApJ 771 (2013) 84.

[261] S. C. Noble, J. H. Krolik, J. D. Schnittman, J. F. Hawley, Radiative Efficiency and Thermal Spectrum of Accretion onto Schwarzschild Black Holes, ApJ 743 (2011) 115.

[262] J. D. Schnittman, J. H. Krolik, S. C. Noble, X-Ray Spectra from Magnetohydrodynamic Simulations of Accreting Black Holes, ApJ 769 (2013) 156.

[263] R. C. Reis, J. M. Miller, On the Size and Location of the X-Ray Emitting Coronae around Black Holes, ApJL 769 (2013) L7.

[264] G. Chartas, C. S. Kochanek, X. Dai, S. Poindexter, G. Garmire, XRay Microlensing in RXJ1131-1231 and HE1104-1805, ApJ 693 (2009) 174-185.

[265] J. Wilms, C. S. Reynolds, M. C. Begelman, J. Reeves, S. Molendi, R. Staubert, E. Kendziorra, XMM-EPIC observation of MCG-6-30-15: direct evidence for the extraction of energy from a spinning black hole?, MNRAS 328 (2001) L27-L31.

[266] K. Beckwith, J. F. Hawley, J. H. Krolik, Where is the radiation edge in magnetized black hole accretion discs?, MNRAS 390 (2008) 21-38.

[267] S. C. Noble, J. H. Krolik, J. F. Hawley, Direct Calculation of the Radiative Efficiency of an Accretion Disk Around a Black Hole, ApJ 692 (2009) 411-421.

[268] A. K. Kulkarni, R. F. Penna, R. V. Shcherbakov, J. F. Steiner, R. Narayan, A. Sä Dowski, Y. Zhu, J. E. McClintock, S. W. Davis, J. C. McKinney, Measuring black hole spin by the continuum-fitting method: effect of deviations from the Novikov-Thorne disc model, MNRAS 414 (2011) 1183-1194.

[269] V. Dhawan, I. F. Mirabel, L. F. Rodríguez, AU-Scale Synchrotron Jets and Superluminal Ejecta in GRS 1915+105, ApJ 543 (2000) 373-385. 
[270] R. D. Blandford, R. L. Znajek, Electromagnetic extraction of energy from Kerr black holes, MNRAS 179 (1977) 433-456.

[271] J. M. Miller, A. C. Fabian, M. A. Nowak, W. H. G. Lewin, Relativistic Iron Lines in Galactic Black Holes:. Recent Results and Lines in the ASCA Archive, in: M. Novello, S. Perez Bergliaffa, R. Ruffini (Eds.), The Tenth Marcel Grossmann Meeting. Proceedings of the MG10 Meeting held at Brazilian Center for Research in Physics (CBPF), Rio de Janeiro, Brazil, 20-26 July 2003, Eds.: Mário Novello; Santiago Perez Bergliaffa; Remo Ruffini. Singapore: World Scientific Publishing, in 3 volumes, ISBN 981-256-667-8 (set), ISBN 981-256-980-4 (Part A), ISBN 981-256-979-0 (Part B), ISBN 981-256-978-2 (Part C), 2006, XLVIII + 2492 pp.: 2006, p.1296, p. 1296.

[272] A. Martocchia, G. Matt, V. Karas, T. Belloni, M. Feroci, Evidence for a relativistic iron line in GRS 1915+105, A\&A 387 (2002) 215-221.

[273] J. L. Blum, J. M. Miller, A. C. Fabian, M. C. Miller, J. Homan, M. van der Klis, E. M. Cackett, R. C. Reis, Measuring the Spin of GRS 1915+105 with Relativistic Disk Reflection, ApJ 706 (2009) 60-66.

[274] F. A. Harrison, W. W. Craig, F. E. Christensen, C. J. Hailey, W. W. Zhang, S. E. Boggs, D. Stern, W. R. Cook, K. Forster, P. Giommi, B. W. Grefenstette, Y. Kim, T. Kitaguchi, J. E. Koglin, K. K. Madsen, P. H. Mao, H. Miyasaka, K. Mori, M. Perri, M. J. Pivovaroff, S. Puccetti, V. R. Rana, N. J. Westergaard, J. Willis, A. Zoglauer, H. An, M. Bachetti, N. M. Barrière, E. C. Bellm, V. Bhalerao, N. F. Brejnholt, F. Fuerst, C. C. Liebe, C. B. Markwardt, M. Nynka, J. K. Vogel, D. J. Walton, D. R. Wik, D. M. Alexander, L. R. Cominsky, A. E. Hornschemeier, A. Hornstrup, V. M. Kaspi, G. M. Madejski, G. Matt, S. Molendi, D. M. Smith, J. A. Tomsick, M. Ajello, D. R. Ballantyne, M. Baloković, D. Barret, F. E. Bauer, R. D. Blandford, W. N. Brandt, L. W. Brenneman, J. Chiang, D. Chakrabarty, J. Chenevez, A. Comastri, F. Dufour, M. Elvis, A. C. Fabian, D. Farrah, C. L. Fryer, E. V. Gotthelf, J. E. Grindlay, D. J. Helfand, R. Krivonos, D. L. Meier, J. M. Miller, L. Natalucci, P. Ogle, E. O. Ofek, A. Ptak, S. P. Reynolds, J. R. Rigby, G. Tagliaferri, S. E. Thorsett, E. Treister, C. M. Urry, The Nuclear Spectroscopic Telescope Array (NuSTAR) High-energy X-Ray Mission, ApJ 770 (2013) 103. 
[275] J. E. McClintock, R. Shafee, R. Narayan, R. A. Remillard, S. W. Davis, L.-X. Li, The Spin of the Near-Extreme Kerr Black Hole GRS 1915+105, ApJ 652 (2006) 518-539.

[276] J. M. Miller, A. C. Fabian, R. Wijnands, C. S. Reynolds, M. Ehle, M. J. Freyberg, M. van der Klis, W. H. G. Lewin, C. Sanchez-Fernandez, A. J. Castro-Tirado, Evidence of Spin and Energy Extraction in a Galactic Black Hole Candidate: The XMM-Newton/EPIC-pn Spectrum of XTE J1650-500, ApJL 570 (2002) L69-L73.

[277] J. M. Miller, A. C. Fabian, C. S. Reynolds, M. A. Nowak, J. Homan, M. J. Freyberg, M. Ehle, T. Belloni, R. Wijnands, M. van der Klis, P. A. Charles, W. H. G. Lewin, Evidence of Black Hole Spin in GX 339-4: XMM-Newton/EPIC-pn and RXTE Spectroscopy of the Very High State, ApJL 606 (2004) L131-L134.

[278] J. M. Miller, C. S. Reynolds, A. C. Fabian, E. M. Cackett, G. Miniutti, J. Raymond, D. Steeghs, R. Reis, J. Homan, Initial Measurements of Black Hole Spin in GX 339-4 from Suzaku Spectroscopy, ApJL 679 (2008) L113-L116.

[279] R. C. Reis, A. C. Fabian, R. R. Ross, G. Miniutti, J. M. Miller, C. Reynolds, A systematic look at the very high and low/hard state of GX339-4: constraining the black hole spin with a new reflection model, MNRAS 387 (2008) 1489-1498.

[280] S. Bhattacharyya, T. E. Strohmayer, Evidence of a Broad Relativistic Iron Line from the Neutron Star Low-Mass X-Ray Binary Serpens X-1, ApJL 664 (2007) L103-L106.

[281] E. M. Cackett, J. M. Miller, S. Bhattacharyya, J. E. Grindlay, J. Homan, M. van der Klis, M. C. Miller, T. E. Strohmayer, R. Wijnands, Relativistic Iron Emission Lines in Neutron Star Low-Mass X-Ray Binaries as Probes of Neutron Star Radii, ApJ 674 (2008) 415420.

[282] E. M. Cackett, J. M. Miller, D. R. Ballantyne, D. Barret, S. Bhattacharyya, M. Boutelier, M. C. Miller, T. E. Strohmayer, R. Wijnands, Relativistic Lines and Reflection from the Inner Accretion Disks Around Neutron Stars, ApJ 720 (2010) 205-225. 
[283] J. M. Miller, M. L. Parker, F. Fuerst, M. Bachetti, D. Barret, B. W. Grefenstette, S. Tendulkar, F. A. Harrison, S. E. Boggs, D. Chakrabarty, F. E. Christensen, W. W. Craig, A. C. Fabian, C. J. Hailey, L. Natalucci, F. Paerels, V. Rana, D. K. Stern, J. A. Tomsick, W. W. Zhang, Constraints on the Neutron Star and Inner Accretion Flow in Serpens X-1 Using NuSTAR, ApJL 779 (2013) L2.

[284] J. B. Hartle, K. S. Thorne, Slowly Rotating Relativistic Stars. II. Models for Neutron Stars and Supermassive Stars, ApJ 153 (1968) 807.

[285] M. C. Miller, F. K. Lamb, G. B. Cook, Effects of Rapid Stellar Rotation on Equation-of-State Constraints Derived from Quasi-periodic Brightness Oscillations, ApJ 509 (1998) 793-801.

[286] S. N. Zhang, W. Cui, W. Chen, Black Hole Spin in X-Ray Binaries: Observational Consequences, ApJL 482 (1997) L155-L158.

[287] T. Shimura, F. Takahara, On the spectral hardening factor of the Xray emission from accretion disks in black hole candidates, ApJ 445 (1995) 780-788.

[288] A. Merloni, A. C. Fabian, R. R. Ross, On the interpretation of the multicolour disc model for black hole candidates, MNRAS 313 (2000) 193-197.

[289] L.-X. Li, E. R. Zimmerman, R. Narayan, J. E. McClintock, Multitemperature Blackbody Spectrum of a Thin Accretion Disk around a Kerr Black Hole: Model Computations and Comparison with Observations, ApJS 157 (2005) 335-370.

[290] J. C. A. Miller-Jones, P. G. Jonker, V. Dhawan, W. Brisken, M. P. Rupen, G. Nelemans, E. Gallo, The First Accurate Parallax Distance to a Black Hole, ApJL 706 (2009) L230-L234.

[291] M. J. Reid, J. E. McClintock, R. Narayan, L. Gou, R. A. Remillard, J. A. Orosz, The Trigonometric Parallax of Cygnus X-1, ApJ 742 (2011) 83.

[292] J. E. McClintock, R. A. Remillard, Black hole binaries, pp. 157-213. 
[293] D. Steeghs, J. E. McClintock, S. G. Parsons, M. J. Reid, S. Littlefair, V. S. Dhillon, The Not-so-massive Black Hole in the Microquasar GRS1915+105, ApJ 768 (2013) 185.

[294] R. P. Fender, S. T. Garrington, D. J. McKay, T. W. B. Muxlow, G. G. Pooley, R. E. Spencer, A. M. Stirling, E. B. Waltman, MERLIN observations of relativistic ejections from GRS 1915+105, MNRAS 304 (1999) 865-876.

[295] M. Middleton, C. Done, M. Gierliński, S. W. Davis, Black hole spin in GRS 1915+105, MNRAS 373 (2006) 1004-1012.

[296] L. Gou, J. E. McClintock, M. J. Reid, J. A. Orosz, J. F. Steiner, R. Narayan, J. Xiang, R. A. Remillard, K. A. Arnaud, S. W. Davis, The Extreme Spin of the Black Hole in Cygnus X-1, ApJ 742 (2011) 85 .

[297] A. C. Fabian, D. R. Wilkins, J. M. Miller, R. C. Reis, C. S. Reynolds, E. M. Cackett, M. A. Nowak, G. G. Pooley, K. Pottschmidt, J. S. Sanders, R. R. Ross, J. Wilms, On the determination of the spin of the black hole in Cyg X-1 from X-ray reflection spectra, MNRAS 424 (2012) 217-223.

[298] J. A. Tomsick, M. A. Nowak, M. Parker, J. M. Miller, A. C. Fabian, F. A. Harrison, M. Bachetti, D. Barret, S. E. Boggs, F. E. Christensen, W. W. Craig, K. Forster, F. Fürst, B. W. Grefenstette, C. J. Hailey, A. L. King, K. K. Madsen, L. Natalucci, K. Pottschmidt, R. R. Ross, D. Stern, D. J. Walton, J. Wilms, W. W. Zhang, The Reflection Component from Cygnus X-1 in the Soft State Measured by NuSTAR and Suzaku, ApJ 780 (2014) 78.

[299] J. A. Orosz, J. E. McClintock, J. P. Aufdenberg, R. A. Remillard, M. J. Reid, R. Narayan, L. Gou, The Mass of the Black Hole in Cygnus X-1, ApJ 742 (2011) 84.

[300] J. Ziółkowski, Determination of the masses of the components of the HDE 226868/Cyg X-1 binary system, MNRAS (2014).

[301] J. M. Miller, A. D'Aì, M. W. Bautz, S. Bhattacharyya, D. N. Burrows, E. M. Cackett, A. C. Fabian, M. J. Freyberg, F. Haberl, J. Kennea, M. A. Nowak, R. C. Reis, T. E. Strohmayer, M. Tsujimoto, On 
Relativistic Disk Spectroscopy in Compact Objects with X-ray CCD Cameras, ApJ 724 (2010) 1441-1455.

[302] J. Liu, J. E. McClintock, R. Narayan, S. W. Davis, J. A. Orosz, Precise Measurement of the Spin Parameter of the Stellar-Mass Black Hole M33 X-7, ApJL 679 (2008) L37-L40.

[303] J. Liu, J. E. McClintock, R. Narayan, S. W. Davis, J. A. Orosz, Erratum: "Precise Measurement of the Spin Parameter of the Stellar-mass Black Hole M33 X-7", ApJL 719 (2010) L109.

[304] J. A. Orosz, J. F. Steiner, J. E. McClintock, M. M. Buxton, C. D. Bailyn, D. Steeghs, A. Guberman, M. A. P. Torres, The Mass of the Black Hole in LMC X-3, ArXiv e-prints (2014).

[305] J. F. Steiner, J. E. McClintock, J. A. Orosz, R. A. Remillard, C. D. Bailyn, M. Kolehmainen, O. Straub, The Low-Spin Black Hole in LMC X-3, arXiv:1402.0148 (2014).

[306] C. S. Reynolds, M. C. Miller, The Time Variability of Geometrically Thin Black Hole Accretion Disks. I. The Search for Modes in Simulated Disks, ApJ 692 (2009) 869-886.

[307] T. M. Belloni, A. Sanna, M. Méndez, High-frequency quasi-periodic oscillations in black hole binaries, MNRAS 426 (2012) 1701-1709.

[308] T. M. Belloni, D. Altamirano, Discovery of a $34 \mathrm{~Hz}$ quasi-periodic oscillation in the X-ray emission of GRS 1915+105, MNRAS 432 (2013) $19-22$.

[309] J. Dexter, O. Blaes, A model of the steep power-law spectra and high-frequency quasi-periodic oscillations in luminous black hole X-ray binaries, MNRAS 438 (2014) 3352-3357.

[310] J. Homan, R. Wijnands, M. van der Klis, T. Belloni, J. van Paradijs, M. Klein-Wolt, R. Fender, M. Méndez, Correlated X-Ray Spectral and Timing Behavior of the Black Hole Candidate XTE J1550-564: A New Interpretation of Black Hole States, ApJS 132 (2001) 377-402.

[311] J. M. Miller, R. Wijnands, J. Homan, T. Belloni, D. Pooley, S. Corbel, C. Kouveliotou, M. van der Klis, W. H. G. Lewin, High-Frequency 
Quasi-Periodic Oscillations in the 2000 Outburst of the Galactic Microquasar XTE J1550-564, ApJ 563 (2001) 928-933.

[312] M. van der Klis, Rapid X-ray Variability, pp. 39-112.

[313] T. E. Strohmayer, Discovery of a 450 HZ Quasi-periodic Oscillation from the Microquasar GRO J1655-40 with the Rossi X-Ray Timing Explorer, ApJL 552 (2001) L49-L53.

[314] J. C. McKinney, A. Tchekhovskoy, R. D. Blandford, General relativistic magnetohydrodynamic simulations of magnetically choked accretion flows around black holes, MNRAS 423 (2012) 3083-3117.

[315] L. Stella, M. Vietri, S. M. Morsink, Correlations in the Quasi-periodic Oscillation Frequencies of Low-Mass X-Ray Binaries and the Relativistic Precession Model, ApJL 524 (1999) L63-L66.

[316] S. E. Motta, T. M. Belloni, L. Stella, T. Muñoz-Darias, R. Fender, Precise mass and spin measurements for a stellar-mass black hole through X-ray timing: the case of GRO J1655-40, MNRAS 437 (2014) 25542565.

[317] C. A. Perez, A. S. Silbergleit, R. V. Wagoner, D. E. Lehr, Relativistic Diskoseismology. I. Analytical Results for "Gravity Modes", ApJ 476 (1997) 589.

[318] M. A. Nowak, R. V. Wagoner, M. C. Begelman, D. E. Lehr, The 67 HZ Feature in the Black Hole Candidate GRS $1915+105$ as a Possible "Diskoseismic" Mode, ApJL 477 (1997) L91.

[319] Y. Kato, M. R. Hayashi, S. Miyaji, R. Matsumoto, Magnetohydrodynamic simulations of accretion disks around a weakly magnetized neutron star in strong gravity, Advances in Space Research 28 (2001) $505-510$.

[320] R. V. Wagoner, Relativistic and Newtonian diskoseismology, New Astronomy 51 (2008) 828-834.

[321] R. V. Wagoner, Diskoseismology and QPOs Confront Black Hole Spin, ApJL 752 (2012) L18. 
[322] D. Barret, K. Nandra, X. Barcons, A. Fabian, J. W. den Herder, L. Piro, M. Watson, J. Aird, G. Branduardi-Raymont, M. Cappi, F. Carrera, A. Comastri, E. Costantini, J. Croston, A. Decourchelle, C. Done, M. Dovciak, S. Ettori, A. Finoguenov, A. Georgakakis, P. Jonker, J. Kaastra, G. Matt, C. Motch, P. O'Brien, G. Pareschi, E. Pointecouteau, G. Pratt, G. Rauw, T. Reiprich, J. Sanders, S. Sciortino, R. Willingale, J. Wilms, Athenat: The first Deep Universe X-ray Observatory, in: L. Cambresy, F. Martins, E. Nuss, A. Palacios (Eds.), SF2A-2013: Proceedings of the Annual meeting of the French Society of Astronomy and Astrophysics, pp. 447-453.

[323] e. a. Feroci, M., The Large Observatory for X-ray Timing (LOFT), Experimental Astronomy 34 (2012) 415-444.

[324] J. Homan, M. Klein-Wolt, S. Rossi, J. M. Miller, R. Wijnands, T. Belloni, M. van der Klis, W. H. G. Lewin, High-Frequency Quasi-periodic Oscillations in the Black Hole X-Ray Transient XTE J1650-500, ApJ 586 (2003) 1262-1267.

[325] J. Homan, J. M. Miller, R. Wijnands, M. van der Klis, T. Belloni, D. Steeghs, W. H. G. Lewin, High- and Low-Frequency Quasi-periodic Oscillations in the X-Ray Light Curves of the Black Hole Transient H1743-322, ApJ 623 (2005) 383-391.

[326] M. Klein-Wolt, J. Homan, M. van der Klis, High frequency features in the 1998 outburst of $4 \mathrm{U}$ 1630-47, Nuclear Physics B Proceedings Supplements 132 (2004) 381-386.

[327] J. D. Schnittman, J. Homan, J. M. Miller, A Precessing Ring Model for Low-Frequency Quasi-periodic Oscillations, ApJ 642 (2006) 420-426.

[328] D. Altamirano, T. Belloni, Discovery of High-frequency Quasi-periodic Oscillations in the Black Hole Candidate IGR J17091-3624, ApJL 747 (2012) L4.

[329] E. H. Morgan, R. A. Remillard, J. Greiner, RXTE Observations of QPOs in the Black Hole Candidate GRS 1915+105, ApJ 482 (1997) 993.

[330] J. D. Schnittman, J. H. Krolik, X-ray Polarization from Accreting Black Holes: The Thermal State, ApJ 701 (2009) 1175-1187. 
[331] S. W. Davis, O. M. Blaes, S. Hirose, J. H. Krolik, The Effects of Magnetic Fields and Inhomogeneities on Accretion Disk Spectra and Polarization, ApJ 703 (2009) 569-584.

[332] P. A. Connors, R. F. Stark, T. Piran, Polarization features of X-ray radiation emitted near black holes, ApJ 235 (1980) 224-244.

[333] L.-X. Li, R. Narayan, J. E. McClintock, Inferring the Inclination of a Black Hole Accretion Disk from Observations of its Polarized Continuum Radiation, ApJ 691 (2009) 847-865.

[334] J. D. Schnittman, J. H. Krolik, X-ray Polarization from Accreting Black Holes: Coronal Emission, ApJ 712 (2010) 908-924.

[335] H. Krawczynski, A. Garson, Q. Guo, M. G. Baring, P. Ghosh, M. Beilicke, K. Lee, Scientific prospects for hard X-ray polarimetry, Astroparticle Physics 34 (2011) 550-567.

[336] M. Beilicke, M. G. Baring, S. Barthelmy, W. R. Binns, J. Buckley, R. Cowsik, P. Dowkontt, A. Garson, Q. Guo, Y. Haba, M. H. Israel, H. Kunieda, K. Lee, H. Matsumoto, T. Miyazawa, T. Okajima, J. Schnittman, K. Tamura, J. Tueller, H. Krawczynski, Design and tests of the hard x-ray polarimeter X-Calibur, in: Society of PhotoOptical Instrumentation Engineers (SPIE) Conference Series, volume 8145 of Society of Photo-Optical Instrumentation Engineers (SPIE) Conference Series.

[337] K. Jahoda, The Gravity and Extreme Magnetism Small Explorer, in: Society of Photo-Optical Instrumentation Engineers (SPIE) Conference Series, volume 7732 of Society of Photo-Optical Instrumentation Engineers (SPIE) Conference Series.

[338] J. Schnittman, L. Angelini, M. Baring, W. Baumgartner, K. Black, J. Dotson, P. Ghosh, A. Harding, J. Hill, K. Jahoda, P. Kaaret, T. Kallman, H. Krawczynski, J. Krolik, D. Lai, C. Markwardt, H. Marshall, J. Martoff, R. Morris, T. Okajima, R. Petre, J. Poutanen, S. Reynolds, J. Scargle, P. Serlemitsos, Y. Soong, T. Strohmayer, J. Swank, Y. Tawara, T. Tamagawa, X-ray Polarization from Black Holes: GEMS Scientific White Paper, arXiv:1301.1957 (2013). 
[339] E. Berti, M. Volonteri, Cosmological Black Hole Spin Evolution by Mergers and Accretion, ApJ 684 (2008) 822-828.

[340] C. S. Reynolds, Measuring Black Hole Spin Using X-Ray Reflection Spectroscopy, Space Sci. Rev. (2013).

[341] C. S. Reynolds, The spin of supermassive black holes, Classical and Quantum Gravity 30 (2013) 244004.

[342] R. C. Reis, M. T. Reynolds, J. M. Miller, D. J. Walton, Reflection from the strong gravity regime in a lensed quasar at redshift $\mathrm{z}=0.658$, Nature 507 (2014) 207-209.

[343] J. F. Steiner, R. C. Reis, A. C. Fabian, R. A. Remillard, J. E. McClintock, L. Gou, R. Cooke, L. W. Brenneman, J. S. Sanders, A broad iron line in LMC X-1, MNRAS 427 (2012) 2552-2561.

[344] L. Gou, J. E. McClintock, J. Liu, R. Narayan, J. F. Steiner, R. A. Remillard, J. A. Orosz, S. W. Davis, K. Ebisawa, E. M. Schlegel, A Determination of the Spin of the Black Hole Primary in LMC X-1, ApJ 701 (2009) 1076-1090.

[345] L. Gou, J. E. McClintock, J. F. Steiner, R. Narayan, A. G. Cantrell, C. D. Bailyn, J. A. Orosz, The Spin of the Black Hole in the Soft X-ray Transient A0620-00, ApJL 718 (2010) L122-L126.

[346] W. R. Morningstar, J. M. Miller, R. C. Reis, K. Ebisawa, The Spin of the Black Hole GS 1124-683: Observation of a Retrograde Accretion Disk?, ApJL 784 (2014) L18.

[347] R. Shafee, J. E. McClintock, R. Narayan, S. W. Davis, L.-X. Li, R. A. Remillard, Estimating the Spin of Stellar-Mass Black Holes by Spectral Fitting of the X-Ray Continuum, ApJL 636 (2006) L113-L116.

[348] J. F. Steiner, R. C. Reis, J. E. McClintock, R. Narayan, R. A. Remillard, J. A. Orosz, L. Gou, A. C. Fabian, M. A. P. Torres, The spin of the black hole microquasar XTE J1550-564 via the continuum-fitting and Fe-line methods, MNRAS 416 (2011) 941-958.

[349] A. L. King, D. J. Walton, J. M. Miller, D. Barret, S. E. Boggs, F. E. Christensen, W. W. Craig, A. C. Fabian, F. Fürst, C. J. Hailey, F. A. 
Harrison, R. Krivonos, K. Mori, L. Natalucci, D. Stern, J. A. Tomsick, W. W. Zhang, The Disk Wind in the Rapidly Spinning Stellar-mass Black Hole 4U 1630-472 Observed with NuSTAR, ApJL 784 (2014) L2.

[350] R. C. Reis, A. C. Fabian, R. R. Ross, J. M. Miller, Determining the spin of two stellar-mass black holes from disc reflection signatures, MNRAS 395 (2009) 1257-1264.

[351] R. C. Reis, J. M. Miller, M. T. Reynolds, A. C. Fabian, D. J. Walton, Suzaku Observation of the Black Hole Candidate Maxi J1836-194 in a Hard/Intermediate Spectral State, ApJ 751 (2012) 34.

[352] R. C. Reis, M. T. Reynolds, J. M. Miller, D. J. Walton, D. Maitra, A. King, N. Degenaar, SWIFT J1910.2-0546: A Possible Black Hole Binary with a Retrograde Spin or Truncated Disk, ApJ 778 (2013) 155.

[353] K. Yagi, N. Yunes, I-Love-Q relations in neutron stars and their applications to astrophysics, gravitational waves, and fundamental physics, Phys. Rev. D 88 (2013) 023009.

[354] J. A. Orosz, J. E. McClintock, R. Narayan, C. D. Bailyn, J. D. Hartman, L. Macri, J. Liu, W. Pietsch, R. A. Remillard, A. Shporer, T. Mazeh, A 15.65-solar-mass black hole in an eclipsing binary in the nearby spiral galaxy M 33, Nature 449 (2007) 872-875.

[355] J. A. Orosz, D. Steeghs, J. E. McClintock, M. A. P. Torres, I. Bochkov, L. Gou, R. Narayan, M. Blaschak, A. M. Levine, R. A. Remillard, C. D. Bailyn, M. M. Dwyer, M. Buxton, A New Dynamical Model for the Black Hole Binary LMC X-1, ApJ 697 (2009) 573-591.

[356] A. G. Cantrell, C. D. Bailyn, J. A. Orosz, J. E. McClintock, R. A. Remillard, C. S. Froning, J. Neilsen, D. M. Gelino, L. Gou, The Inclination of the Soft X-Ray Transient A0620-00 and the Mass of its Black Hole, ApJ 710 (2010) 1127-1141.

[357] J. A. Orosz, Inventory of black hole binaries, in: K. van der Hucht, A. Herrero, C. Esteban (Eds.), A Massive Star Odyssey: From Main Sequence to Supernova, volume 212 of IAU Symposium, p. 365. 
[358] J. A. Orosz, J. F. Steiner, J. E. McClintock, M. A. P. Torres, R. A. Remillard, C. D. Bailyn, J. M. Miller, An Improved Dynamical Model for the Microquasar XTE J1550-564, ApJ 730 (2011) 75.

[359] D. J. Hurley, P. J. Callanan, P. Elebert, M. T. Reynolds, The mass of the black hole in GRS 1915+105: new constraints from infrared spectroscopy, MNRAS 430 (2013) 1832-1838.

[360] G. M. Harry, LIGO Scientific Collaboration, Advanced LIGO: the next generation of gravitational wave detectors, Classical and Quantum Gravity 27 (2010) 084006.

[361] T. Accadia, F. Acernese, F. Antonucci, P. Astone, G. Ballardin, F. Barone, M. Barsuglia, A. Basti, T. S. Bauer, M. Bebronne, M. G. Beker, A. Belletoile, et al., Status of the Virgo project, Classical and Quantum Gravity 28 (2011) 114002.

[362] K. Somiya, Detector configuration of KAGRA-the Japanese cryogenic gravitational-wave detector, Classical and Quantum Gravity 29 (2012) 124007.

[363] C. S. Unnikrishnan, IndIGO and Ligo-India Scope and Plans for Gravitational Wave Research and Precision Metrology in India, International Journal of Modern Physics D 22 (2013) 41010.

[364] J. Abadie, B. P. Abbott, R. Abbott, M. Abernathy, T. Accadia, F. Acernese, C. Adams, R. Adhikari, P. Ajith, B. Allen, et al., TOPICAL REVIEW: Predictions for the rates of compact binary coalescences observable by ground-based gravitational-wave detectors, Classical and Quantum Gravity 27 (2010) 173001.

[365] P. C. Peters, Gravitational Radiation and the Motion of Two Point Masses, Physical Review 136 (1964) 1224-1232.

[366] J. Veitch, I. Mandel, B. Aylott, B. Farr, V. Raymond, C. Rodriguez, M. van der Sluys, V. Kalogera, A. Vecchio, Estimating parameters of coalescing compact binaries with proposed advanced detector networks, Phys. Rev. D 85 (2012) 104045.

[367] M. C. Miller, Prompt Mergers of Neutron Stars with Black Holes, ApJL 626 (2005) L41-L44. 
[368] J. A. Faber, T. W. Baumgarte, S. L. Shapiro, K. Taniguchi, F. A. Rasio, Dynamical evolution of black hole-neutron star binaries in general relativity: Simulations of tidal disruption, Phys. Rev. D 73 (2006) 024012 .

[369] M. Shibata, K. Taniguchi, Merger of binary neutron stars to a black hole: Disk mass, short gamma-ray bursts, and quasinormal mode ringing, Phys. Rev. D 73 (2006) 064027.

[370] M. Shibata, K. Uryu, Merger of black hole-neutron star binaries: Nonspinning black hole case, Phys. Rev. D 74 (2006) 121503.

[371] M. Shibata, K. Uryu, Merger of black hole neutron star binaries in full general relativity, Classical and Quantum Gravity 24 (2007) 125.

[372] Z. B. Etienne, J. A. Faber, Y. T. Liu, S. L. Shapiro, K. Taniguchi, T. W. Baumgarte, Fully general relativistic simulations of black holeneutron star mergers, Phys. Rev. D 77 (2008) 084002.

[373] M. Shibata, K. Taniguchi, Merger of black hole and neutron star in general relativity: Tidal disruption, torus mass, and gravitational waves, Phys. Rev. D 77 (2008) 084015.

[374] M. D. Duez, F. Foucart, L. E. Kidder, H. P. Pfeiffer, M. A. Scheel, S. A. Teukolsky, Evolving black hole-neutron star binaries in general relativity using pseudospectral and finite difference methods, Phys. Rev. D 78 (2008) 104015.

[375] Z. B. Etienne, Y. T. Liu, S. L. Shapiro, T. W. Baumgarte, General relativistic simulations of black-hole-neutron-star mergers: Effects of black-hole spin, Phys. Rev. D 79 (2009) 044024.

[376] H.-S. Cho, C.-H. Lee, Analytical Calculation of the Mergers of Black Hole-Neutron Star Binaries, Pub. Astr. Soc. Japan 62 (2010) 315-.

[377] M. Ruffert, H.-T. Janka, Polytropic neutron star - black hole merger simulations with a Paczyński-Wiita potential, A\&A 514 (2010) A66.

[378] M. D. Duez, F. Foucart, L. E. Kidder, C. D. Ott, S. A. Teukolsky, Equation of state effects in black hole-neutron star mergers, Classical and Quantum Gravity 27 (2010) 114106. 
[379] S. Chawla, M. Anderson, M. Besselman, L. Lehner, S. L. Liebling, P. M. Motl, D. Neilsen, Mergers of Magnetized Neutron Stars with Spinning Black Holes: Disruption, Accretion, and Fallback, Physical Review Letters 105 (2010) 111101.

[380] F. Foucart, M. D. Duez, L. E. Kidder, S. A. Teukolsky, Black holeneutron star mergers: Effects of the orientation of the black hole spin, Phys. Rev. D 83 (2011) 024005.

[381] B. C. Stephens, W. E. East, F. Pretorius, Eccentric Black-holeNeutron-star Mergers, ApJL 737 (2011) L5.

[382] M. Shibata, K. Taniguchi, Coalescence of Black Hole-Neutron Star Binaries, Living Reviews in Relativity 14 (2011) 6.

[383] F. Foucart, M. D. Duez, L. E. Kidder, M. A. Scheel, B. Szilagyi, S. A. Teukolsky, Black hole-neutron star mergers for $10 \mathrm{M}_{\odot}$ black holes, Phys. Rev. D 85 (2012) 044015.

[384] Z. B. Etienne, Y. T. Liu, V. Paschalidis, S. L. Shapiro, General relativistic simulations of black-hole-neutron-star mergers: Effects of magnetic fields, Phys. Rev. D 85 (2012) 064029.

[385] W. E. East, F. Pretorius, B. C. Stephens, Eccentric black hole-neutron star mergers: Effects of black hole spin and equation of state, Phys. Rev. D 85 (2012) 124009.

[386] Z. B. Etienne, V. Paschalidis, S. L. Shapiro, General-relativistic simulations of black-hole-neutron-star mergers: Effects of tilted magnetic fields, Phys. Rev. D 86 (2012) 084026.

[387] F. Foucart, Black-hole-neutron-star mergers: Disk mass predictions, Phys. Rev. D 86 (2012) 124007.

[388] V. Paschalidis, Z. B. Etienne, S. L. Shapiro, General-relativistic simulations of binary black hole-neutron stars: Precursor electromagnetic signals, Phys. Rev. D 88 (2013) 021504.

[389] K. Kyutoku, K. Ioka, M. Shibata, Anisotropic mass ejection from black hole-neutron star binaries: Diversity of electromagnetic counterparts, Phys. Rev. D 88 (2013) 041503. 
[390] M. Tanaka, K. Hotokezaka, K. Kyutoku, S. Wanajo, K. Kiuchi, Y. Sekiguchi, M. Shibata, Radioactively Powered Emission from Black Hole-Neutron Star Mergers, ApJ 780 (2014) 31.

[391] T. Futamase, Y. Itoh, The Post-Newtonian Approximation for Relativistic Compact Binaries, Living Reviews in Relativity 10 (2007) 2 .

[392] M. V. van der Sluys, C. Röver, A. Stroeer, V. Raymond, I. Mandel, N. Christensen, V. Kalogera, R. Meyer, A. Vecchio, GravitationalWave Astronomy with Inspiral Signals of Spinning Compact-Object Binaries, ApJL 688 (2008) L61-L64.

[393] M. van der Sluys, I. Mandel, V. Raymond, V. Kalogera, C. Röver, N. Christensen, Parameter estimation for signals from compact binary inspirals injected into LIGO data, Classical and Quantum Gravity 26 (2009) 204010.

[394] V. Raymond, M. V. van der Sluys, I. Mandel, V. Kalogera, C. Röver, N. Christensen, The effects of LIGO detector noise on a 15-dimensional Markov-chain Monte Carlo analysis of gravitational-wave signals, Classical and Quantum Gravity 27 (2010) 114009.

[395] K. Yagi, N. Yunes, I-Love-Q: Unexpected Universal Relations for Neutron Stars and Quark Stars, Science 341 (2013) 365-368.

[396] A. Maselli, V. Cardoso, V. Ferrari, L. Gualtieri, P. Pani, Equation-ofstate-independent relations in neutron stars, Phys. Rev. D 88 (2013) 023007.

[397] J. M. Lattimer, M. Prakash, What a Two Solar Mass Neutron Star Really Means, arXiv:1012.3208 (2010).

[398] R. Narayan, J. E. McClintock, Observational evidence for a correlation between jet power and black hole spin, MNRAS 419 (2012) L69-L73.

[399] D. M. Russell, E. Gallo, R. P. Fender, Observational constraints on the powering mechanism of transient relativistic jets, MNRAS 431 (2013) 405-414. 